\title{
Effectively Employing Young Adult Peer Providers: A Toolkit
}

Jonathan Delman, PhD, JD, MPH

Vanessa Vorhies Klodnick, PhD, LCSW

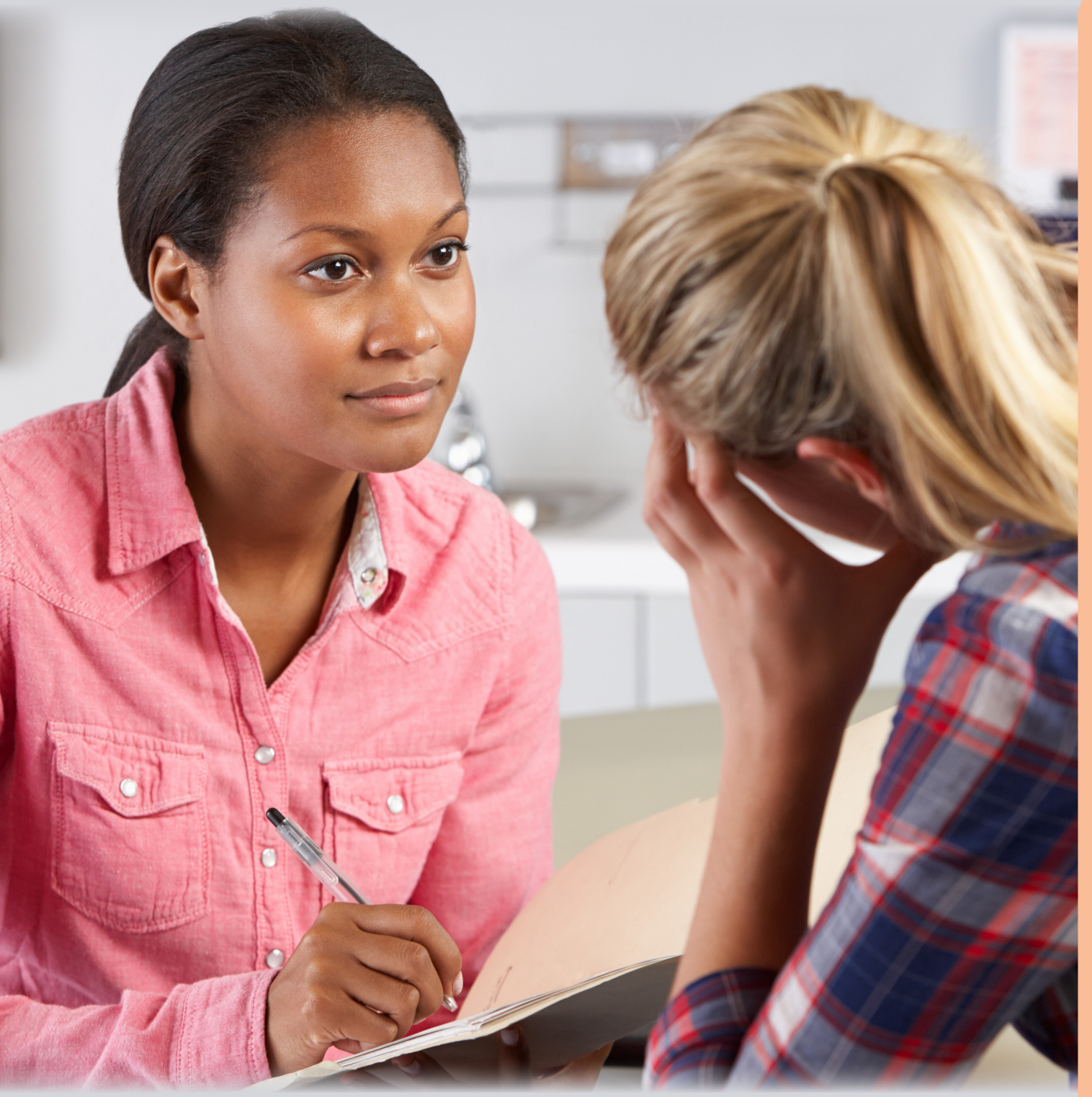

Toolkit for Employers, including:

- Supervising young adult peer providers

- Establishing a supportive organizational culture

- Preparing and engaging nonpeer staff

- Hiring and training young adult peers

- Addressing significant job 


\section{ACKNOWLEDGEMENTS}

\section{Funders}

The contents of this toolkit were developed under a grant with funding from the National Institute on Disability, Independent Living, and Rehabilitation Research (NIDILRR), and from the Center for Mental Health Services of the Substance Abuse and Mental Health Services Administration(SAMHSA), United States Department of Health and Human Services (ACL Grant\# 90RT5031, The Learning and Working Transitions RRTC). NIDILRR is a Center within the Administration for Community Living (ACL), Department of Health and Human Services (HHS). The contents of this toolkit do not necessarily represent the policy of NIDILRR, ACL, HHS, or SAMHSA and you should not assume endorsement by the Federal Government.

\section{Authors}

Jonathan Delman, PhD, JD, MPH, Assistant Research Professor, Transitions Research and Training Center

Vanessa Vorhies Klodnick, PhD, LCSW, Senior Researcher, Thresholds

\section{Editorial contributors}

Rachel Banderob, Executive Assistant, Technical Assistance Collaborative

Sara Burke, BA, Communications Director, Technical Assistance Collaborative

Deirdre Logan, BA, Systems \& Psychosocial Advances Research Center

Susan Doiron, BS, Systems \& Psychosocial Advances Research Center

Gregory Ronco, BA 


\section{TABLE OF CONTENTS}

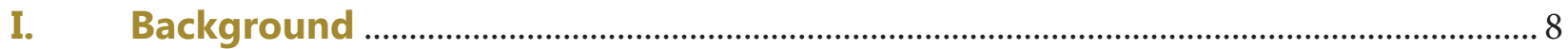

- Young Adults with Serious Mental Health Conditions ....................................................

- Newer Services Designed to Meet the Needs of Young Adults .......................................... 10

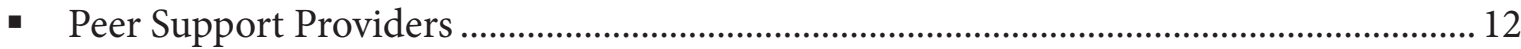

II. Young Adults in the Peer Provider Role................................................................... 16

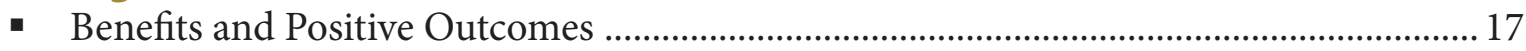

- The Challenges of Implementation ............................................................................... 19

III. Conceptualizing and Structuring the Young Adult Peer Role in Your

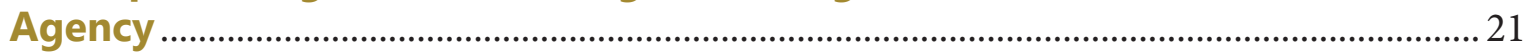

- Conceptualizing and Structuring the Role....................................................................... 22

- Consideration of Relational Boundary Issues ..................................................................... 24

IV. Establishing an Organizational Culture that Supports Young Adult Peers.......27

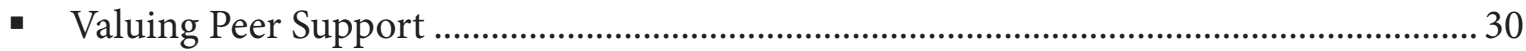

- Understanding the Experiences and Culture of Today's Young Adults............................30

- Recognizing and Addressing Workplace Stigma.................................................................... 33

- Embracing Legal Obligations to not Discriminate Against Employees with

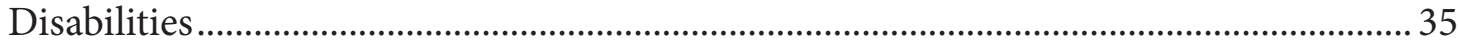

- Diversity of Person, Perspective and Experience …................................................................36

- A Positive Development Approach to Treatment and Services......................................... 38

- Self-determination and Dignity of Risk............................................................................... 39

- Employee Access to Wellness Tools, Services and Supports .............................................. 40

V. Recruiting, Hiring and Training Young Adult Peers................................................ 43

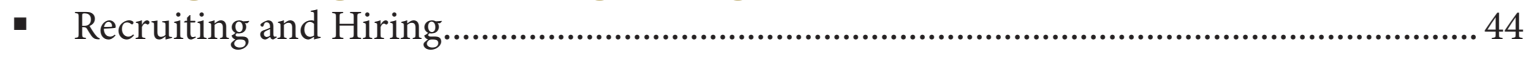

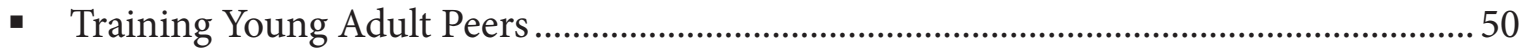

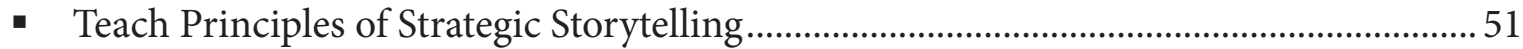

- Review and Discuss Peer Provider Disclosure Policies...................................................... 52

VI. Effective Supervision for Young Adult Peer Providers............................................ 54

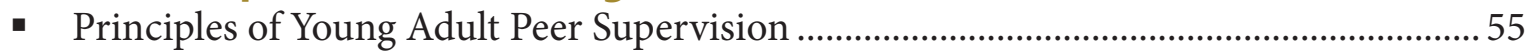

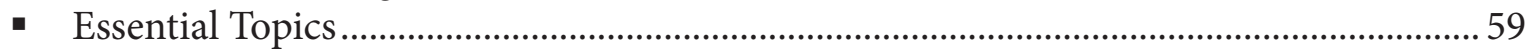

- The Importance of the Young Adult Peer-supervisor Working Alliance ..........................66

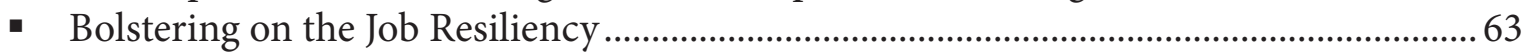

- Training, Supervision, and Support for Young Adult Peer Supervisors .............................64 64

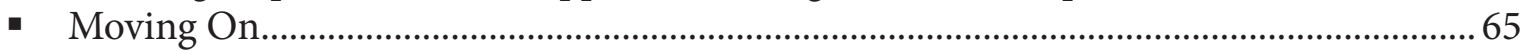


Reasonable Accommodations ......................................................................................66

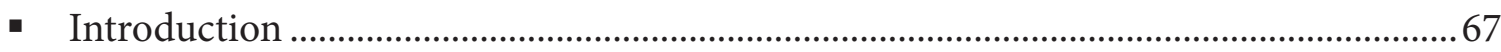

- RA Mandate Basics............................................................................................................ 68

- There are Clearly Described Protocols for Employees to Request Accommodations or Other Assistance for Disability-related Job Difficulties......................................................70

- Employers and Employees Use of Appropriate Interactive Tools and Processes to Discuss RA Solutions ……………………………………………………………...... 71

- RA Responsibilities are Clearly Defined and Understood by the Employer and Staff.77

- Supervisors Have the Willingness and Support to Address Accommodation Issues...78

VIII Preparing and Engaging Non-peer Staff...................................................................79

- Training and Support for Non-Peer Staff ........................................................................... 80

- Team Building Strategies ..................................................................................................... 83

IX. Infrastructure and Framework ............................................................................... 85

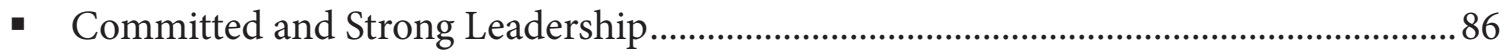

- Strong Organizational Communications That Endorse the Young Adult Peer Role ...88

- Connecting and Working with Human Resources Departments.....................................90

- Effective Staff Hiring and Accountability Practices..........................................................94

- Young Adult Peer Influence on Internal Policies and Practices.......................................95

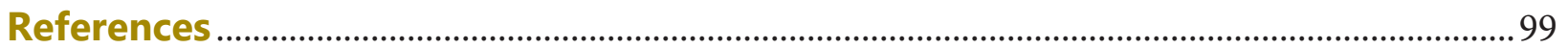

Appendices. 105
A. Organizational Self Assessment
B. Summary of Federal Anti-discrimination Prohibitions
C. Sample YA Job Description
D. Sample Memo Form
E. PDSA Form for Supervision
F. Standards for Assessing Reasonableness and Undue Hardship
G. Leave and Undue Hardship
$\mathrm{H}$. Job Challenges and Accommodations Worksheet 


\section{GLOSSARY/TERMINOLOGY AND ABBREVIATIONS}

FEP - First episode psychosis.

Mental Health Condition (MHC) - a general term referring to conditions that are psychological in origin and result in significant emotional distress (e.g., mental health symptoms), though not necessarily significant functional impairment. These conditions may or may not receive a diagnosis, but are often accompanied by anxiety and/or depression.

NIDILRR - National Institute on Disability, Independent Living, and Rehabilitation Research.

Reasonable Accommodation (RA) - Modifications or adjustments to ordinary business operations that enable a "qualified" individual with a disability to:

- successfully perform essential job functions;

- enjoy equal employment benefits, privileges and opportunities;

- apply for a job.

Reasonable Accommodation Framework - An employer on notice of an employee having disability related job difficulties must engage in an "interactive process" with the employee to reach a resolution on the most appropriate RA(s). The threshold standards to assess whether a proposed accommodation is valid under the circumstances are: 1) Is it reasonable? and, 2) Will the accommodation cause an "undue hardship" (UH) on business operations.

Peer provider ("Peer") - someone who uses his/her lived experience of mental health difficulties to inspire and help clients: (1) to take action towards their recovery and (2) to educate staff on the recovery process and the peer role. Other common terms include "peer specialist", "peer provider", and "peer mentor".

SAMHSA - Substance Abuse and Mental Health Services Administration

Serious Mental health condition (SMHC) - a general term referring to conditions that are psychological in origin and result in significant functional impairment. The term includes but is not limited to diagnoses of schizophrenia, bipolar, major depression, borderline personality disorder (i.e., emotional dysregulation disorder), anxiety disorder, and post traumatic stress disorder (PTSD).

Transitional age youth (TAY) - an older youth or young adult typically between the ages of 16 and 30 .

YA Peer - Young adult peer provider. A YA peer is someone who is near the age to the TAY clients they work with. 


\section{INTRODUCTION}

The University of Massachusetts Medical School, Department of Psychiatry, Systems and Psychosocial Advances Research Center (SPARC), Transitions Research and Training Center (RTC), are pleased to present: A Toolkit for Effectively Employing Young Adult Peer Providers. This toolkit was developed specifically for provider organizations that employ or want to employ young adult peer providers, who we call "YA peers" in this toolkit.

There has been a national call for such a toolkit because:

- The number of young adult peer provider positions is growing exponentially.

- Young adult peer providers on treatment team promote hope, self-esteem and symptom reduction for young adult clients.

- The growth in this field is fueled by positive research evidence and expanding funding opportunities.

- This growth presents new job and career opportunities for young adults diagnosed with serious mental health conditions (SMHC).

- Providers have faced significant challenges in integrating the young adult peer role into clinical and non-clinical services.

Young adult peer providers are uniquely qualified to engage and support young adult clients because of their experience in facing similar challenges and their capacity to display authentic empathy and validation. Peer support is vital for young adults with SMHC, many of whom have lost hope for a fully engaged life. The very presence of YA peers demonstrates to young adult clients that they can be productive and fulfilled members of society. When YA peers are included in treatment teams, young adult clients are more engaged and satisfied with their treatment, and begin to take greater control of their lives. Through peer support, young adult clients learn that their difficult experiences do not lack meaning, but instead instill within them higher levels of empathy and a greater capacity to help others in need.

Along with direct client benefits, the recent expansion of peer positions provides young adults with SMHC a new and unique opportunity to obtain a professional job. By working as a peer provider, these young adults are learning transferable skills, building resumes, developing a social network, and are making progress on their career path.

Despite the growing number of opportunities for young adult peers in provider contexts, providers have struggled to maintain a strong young adult peer workforce. The peer provider position itself is fairly new and unique in relation to other provider staff jobs. Its introduction can be seen by other staff as the provider's lower cost alternative to their jobs, and they can feel threatened, disrespected and concerned about their job status. Often employees are not knowledgeable about the peer role and responsibilities, leading to role confusion and conflict between peer and non-peer staff. It is incredibly challenging for peer providers to be successful without the support of at least some co-workers, as well as individualized, supportive and regular supervision. 
Thus, the provider and program leadership must support peers, co-workers and supervisors to work collaboratively through enhanced trainings, team building strategies, personnel policies, and sharing their enthusiasm for the potential of the YA peer role. This toolkit provides employers with direct guidance on how to enhance their capacity to sustain and grow a young adult peer workforce.

In Chapters 1, 2 and 3, we provide background on the unique experience and needs of young adults with SMHC, the changing landscape of service provision for them, the key features of the young adult peer role, and the benefits and challenges that best embrace the young adult peer role.

Chapter $\mathbf{4}$ discusses the organizational cultural landscape necessary to support young adult peer success, including an organizational self-assessment.

Chapter 5 presents best practices for recruiting, hiring, and training YA peers, with very practical tools and sample approaches.

Chapter 6 offers effective approaches for supervising YA peers toward enhancing job resiliency and functioning and building an organizational culture to support these best practices.

Chapter 7 presents a unique approach for addressing job challenges using the reasonable accommodation framework, with worksheets and exercises.

Chapter 8 focuses on preparing and supporting non-peer staff to be supporters and resources for YA peers.

Chapter 9 discusses the organizational infrastructure necessary for building and maintaining organizational culture and best practices, including leadership, messaging, working with HR (Human Resources), staff accountability standards and ways to strengthen the voice of YA peers in the organization/program. These best practices are designed to improve young adult peer job effectiveness, satisfaction, and longevity, and to reduce absenteeism and turnover.

\section{The toolkit contains:}

- A manual for the organization

- Interactive worksheets and self-assessment forms

- Examples of useful workplace policy approaches and job descriptions

- Webinars on supervision and using the ADA/RA multi-modal framework

Our aim is to integrate key elements within the toolkit that will be beneficial to providers and their staff, though some chapters and other resources will be useful to other stakeholders too. As an initial step, we'd like to share with you 9 takeaways from this toolkit: 


\section{Nine Toolkit Takeaways}

(each followed by the chapter(s) in which they are described)

1. All staff should understand the purpose and unique qualities of the young adult peer role via orientation, training, team building and supervision. $[1,3,8]$

2. Individualized and regular supervision that is reflective and addresses career and skill development, wellness, and relationship development is essential for YA peer job success. [6]

3. Establish YA peer provider job qualifications, functions and pay grade before starting the hiring process, and do so in collaboration with Human Resource (HR). [5]

4. Avoid job qualification exclusions related to criminal history; do not inquire about criminal history on the job application or at the first interview. [5]

5. Orientation and training for YA peers should clarify organizational policies, including those on communications, absences, accommodations, and health benefits. [5]

6. Agency staff must understand the unique developmental experiences of young adults in today's society. [4]

7. When a supervisor is considering an accommodation or job support for a peer, start by identifying the job functions the person is having trouble with before focusing on the person's mental health status and symptoms. [7]

8. Organizational leadership must proactively address stigma and prevent discrimination toward young adult employees diagnosed with mental conditions. $[4,8,9]$

9. Team building activities such as cross training, co-learning and mentoring are important approaches toward YA peers and non-peer staff developing high quality working relationships [8].

This toolkit was developed over a two-year period in consultation with an advisory board composed of young adults diagnosed with SMHC and experts in transition-age youth mental health. It was led and written by two individuals with personal experience in supervising and supporting young adult peers as well as conducting research examining the young adult peer provider role. Using a mixed-methods approach, the authors conducted interviews and focus groups with key stakeholders (e.g., young adult peer providers and supervisors; non-peer and peer administrators; peer provider advocates and experts; and provider human resource departments) and an extensive literature review of peer-reviewed, white, and grey literature pertaining to young adults in the peer provider role. 


\section{1 | BACKGROUND}




\section{1 |BACKGROUND}

\section{Young Adults with Serious Mental Health Conditions}

Transition-age youth (ages 16-30) are at particularly high risk for the development of serious mental health conditions (SMHCs). (We use the term "serious mental health condition" purposefully here because of the lack of a single term that captures both "serious emotional disturbance" and "severe mental illness" for a population that spans child and adult systems.) Seventy-five percent of serious mental health conditions, such as schizophrenia, develop by the age of 24. Each year, roughly $20 \%$ of 18-25 year olds meet criteria for a current mental health diagnosis. ${ }^{i}$ In addition, SMHCs are commonly accompanied by co-morbid health conditions, such as attention-deficit/hyperactivity disorder (ADHD) and substance use disorders.

Historically, young people who have utilized public mental health services as adolescents travel an onerous path into the adult world. Young people diagnosed with a serious mental health condition who age out of the child welfare system are subject to an abrupt end to their mental health services. There is a clear divide between child and adult service systems, each of which has different eligibility criteria and treatment coverage, with the adult systems usually providing less comprehensive coverage and services. Thus, youth who are aging out are likely to lose providers with whom they've developed trusting relationships, and need to find new ones in the adult system. For those who are not in the child welfare system, but are public health insurance recipients (e.g., Medicaid), mental health treatment continuity is often disrupted upon turning 18. In addition, at age 18 young adults can choose whether or not to engage in treatment, and if so whether or not to include their family, which may help or hinder their personal recovery journey.

Many young adults exiting child systems have experienced lengthy stays in residential treatment. Their transition to adulthood is complicated by strained or non-existent relationships with family members and a lack of role models exhibiting maturity and personal responsibility. Many of these young people struggle to maintain an emotionally and instrumentally supportive social network and are at times ostracized from others of their age group. These young adults are at a significant risk for homelessness and criminal justice involvement. Research shows having serious mental health challenges during the transition to adulthood is a strong predictor of health and psychosocial problems in later adulthood. ${ }^{\text {ii }}$

Transition-age youth diagnosed with SMHC usually experience a significant disruption in their vocational development, including delayed high school completion, not enrolling in postsecondary education programs, and not completing such programs when enrolled. While some employment as an adolescent is a significant predictor of employment as a young adult, many adolescents with mental health problems have not worked. One study showed that four years after completing high school only $42 \%$ of young people diagnosed with a SMHC were employed, with 
only $50 \%$ employed 8 years after high school. ${ }^{\text {iii }}$

Adult community mental health services have historically not been effective for, or appealing to, young adults. In addition, adult service providers are often not trained in adolescent development and are thus unprepared for the relatively immature psychosocial development of the transitionage youth (TAY) population. Adult services were designed to rehabilitate functioning rather than teach skills for the first time. Adult-oriented programs typically do not provide preparation for the job search process (e.g., resume development, interview practice), career planning, or postsecondary education, all of which are desired by young adults generally.

Additionally, transition-age youth perceive their needs and circumstances as different from those of older adults, and typically do not want to be in programs with middle-aged and older adults. In comparison to other adults, young adults generally:

- Do not see "recovery" as a process, but instead as a short-term goal, with an emphasis on returning to school or work directly;

- Take much bigger "risks" toward their search to define their identity, in part because the part of the brain that manages impulse control (e.g., the frontal lobe) is still developing;

- Have not developed or are at an early stage of developing resilience and confidence;

- Experience a heightened level of shame, stigma, and the concern that friends will learn about their diagnosis and avoid them. ${ }^{\text {iv }}$

The stigma of being identified as a "mental patient" is another reason young adults do not engage in treatment or ask for other available supports. For example, young adults diagnosed with SMHCs are less likely to inform their post-secondary education program or their employer of their disability and need for accommodations when compared to young adults with other types of disabilities. $^{\mathrm{v}}$

\section{Newer Services Designed to Meet the Needs of Young Adults}

Starting in the 1990s, various stakeholders became acutely aware of the service gaps facing young adults diagnosed with SMHC. Early research efforts, led by Rusty Clark and Maryann Davis, developed and evaluated service models designed to address the expressed needs of young people diagnosed with SMHC. In 2001, Consumer Quality Initiatives (CQI), a peer-run research and evaluation organization in Massachusetts, conducted a participatory action research project co- led by young adults diagnosed with SMHC to identify young adult mental health service needs and gaps. They found that no service system for TAY existed in Massachusetts, and made broad-based recommendations, including the integration of peer mentors in service delivery and an infusion of young adult voice in policy and practice development. In 2008, the United States Government Accountability Office (GAO) followed up with a report on the progress of state and federal governments to address these gaps, finding that young adults continued to have great difficulty finding mental health services appropriate to their age group. With a greater public awareness of these service gaps, parent and transition-age youth-led policy and advocacy organizations (including National Federation of Families for Children's Mental Health and Youth 
M.O.V.E.) began to advocate more widely for the funding of services that meet the specific needs of TAY diagnosed with a SMHC.

In the early 2000s, policy and funding bodies began to encourage mental health service providers to develop programming that was person-centered for adults and family-centered for youth, a movement away from a service structure that provided treatments primarily based on diagnosis and not personal preferences. With the release of the President's New Freedom Commission report in 2003 states began to leverage federal funding to develop policies aimed at transforming the deficit based adult community mental health systems to one that is recovery oriented (i.e., strengths-based, trauma-informed, and responsive to consumer values and preferences). Federal funding also supported states to use Systems of Care principles and Wraparound methods to develop strengths-based youth services tailored to the specific needs of youth and family ("family driven" and "youth guided"). ${ }^{\mathrm{vi}}$

In addition, the U.S. National Institute on Mental Health, through the Recovery After an Initial Schizophrenia (RAISE) initiative, and the Robert Wood Johnson Foundation funded largescale research projects to develop and test service models aimed at improving the outcomes of youth and young adults experiencing a recent onset of psychosis. The results from these research initiatives led to SAMHSA setting-aside 5\% in 2015 and 10\% in 2016 of each state's annual Mental Health Block Grant to be used for state integration of recently identified evidence-based services for first-episode psychosis such as "coordinated specialty care" (CSC). SAMHSA also funded the Healthy Transitions grant program for states and counties to develop a continuum of service strategies for TAY.

As a result of these efforts, new clinical and community based approaches are emerging to meet the needs of young adults diagnosed with SMHC. Core elements across all of such programs are that they:

- Are strengths-based and "optimistic" in approach;

- Focus on a young person's recovery (psychological, social, vocational), not just on mental health symptom control, stability, and functioning;

- Actively involve the clients, and if desired family members, in treatment decision making.

For example, the Transition to Independence Process (TIP) Model is a widely adopted approach that empowers young people to be involved in treatment and goal planning and to choose who he or she wants to be involved in this process. ${ }^{\text {vii }}$ TIP focuses on making progress across five transition domains, including employment and career, education, living situation, personal effectiveness and wellbeing, and community-life functioning. States such as Massachusetts are using SAMHSA funding to develop a Wraparound enhancement grant to develop youth and family services that are appealing to TAY.

Components of Coordinated Specialty Care (CSC) approach has emerged as a best-practice for treating individuals with first episode psychosis (FEP). Major CSC components include collaboration with key stakeholders (e.g., teachers), cognitive and behavioral skills training, low-dosage medications, supported employment/education, case management, family education, and outreach. A recent study found that CSC clients experienced significant improvements in 
symptoms and quality of life compared to those receiving regular care. The peer and vocational elements of these models are in the very early stages of development.

SAMHSA has also worked with the National Institute on Disability, Independent Living, and Rehabilitation Research (NIDILRR) to fund two young adult oriented research and training centers (RTCs):

- The Transitions RTC aims to improve supports for TAY ages 14-30 who are diagnosed with SMHC and trying to successfully complete their schooling and training and move into personally rewarding work lives. TAY involvement in all stages of research and development is a cornerstone of the center. (University of Massachusetts Medical School, Worcester, MA, Department of Psychiatry, Systems and Psychosocial Advances Research Center).

- The Pathways RTC aims to improve the lives of TAY diagnosed with SMHC through the research and development of interventions focused on building young people's assets in four areas: 1) self-determination and positive identity, 2) youth- and young adult-directed decision making, 3) skills needed for adult roles, and 4) supportive relationships with peers and adults. (Portland State University, School of Social Work)

\section{Peer Support Providers}

Peer support providers, or "peers," are individuals in recovery from mental health and/or substance use issues who strategically share their lived experience with clients to inspire hope, provide emotional support, and aid in developing a recovery plan. Peers also encourage and prepare clients to become more involved in their own health and treatment decisions. The validation and bonding over shared-lived experiences between a peer and client is critical in mitigating the power differential that typically exists between professional practitioners and clients.

Peers exemplify that recovery is possible, which is a new notion for many clients who have been led to believe that "stability" and symptom control is their best future. Peers also can share practical skills for navigating the health care system and developing wellness tools. The peer role is often integrated into adult community mental health treatment services. 


\section{Unique Characteristics \& Value of the Peer Role}

Peers are unique in relation to other mental health professionals in four primary ways. They:

- Use their personal recovery story with clients strategically. For example, if a client is having difficulty overcoming a barrier towards recovery, a peer may use an example of how she or he has struggled in similar circumstances but persisted and found resources to overcome such a barrier. A peer may also share community resources they learned about in their recovery journey, such as specific peer groups, websites and communities, and particular providers.

- Are role models/exemplars. Peers are "living proof" that recovery and meaningful employment are possible for people diagnosed with SMHC. In the face of negative stereotypes, peers are concrete examples of recovery, instilling hope in people who may have lost hope.

- Advocate on behalf of the client. Peers advocate for their client's perspective and for greater client involvement in treatment decision making. This includes presenting the client's preferences in staff meetings and case discussions, focusing attention on client interests and strengths, and challenging stereotypes. Peer support staff break down barriers between clients' and providers' perspectives and open channels of communication.

- Engage in Mutuality. When people diagnosed with SMHC talk of mutuality, they speak of a relationship based on respect, compassion, unconditional positive regard, and reciprocity. Establishing mutuality in a paid helping relationship can be a challenge due the inherent power imbalance introduced through professional certification and pay. Thus, peers must focus on their relationship to the client, and not just on client needs. Both parties must recognize that each of them can learn from and be impacted by that relationship, regardless of their specific roles.

In the last decade, the peer role in the U.S. has developed as a profession, accompanied by practice guidelines and codes of ethics. This development has been spearheaded by state and national consumer run organizations, which first introduced the role to providers and state and county governments in the 1980s. State mental health authorities and insurance companies began to take notice and fund such positions. In the late 1990s and 2000s, federal agencies such as SAMHSA, the Centers for Medicare \& Medicaid Services (CMS), and the Veterans Administration (VA) developed policies and funding sources to encourage providers to incorporate the peer role into services. These developments have generated exponential growth in the peer position. Based on information provided by 31 states, the number of peer positions is estimated at 13,000 nationally. In the VA alone there are at least 1,000 peer positions. ${ }^{\text {viii }}$ Further growth has been fueled by the Affordable Care Act's (ACA) behavioral health parity requirement, which, along with a shortage of traditional providers, has elevated the peer role as a federal and state funding priority. With regard to young adult peer positions specifically, federal and state agencies have begun to create policies to support that role in child/adolescent and adult mental health services. ${ }^{\mathrm{ix}, \mathrm{x}}$ For example, in 2015, CMS and SAMHSA issued an informational bulletin to states offering guidance on designing Medicaid 
benefit packages that include peer support services for young adults experiencing a recent onset of psychosis. ${ }^{\mathrm{xi}}$ Some states have used SAMHSA's systems of care enhancement grants to embed young adult peers in Medicaid funded wraparound services.

While lived experience of mental illness and recovery is necessary for the peer role, training and certification as a "Certified Peer Specialist" (CPS) is an important credential which many providers and state governments now require or favor for peer employment. ${ }^{\text {xi }}$
The peer role provides TAY, particularly those with limited or no post-secondary education and/or employment experiences, with a new opportunity to enter the labor force, obtain a professional job, learn transferable skills, build resumes, and network towards a self-desired career path

Certification requires that peers be trained in the basic set of core competencies (see unique characteristics above and code of ethics below), and then pass an exam demonstrating those competencies. CPS trainings are offered in most states, though training requirements and certification standards vary by state and organization. The required number of training hours ranges from 30 to over 100, the required amount of work or volunteer experience ranges from none to approximately 500 hours, and the training curricula highly varies. ${ }^{\text {xiii }}$

\section{Peer Specialist Code of Ethics- Massachusetts}

1. The primary responsibility of Certified Peer Specialists is to help people achieve what they want most in life, their own goals, needs and wants. Certified Peer Specialists will be guided by the principles of self-determination for all.

2. Certified Peer Specialists will maintain high standards of personal conduct. Certified Peer Specialists will also conduct themselves in a manner that fosters their own recovery and integrity.

3. Certified Peer Specialists will openly share their recovery stories, and will likewise be able to identify and describe the supports that promote their recovery.

4. Certified Peer Specialists will, at all times, respect the rights and dignity of the people with whom they work.

5. Certified Peer Specialists will never intimidate, threaten, harass, use undue influence, physical force, or verbal abuse, or make unwarranted promises of benefits to the individuals with whom they work.

6. Certified Peer Specialists recognize that everyone is different and we all have something to learn from one another. Therefore, Certified Peer Specialists will not practice, condone, facilitate or collaborate in any form of discrimination on the basis of ethnicity, race, sex, sexual orientation, age, religion, national origin, marital status, political belief, mental or physical disability, or any other preference or personal characteristic, condition or state. 
7. Certified Peer Specialists will advocate as a partner with those they support that they may make their own decisions in all matters when dealing with other professionals.

8. Certified Peer Specialists will respect the privacy and confidentiality of those they support.

9. Certified Peer Specialists will advocate for the full integration of individuals into the communities of their choice and will promote the inherent value of these individuals to those communities. Certified Peer Specialists will be directed by the knowledge that all people have the right to live in the least restrictive and least intrusive environment of their choice.

10. Certified Peer Specialists will not enter into dual relationships or commitments that conflict with the interests of those they support.

11. Certified Peer Specialists will never engage in sexual/intimate activities with those to whom they are currently providing support, or have worked with in a professional role in the past year.

12. Certified Peer Specialists will keep current with emerging knowledge relevant to recovery, and openly share this knowledge with the people with whom they work.

13. Certified Peer Specialists will not engage in business, extend or receive loans, or accept gifts of significant value from those they support.

14. Certified Peer Specialists will not offer support to another when under the influence of alcohol or when impaired by any substance, whether or not it is prescribed. 


\section{2 | Young Adults in the Peer Provider Role}




\section{2|YOUNG ADULTS IN THE PEER PROVIDER ROLE}

\section{Benefits and Positive Outcomes}

"I was going to this program for a few months and I wasn't getting anywhere. I was feeling better in a way... the medications helped... but I was just there... saw my psychiatrist and attended a few groups, but it was like I was frozen in time. Then one day, the psychologist I was seeing said that she wanted to introduce me to someone who had been there and could give me the help I didn't think I was getting called her a peer specialist. Oh my god, this person had pretty much been where I was. She was working full time, and told me I was not alone. She brought me to groups and introduced me to other people trying to get out of this. I was so much more hopeful, and she started talking about me going back to school, and she helped me with that, and I'm there. But would not be here without the peer specialists"

Implementation of the young adult peer role can have a multi-dimensional set of benefits for society, as summarized in the table below. We have previously discussed how the unique properties of peer support can educate, inspire, and strengthen the client to take steps towards his/her recovery. In that spirit, it is clear that young adult clients benefit greatly from engaging with near age peers who are further along in their recovery. Young adult peers can share similar and recent experiences of vocational and social disruption. The sense of mutuality, closeness, and trust between peer and client directly generates hope and confidence. Young adult clients begin to see that their painful experiences have meaning, enhancing their capacity to be empathetic and help others. A YA peer's very presence within an agency or program setting demonstrates to clients that they can re-engage in desired vocational and social pursuits in a relatively short period of time and without waiting for "full recovery." The table below summarizes the scope of these positive impacts:

\section{Positive Impacts of Introducing YA Peer Role}

\begin{tabular}{|c|c|}
\hline Beneficiary & Positive Impacts \\
\hline Young Adult Clients & $\begin{array}{l}\text { - } \text { Hope } \\
\text { - } \text { Empowerment } \\
\text { - Self-esteem } \\
\text { - Symptom reduction }\end{array}$ \\
\hline
\end{tabular}




\begin{tabular}{|c|c|}
\hline Non-Peer Staff & $\begin{array}{l}\text { - Learn about recovery potential for clients } \\
\text { - Stigma reduction } \\
\text { - Increased insight into the lived experience }\end{array}$ \\
\hline Young Adults with SMHC & $\begin{array}{l}\text { - Employment opportunities } \\
\text { - } \quad \text { A foundation for future career pursuits }\end{array}$ \\
\hline Service Systems & $\begin{array}{l}\text { - Engagement } \\
\text { - } \text { Effectiveness } \\
\text { - } \text { Cost-savings } \\
\end{array}$ \\
\hline
\end{tabular}

The multidimensional impacts of peers within an agency that works with TAY diagnosed with SMHC include:

Client outcomes: Research shows that clients benefit in multiple ways from working with YA peers, from improvements in hope, self-determination, and mental health to improvements in postsecondary educational attainment when that is the focus of the work. ${ }^{\text {xiv }}$ These benefits are likely to be stronger when the peer is working from an established curriculum, helping the client to set and achieve specific personal goals. ${ }^{\mathrm{xv}}$

Providers: Young adult peers strengthen clinical team performance by educating team members on the recovery process and by advocating for the inclusion of client preferences in treatment planning. Just by doing their job with integrity, YA peers will likely force staff to confront some stereotypical views they have of clients. In addition, YA peers, with their recent experience of transition-age mental health problems, are important engagement resources for young adults with SMHC. ${ }^{\text {xvi }}$ A recent study showed that giving a young adult client access to a YA "advocate" positively correlated with service satisfaction and participation in treatment planning. ${ }^{\text {xvii }}$

Young adults diagnosed with SMHC often struggle to find and hold jobs. Like their age-related peers, young adults diagnosed with SMHC perceive employment as an opportunity to become a valued and independent member of society. The expanding peer role also likely has a positive impact on the vocational and career development of YA peer providers. The peer role provides these young adults with a new and unique opportunity to obtain a professional job. By working as a peer, these young adults are learning transferable skills, building resumes, developing a social network and making progress on their career path.

Service system: Client engagement is a major barrier to service use for young adults diagnosed with SMHC. Recent evidence shows that when a YA peer is part of a treatment team, young adults will be more likely to try treatment services, and engage with staff. ${ }^{\text {xviii }}$ In addition, effective YA peer services can result in cost savings, particularly by reducing the use of hospitals and emergency rooms. 


\section{The Challenges of Implementation xix}

- Job inexperience. Since the peer job often is the first professional position for a young person diagnosed with a SMHC, she or he may not yet understand the responsibilities of "work" and have difficulty adapting to workplace norms, particularly with regard to inter-office communications and attire.

- YA peers are also in recovery, and are likely to be contending with their own mental health struggles while concurrently providing support to young people facing a similar transition to adulthood.

- The YA peer role itself can also be challenging. For example, in practicing mutuality, YA peers must learn to balance respect for clients' privacy with their duty to inform the provider and necessary entities when a client displays evidence of danger to self or others - or engages in high-risk behaviors that are negatively impacting engagement and/or progress towards goals.

- Provider workplace culture often presents serious challenges to young adult peer job success because their very presence on treatment teams challenges existing clinical paradigms. Most providers value a clinical approach involving professional expertise, relational boundary maintenance, and risk averseness, while peer support relies on a culture of informality, disclosure, and collaborative problem-solving. Historically accepted clinical models view the sharing of practitioner personal stories with clients as taboo and harmful - driven by the belief that clients come to professionals to share their struggles not the reverse.

- Peer job clarity and confusion. Provider staff often lack clarity on the specific duties and expectations of the peer role. Thus they have concerns about the introduction of the peer role's impact on their own job, both in the treatment process and in employment hierarchy. This difficulty stems in large part from the peer role being poorly defined and staff not being educated on working with people/peers, who bring a different perspective to service provision. This role confusion can lead to conflict with peers on many levels, from treatment decisions to office policies. With this lack of clarity, team leaders may expect peers to carry out tasks contrary to peer role ethics, such as distributing medications and being a representative payee (responsible for managing the client's supplemental Social Security income). The result is tension between peer and not-peer staff.

- New non-peer staff job responsibilities. It is generally expected that treatment staff are to work as a team, and thus improve outcomes and productivity. Thus, while the YA peer will likely reduce the workload some staff, staff will need to take on new responsibilities, including:

- Connecting clients to YA peers providers;

- Coordinating services in collaboration with YA peers,

- Supporting the inclusion of YA peer voice in team meetings.

Provider organizations however do not make this explicit for their staff, adding to job confusion and conflict. 
- Stigma. In addition, recent research shows that at least some provider employees discriminate against people diagnosed with SMHC and attribute stereotypes to diagnostic labels. Among the most common stereotypes are that people diagnosed with mental health conditions lack the necessary knowledge, skills, and abilities for employment, and that they are more costly to employ. At times, there can also be resentment towards peer providers, who non-peer staff may perceive as threats to their jobs..$^{\mathrm{xx}}$ It is not uncommon for YA peers to report that their workplace is unsupportive of their work and their success.

On the other hand, we do know that some providers have effectively implemented the young adult peer role in a supportive and results oriented way.xxii The fact is that young adult near-age peers greatly value their job and perceive it as an opportunity to use their lived experience to motivate and assist other young adults diagnosed with SMHC.

The next chapters of the toolkit provide employers with information and tools to leverage young adult peer enthusiasm and resourcefulness toward minimizing job implementation barriers and establishing the young peer role as a key adjunct to effectively engaging and supporting young adults in reaching their goals. 


\section{3 | Conceptualizing $\boldsymbol{\&}$ StRUcturing the Young Adult Peer Role in Your Agency}




\section{3| CONCEPTUALIZING \& \\ STRUCTURING THE YOUNG ADULT PEER ROLE IN YOUR AGENCY}

\section{Conceptualizing AND STRUCtURING THE ROLE}

It is essential that your agency know what you want "peer support" to look like in your context and that you review what peer support looks like as it rolls out at your agency. In the beginning, a steering committee should meet at least monthly to discuss issues that arise with the integration of YA peers. After a year, this meeting can happen quarterly.

Consider sustainability early on. What kinds of funding do you have or need in order to support this role? Some states fund individuals with Certified Peer Support credentialing - will your agency expect young adults to have this when hired - or can they earn credentials while employed as a YA peer?

Establish key elements of the YA peer role in your context first. These do not need to be set in stone, but they need to be considered in order for YA peers to be successful:

- How many peers does your agency aim to integrate? (At least two YA peers are important for infusing YA peer perspectives at your agency.)

- Consider the valuable kinds of life experiences (past and present) that YA peers should have in your context in order to fulfill the "peer" role.

- What other commitments, especially work, school, volunteer, caretaking responsibilities, will be of value in the YA peer role? What treatment and life experiences, types of mental health conditions, and system involvement (past and present) are of value for those in the YA peer role?

- How many hours will YA peers work each week? Will this be uniform across YA peers or negotiable on an individual basis?

- Who will supervise the YA peers, administratively and/or clinically? You want to identify a staff member who enjoys working with young adults and is motivated to champion the integration of the YA peer role.

- Develop social media \& relationship policies (see chapter 9). Establish if the client YA peer/ client relationship can or should last beyond the professional connection established at your agency.

Consider how your agency will match young adult peer providers to young adult clients. Integrate choice and flexibility as much as possible in the client-YA peer matching process. Clients should have the opportunity to choose which YA peer they want to work with. (This is why it is important to have more than one YA peer at your agency.) Consider how YA client - YA-peer relationships can develop more "naturally" though agency group activities or events. Recognize 
that not every young person will enjoy and/or benefit from peer support. Develop a clear protocol for how YA clients are referred to and matched to YA peers. If a client is not interested in YA peer services at enrollment, providers should suggest connecting with a YA peer at key times when the client needs multiple perspectives to make a decision or when demonstrating anxiety about the future and their recovery.

Discuss how much of the time YA peer-client interactions should be spent specifically on client goals that are part of an established treatment plan. For example, in our project developing YA peer employment specialists, we suggested that between $25-40 \%$ of meetings should be spent on vocational topics because the goals of the interaction were to support engagement in work and/or school. It is important to recognize that sometimes less time will be spent on achieving treatment goals during meetings, and that is okay. New YA peers may be frustrated with not being able to keep their YA clients "focused" on the tasks at hand. Remind YA peers that engagement is a journey and that their interactions with clients take place on a continually shifting continuum of disclosure, validation, and understanding. In theory, the bond that forms through the communication process between a YA peer and YA client is important for achieving outcomes. Thus, focusing more on the "bond" or "alliance" than on the outcome may be a helpful approach for YA peers who do not see the change in their clients that they desire/believe is possible.

Determine where peer support happens. Will YA peers work on-site and/or off-site? What percentage of the time will be on-site and off-site? Oftentimes, young people do not want to meet in a mental health clinic setting, but rather in the community. Ensure that YA peers are able to travel to meet YA clients and if possible, set aside money in the program budget for peer support to happen over a cup of coffee at a local coffee shop. Community integration is key for young people. It opens up the possibility of risk (e.g., risk of confidentiality loss), but it will increase the likelihood that the YA peer and YA client connect and continue connecting.

Determine how much peer support mimics other team member activities with the YA client. Peer support and other team member activities (e.g., therapy and case management) should complement, not undermine each other. Communication among team members is necessary in order to ensure that the YA peer role is differentiated from other non-peer staff roles. Billing codes will often dictate what types of activities each role will have with a YA client. Thus, sometimes YA peers may be responsible for activities that non-peer professionals are not able to bill for. As such, it's helpful to simply be aware of what each member on the team is responsible for. Clarifying the peer role is a process at each individual agency.

Minimize YA peer role confusion. Confusion about the YA peer role within an agency can create on-the-job stigma towards the YA peer. The young adult peer role takes on different forms depending on the agency's service design and culture, the needs and characteristics of young adult clients, and the experience and approach of the young adult peer. Most often, young adult peers take on the role of supporter, mentor, advocate, service engager, navigator, and service provider. Confusion about the young adult peer role can exist on multiple levels, including (but not limited to): 
- YA peers being confused about their role and day-to-day job duties.

- YA peers supervisors being confused about their role as a YA peer supervisor as well as the YA peers role at the agency.

- Other staff, especially non-peer staff, being confused about the YA peer role and what their interactions with YA peers should look like. From an administrative level, the role of the YA peer appears clear: that a young person will provide support to young adult clients to enhance their recovery and personal goal attainment. But how the role is established at your agency is where it can become confusing. In order to avoid YA peer role confusion:

o Define specific responsibilities of the peer role and refine these responsibilities systematically using a steering committee with varying stakeholders (e.g., including YA peers, their supervisors, human resource specialists, and non-peer staff ).

o Write a clear YA peer job description (see chapter 5).

o Have a kickoff where the YA peer role is explained clearly.

o Incorporate a description of the YA peer role and how the role enhances agency culture and client outcomes in the agency's new employee orientation.

\title{
Consideration of Relational Boundary Issues
}

\author{
"She understands where I am coming from and I understand where she is coming from." \\ (Participant in Vocational Peer Mentor Study at Thresholds ${ }^{\mathrm{xxi}}$ )
}

To some extent mutuality must exist for peer support to work. Peer support is based on the idea that if both the client and peer provider are authentic in revealing, discussing, and reacting to each other's lived experiences, there will be an immense benefit for both parties involved. Based on empirical data, Spencer and colleagues (2006) ${ }^{\text {xxii }}$ theorize that collaboration, empathy, authenticity, and (perhaps scariest for non-peer providers) "companionship," are key relational processes in peer support transactions. In this regard, providers must recognize that the "client" role will sometimes shift closer to the "helper" role. It feels good to help. And, part of the healing process in many cultures and treatment philosophies is the act of giving and helping.

When providers are considering employing young adult peers, their greatest concern is the assumed risk of young people's inability to manage mutual relationships and "relational boundaries."

Having this fear is not necessarily negative (as it is important to explore fears associated with any new practice that aims to improve the lives of our clients). Rather, it's asking "why" does it make our toes curl to think about young adults with lived mental health experiences provide support to other young adults? As an agency, acknowledge this fear by creating opportunities for staff to process their perspectives. Teach staff about the unique features of YA peer-client relationships, which include: collaboration, empathy, authenticity, trust, and companionship, ${ }^{\text {xxii }}$ as well as mutuality. Even with mutuality in YA peer-client relationships, YA peers can learn to and establish clear relational boundaries.

It is very important to understand that in all peer support, but perhaps more so with young adult 
peer support, the extent to which the relational boundaries are clarified. Within your agency, draft policies about the key aspects of healthy relationships for all staff to adhere to. For YA peers, ensure that these policies are introduced during orientation, and that the YA peer supervisor and new YA peers process them together. Ideally, after reviewing these policies, the YA peer should sign a commitment letter acknowledging relationship guidelines. These should be reviewed systematically in supervision - at least quarterly.

\section{General Guidelines:}

1. Limits to mutuality must be clearly defined in agency policies and practice. For instance, prohibiting sexual relationships between young adult peers and clients should be established early on. However, it should be recognized that it is not abnormal if a young adult peer has romantic thoughts about or is physically aroused by a client, and it is something a young adult peer should not be ashamed of. Rather, it is an important topic to discuss with one's supervisor in order to put safeguards in place.

2. Managing young adult peer provider-client relational boundaries gets tricky in small towns and neighborhoods as peer provider and client social networks likely will overlap. Young adult peer providers (with the support of their supervisor) must discuss the potential for community encounters and inquire with the client as to how they prefer to describe their relationship. Supervisors should encourage young adult peer providers to tell them when they run into clients in the community during off-hours so that this remains a regular topic during supervision. By doing so, peer providers and supervisors can continually address the somewhat awkward and confusing moments where neither the client nor the peer mentor wanted to be outed as to their affiliation with a mental health organization.

3. Establishing boundaries and rules around the online relationship of young adult peer providers and clients is necessary. Messaging on social media websites like Facebook and Instagram are a primary mode of young adult communication. Each agency will have a different policy on social media interactions and it is important that these policies are reviewed with young adult peer providers and young adult clients. If there are no policies for online communication, then, as a team, discuss and document rules for online communication. For young adults, transparency is key.

Real Life Example. Managing Relational Boundaries. Tania was a bubbly, new, and very motivated vocational peer mentor who reported that at a family reunion on a Sunday afternoon, she was on the phone with a client for over 2 hours. She reported this proudly, "I really got through to her. She was a mess." I carefully probed around where she took the call, why she took it, what it demonstrated to her client, and how she as the peer personally felt during and after the call. We brainstormed as a group how to respond to this in the future in order to support healthy communication and preserve personal wellness. However, Tania felt important to get a call on the weekend - that someone needed her immediate help. And, by taking the call, it allowed her family to observe her working, which was personally meaningful to Tania. It is necessary to process and understand why communication is happening at times between clients and peers that might make a traditional mental health practitioner uncomfortable - and then to explore what makes the most sense for supporting a healthy working alliance between peers and their clients. 
Texting is a primary communication method for young adults. In our experience, texting is a major engagement tool for young adult peer providers. However, this can be taxing on a young adult peer provider if a best practice for texting is not established early on in the YA peer-client relationship. Texting practices will vary from agency to agency. We suggest developing a simple document to guide conversation about texting etiquette in your setting. Also, if young adult peer providers are texting and calling their clients, their cell phone bills should be in part covered by the agency. Depending on a cell phone plan, it may be difficult to determine how many minutes/ data is used for work. In that case, it may make sense for YA peers to have a separate cell phone for work. 


\section{4 | Establishing AN Organizational Culture that Supports Young Adult Peers}




\section{4|ESTABLISHING AN ORGANIZATIONAL CULTURE THAT SUPPORTS YOUNG ADULT PEERS}

Organizational culture is a system of shared values, beliefs, and norms that governs how people behave in organizations. Many studies demonstrate the strong impact of the culture of a mental health provider on day to day staff practices ${ }^{\text {xxiv }}$ Mental health provider culture is a deep reflection of historically ingrained practice norms and well-established professional codes of ethics, which is inexorably linked to the ways in which staff approaches care. Therefore, introduction of innovation and new practice often has to be accompanied by changes in organizational culture. ${ }^{\mathrm{xx}}$ This is particularly true for introducing the role of the young adult peer, which can challenge or contradict typical treatment team norms.

The cultural attributes (e.g., values, beliefs, norms) necessary for effective implementation of the young adult peer role are listed in the table below, which also notes the toolkit chapters outside of this one that provide additional descriptive information. These attributes have been drawn from practice supports described in other chapters in this toolkit, best practices, stakeholders guidance, and the research literature on YA peer success.

\begin{tabular}{|c|c|c|c|}
\hline Beliefs/Norms & $\begin{array}{l}\text { Source(s) \& } \\
\text { Descriptive } \\
\text { Information }\end{array}$ & Values & $\begin{array}{r}\text { Source(s) \& } \\
\text { Descriptive } \\
\text { Information }\end{array}$ \\
\hline $\begin{array}{l}\text { Staff understands and } \\
\text { supports the YA peer role } \\
\text { ( \& culture) }\end{array}$ & Chapters 1-3 & $\begin{array}{l}\text { Respect for young adult } \\
\text { life/culture }\end{array}$ & Chapter 8 \\
\hline $\begin{array}{l}\text { Stigma and Discrimination } \\
\text { are actively addressed at all } \\
\text { levels }\end{array}$ & Chapters 8,9 & $\begin{array}{l}\text { Diversity of people, } \\
\text { perspectives, and } \\
\text { experiences }\end{array}$ & Chapter 8 \\
\hline $\begin{array}{l}\text { Clinical care is strengths- } \\
\text { based and encourages client } \\
\text { empowerment and self- } \\
\text { determination }\end{array}$ & Chapter 8 & $\begin{array}{l}\text { Flexibility (toward } \\
\text { policies, employees and } \\
\text { clients) }\end{array}$ & Chapter 6 \\
\hline $\begin{array}{l}\text { Employee job success is } \\
\text { actively supported }\end{array}$ & Chapters 5-7 & Employee wellness & Chapter 6,8 \\
\hline
\end{tabular}


"Organizational elements" are the normative expectations of staff and the resources that exist to support a desired culture within an organization.

\section{Organizational Elements vital to young adult peer success}

1. Valuing peer support

2. Understanding the experience and culture of today's young adults

3. Recognizing and addressing workplace stigma

4. Embracing legal obligations to not discriminate against employees with disabilities

5. Diversity of person, perspective, and experience

6. A positive [young adult] development approach to treatment and services

7. Self-determination and dignity of risk

8. Employee access to wellness tools, supports and services

As you read through these elements, think about how your agency is structured, specifically the kinds of policies and practices that are inclusive of and exclusive to young adult peer culture. For example, we find that some non-peer staff members express concern about the peer role based on a single negative experience with someone in a peer role. Consider how you might address related staff misconceptions. We also find that when employees do not feel valued, they are more likely to focus on the negatives of their job and their clients, leading to an even more challenging work environment for YA peers. As we are in the early stages in developing both the YA peer role and effective transition-age youth services, not all of these elements are typically in place for providers. Thus, in chapters 7 and 8 we discuss the organization's structures and systems for introducing and maintaining these important cultural elements.

To help you and your agency consider the extent to which your agency policies and practices embrace young adults and peer culture, we have provided self-assessments for the organization overall (Appendix A) and for staff, the latter developed by California Association for Social Rehabilitation Agencies (CASRA), in their guide, Meaningful Roles for Peer Providers in Integrated Healthcare (page 98). 


\section{Valuing Peer Support}

Mead (2003) defines peer support as "a system of giving and receiving help founded on key principles of respect, shared responsibility, and mutual agreement of what is helpful."xxi In many communities, organizations or individuals sponsor peer support groups for people with serious mental health conditions, in which participants share their experiences and perspectives and offer understanding. For newcomers, peer support groups are welcoming and often enlightening. A person who has been struggling with a mental health challenge often feels very discouraged and alone; when he/she learns that there are other people like him/her who are further along in their recovery, the result is often relational connection and hope.

As it was introduced in the context of health care, the formalized peer provider role was developed to bring a peer support perspective to treatment and services. Many people diagnosed with SMHC become peer providers because they themselves have experienced the benefits of peer support. They feel a calling to inspire others by sharing their experiences and knowledge with those who are experiencing significant mental health difficulties.

Thus it is important for non-peer staff to appreciate and understand the peer support cultural norms peers will be drawing from in their work. Many of these are directly connected cultural attributes necessary to support the work of YA peers:

\begin{tabular}{|c|c|c|c|}
\hline \multicolumn{4}{|c|}{ Peer Support Cultural Norms } \\
\hline \multicolumn{2}{|c|}{$\begin{array}{l}\text { Promoting social networks of } \\
\text { support }\end{array}$} & Strengths-based & Non-hierarchical \\
\hline $\begin{array}{l}\text { Partnering } \\
\text { (e.g., } \\
\text { providers, } \\
\text { family) }\end{array}$ & $\begin{array}{l}\text { Self- } \\
\text { determination }\end{array}$ & Flexible & Non-judgmental \\
\hline
\end{tabular}

Some useful tools for assessing the provider's level of peer support culture can be found in the Commonwealth of Pennsylvaniass Tools for Transformation Series: Peer Culture/Peer Support/ Peer Leadership:

- Peer Leadership Provider Checklist

Pages 12-15

- Person in Recovery Working with Provider Checklist

Pages $16-19$

- Staff Checklist

Pages $22-24$

\section{Understanding the Current Experiences and Culture of Today's Young Adults}

The "transition to adulthood" is a complex and stressful time period, distinct from other 
developmental stages. The transition to adulthood comprises the end of childhood and beginning of adulthood - from approximately ages 16 to 30. It is not quite full adulthood, which has historically been seen as one's willingness to accept responsibility for one's actions and the achievement of financial independence. Today, given the difficulty today many adults have in achieving "financial independence," full adulthood is now seen more loosely as some combination of stable adult work, family responsibility, and community involvement. ${ }^{\text {xxvii }}$

The transition to adulthood is a time period in which young people take on increased responsibilities across life domains with decreasing levels of support. It is also a time of heightened uncertainty, as one takes steps in developing their identity by trying out new jobs, school programs, relationships, and living situations - all while renegotiating their relationships with their parents or caregivers and learning how to live on their own.

People with SMCH transitioning to adulthood struggle with forming their identity and taking on responsibilities, often complicated by challenging relationships with their parent(s) and other family members. As TAY improve with treatment, families may not be ready for them to take on new responsibilities for independent living and decision-making. Learning responsibility and forming an identity is a trial and error process for transition- age youth, with or without mental health challenges - and it is through hardship and mistakes that we learn a lot about who we are.

Middle-aged and older adults often struggle to relate to or understand current young adult perspectives by simply extrapolating from their own young adult experiences. Young adults today share a time in history unique to them, which influences how they think and act.

The current group of young adults has grown up with 9/11 and the "great recession," with greater security measures and a less predictable economy. Because of the major recession and a more global economy, young adults frequently find themselves with a significant amount of college debt, while unable to find a well-paying job; this causes young adults to rely on family for financial and housing support for a longer period of time than previous generations. Below are five key aspects of young adult life that organizations should be aware of when employing YA peers.

\section{Communication with technology \& social media}

While people of all ages are likely to possess some form of technology for communication, young adults are typically more tech-savvy, navigating social media and smart phone applications with increased ease compared to older adults. ${ }^{\text {xviii }}$ Today's youth and young adults gravitate away from email and phone calls, choosing instead to communicate through texting, social media, and chat and messaging applications, including video conferencing such as Skype. Young adults adopt technology rapidly because they want to connect to different kinds of people locally and across the globe. For instance, young people welcome "followers" on Instagram, where they can "like" similar images, learn from one another, and communicate via the messaging application within Instagram. This experience can be a form of peer support and potentially a powerful tool that young adult peers might use to engage their young adult clients. However, high levels of internet use for young adults have been associated with increased feelings of insecurity and isolation. ${ }^{\text {xxix }}$ 


\section{Substance use}

Substance use in late adolescence and early adulthood is the norm rather than the exception. There are three major trends affecting young adult behaviors in this area: growing abuse of opioids/heroin (a major cause of death), increasing rates of binge drinking, and the increasing legalization of marijuana (known to have greater potency than in the past). ${ }^{\mathrm{xx}, \mathrm{xxxi}}$ This current generation of young adults show higher usage rates of alcohol, marijuana, and opioids than previous young adult cohorts. ${ }^{\text {xxii }}$ In addition, compared to other age cohorts, young adults comprise the largest group of abusers of Attention-Deficit Hyperactivity Disorder (ADHD) stimulants and prescription opioid pain relievers.

Many peer providers are in recovery from co-morbid SMHC and substance use, and they utilize that knowledge and experience to provide support to their clients. Employers should have policies and structures in place to support the continued employment of a YA peer provider whose job performance drops in relation to known substance use. This can be done using the wellness approaches discussed in this chapter and, often through the provision of reasonable accommodations (see chapter 7).

\section{Economic, employment, and career uncertainty}

Today, underemployment and financial struggles are common for individuals in their 20's because well paid entry-level jobs are hard to find. Health insurance and other non-salaried benefits are becoming increasingly rare, which is why the Affordable Care Act (ACA) included a clause stating that young adults can continue coverage under their parents' health insurance until age 26. While advanced education continues to be vital to earning higher wages and obtaining increased job security, college costs have risen considerably. Many students struggle to finance these costs and rely on student loans, only to find themselves in debt, unable to afford housing, and relying extensively on their family for financial and housing stability.

\section{Family formation}

Young adults today are taking more time to form their own families, including finding a permanent life partner and/or becoming parents. In 1970, the median age of first marriage for males was 24 and for females was 22; in comparison, in 2010 the median age of first marriage for males was 29 and for females was 27. And while many young adults, regardless of social class, endorse the legitimacy of marriage and parenthood, it appears that young people from lower socioeconomic backgrounds are less likely to marry. Such young adults are more likely to have children outside of marriage before being able to support them. These pregnancies tend to be unplanned, with few biological parents prepared to support a family. Without consistent support, they rely on their own families for economic and social support. Thus, there will be YA peers who are responsible for children, often without the other parent's involvement. To promote such peer's job development and maintain their employment, it is important to accommodate their needs, often through flexibility with hours and scheduling.

\section{Greater tolerance of and sensitivity toward diversity}

Today's young adults are less conflicted than older adults about the behavioral norms and values of the various culture groups, and are more comfortable with representations of 
diversity, such as interracial relationships and gay marriage. The higher level of overall tolerance of young adult peer providers can be highly beneficial to provider organizations trying to develop a diverse workplace in regards to race, ethnicity, sexual identity/orientation, and age.

For a general background on this chapter, please see the 2015 Institute of Medicine report: Investing in the Health and Well-Being of Young Adults.

\section{Recognizing and Addressing Workplace Stigma}

Stigmatizing views held by employers and fellow employees make it difficult for young adults with SMHC to enter and succeed in the competitive workforce. Four assumptions strongly underlay workplace stigma: (1) people with SMHC are dangerous to themselves and others; (2) YA with SMHC lack the experience, desire, and social competence to meet the demands of work, (3) working is not healthy for people with SMHC, and (4) providing employment to people with SMCH is primarily an act of charity.xxxiii These views are based on stereotypes: misconceived negative generalizations to individuals in a group bound by shared characteristics or experiences. This kind of stigma is present in many workplaces, including provider organizations, which tend to see clients at their worst, during a crisis or otherwise highly symptomatic. .xxiv $^{-}$

As part of their acknowledged role, peer providers are known by other staff to have a history of mental health problems; right off the bat, this creates a basis for stigmatizing perspectives and attitudes.

In the Table below we address myths associated with these stereotypes, and provide the facts describing the reality of these issues.

\begin{tabular}{|c|c|}
\hline Myths & Facts \\
\hline $\begin{array}{l}\text { People with mental } \\
\text { illness cannot get better/ } \\
\text { recover. }\end{array}$ & $\begin{array}{l}\text { Many people have recovered from mental illness toward } \\
\text { symptom remission and/or social role fulfillment }{ }^{\mathrm{xxx}} \text { Of note, } \\
\text { mental health providers may have misconceptions because } \\
\text { they tend be aware of people with SMHC at their sickest, } \\
\text { and less so when farther along in recovery. }\end{array}$ \\
\hline $\begin{array}{l}\text { People with mental illness } \\
\text { are dangerous. }\end{array}$ & $\begin{array}{l}\text { People with mental illness are on average no more danger- } \\
\text { ous than others. }\end{array}$ \\
\hline
\end{tabular}




\begin{tabular}{|l|l|}
\hline $\begin{array}{l}\text { People with mental illness } \\
\text { are incapable of holding } \\
\text { responsible jobs. }\end{array}$ & $\begin{array}{l}\text { Many successful people who work in responsible positions } \\
\text { have a mental illness, but most have not openly disclosed. } \\
\text { xxxvii Many have been successful because they were able to attain } \\
\text { job flexibility, allowing them to manage their symptoms. }\end{array}$ \\
\hline $\begin{array}{l}\text { Young adults with mental } \\
\text { illness do not have the work } \\
\text { experience or education/ } \\
\text { training to hold a job. }\end{array}$ & $\begin{array}{l}\text { It's true that such young adults have often been forced to veer } \\
\text { off the typical vocational path. But YA peers are able to attain } \\
\text { job success aside from their educational background when } \\
\text { the workplace supports their resilience and persistence. }\end{array}$ \\
\hline $\begin{array}{l}\text { Pexviii } \\
\text { positions are unable to work } \\
\text { full-time because of social } \\
\text { security disability insurance } \\
\text { (SS/D/I) or other limitations. }\end{array}$ & $\begin{array}{l}\text { People on SS/D/I can work full-time but need a job that } \\
\text { supports their basic costs of living and health insurance. } \\
\text { Social security also provides work incentives towards full- } \\
\text { time employment (see chapter 9), and human resource } \\
\text { departments should understand these provisions and } \\
\text { work with peers to maximize opportunities for full-time } \\
\text { employment. }\end{array}$ \\
\hline $\begin{array}{l}\text { Young adults diagnosed with } \\
\text { SMHC are often in crisis. }\end{array}$ & $\begin{array}{l}\text { There is no evidence for such a belief. This bias in the } \\
\text { workplace is often driven by the ways in which TAY have } \\
\text { learned to speak- in the language of needs and symptoms, } \\
\text { which may sound like a "crisis" to supervisors and other } \\
\text { staff. These TAY employees should learn to discuss their } \\
\text { psychological needs in a more strengths based way, being able } \\
\text { to assert what they need to for job success. }\end{array}$ \\
\hline
\end{tabular}

Public stigma in the workplace can induce 'self-stigma', the internalization of stigmatizing attitudes. Young adults with SMHC are particularly prone to self-stigma because of a focus on self-identification with other people in their age group. Self-stigma for young people with SMHC can manifest as feelings of shame, low self-regard, and social unacceptability. For example, peers capable of doing the job may keep to themselves, with only task-related contact with colleagues; this type of social isolation can be seen as a negative by other employees, or they can reach out to the YA peer and establish a social connection (see chapter 8).

Peers, like all employees, depend on good and trusting relationships with other staff for job success. Stigma in the workplace not only invokes negative stereotypes, but also places pressure on employees with SMHC to carefully calibrate how they present themselves to other staff (i.e., what and how much to share about one's SMHC). Stigmatizing attitudes create a climate in which YA peers who are having job-related problems will be reluctant to seek assistance from other employees for fear of being seen as "needy."xxix Thus, stigma often impedes the development of meaningful relationships between peer providers and their supervisors and coworkers. ${ }^{\mathrm{xl}}$ Without an environment of acceptance and inclusion, peer employees may experience increased stress and exacerbation of mental health symptoms, be less likely to disclose their condition toward requesting an accommodation, and be more likely to quit their job. ${ }^{\text {li }}$ 
Stigma is based on ignorance and misinformation, and drives employees' attitudes (prejudice) and actions (discrimination) toward creating social distance from and making negative personnel decisions about people. When there is stigma with regard to mental illnesses, providers will be especially wary about instituting a YA peer provider program in their agency because they expect to face strong resistance from other staff and are not interested in taking the time to address organizational cultural issues. When there is stigma, provider staff will engage in discriminatory behavior through excessively attributing mistakes to the disability, micro-management, gossip, increasing social distance, and limiting opportunities for advancement, and they will be less likely to provide reasonable accommodations (chapter 7).

Thus, providers employing YA peers will need to take a systematic approach toward minimizing stigma. An understanding of the myths and misconceptions of SMCH will provide employees with a basis to self-manage their prejudicial thinking before it turns into discriminatory actions.

However, that is only a start. A stigma-free workplace depends on committed leadership, education and training, exposure to peer providers, and accountability. Throughout this toolkit we describe organizational policies and practices that collectively are meant to support the YA peer, and also to address stigma and discrimination toward peers. Thus, we have devoted the next two sections diversity and anti-discrimination laws. And in Chapter 5 we discuss how to prepare staff to work with peers, and in chapter 9 we address the organizational infrastructure necessary to provide a supportive workplace for peers.

\section{Embracing Legal Obligations To Not Discriminate Against Employees With Disabilities}

Federal and state laws generally prohibit organizations from discriminating against qualified and capable employees with disabilities. (For more specifics on the Americans with Disabilities Act (ADA) and other legal prohibitions, see Appendix B.) These laws prohibit discrimination in all aspects of employment, including job application procedures, hiring, advancement, discipline, firing, compensation, training, and other terms and privileges of employment. Employers breach these laws by maintaining a workplace that is hostile or intimidating for people with mental health conditions (see EEOC commentary).

Although alcoholism and drug
addiction both are disabilities under
the ADA, they are treated differently.
An alcoholic is viewed as having
a disability, as discussed at http://
askjan.org/media/alcohol.html.
Someone who is addicted to drugs
has a disability only if s/he is not
currently using illegal drugs, http://
askjan.org/media/drugadd.html.

Although alcoholism and drug addiction both are disabilities under An alcoholic is viewed as having a disability, as discussed at http:/ Someone who is addicted to drug has a disability only if $s /$ he is not askjan.org/media/drugadd.html.

Disability discrimination laws protect people with mental health disorders, even if symptoms are controlled by medications or by other means (see https://askjan.org/media/psyc.htm). People are also protected under this law when they are "regarded as" having a mental health disorder, even though they might not actually have one. Thus, peer providers are likely to be protected by these laws because they are known to other staff as having had a mental health condition that has impaired their functioning.

Job success for people with mental health conditions is strongly enhanced when both employers and employees understand their obligations to not discriminate against people with 
disabilities. ${ }^{\text {xlii }}$ Awareness of the specifics of these laws itself will inhibit aspects of discriminatory behavior toward people with SMHC ${ }^{\text {xliii }}$ and will help to decrease lawsuits and costly litigation. . $^{\text {xiv }}$ However, organizational employees often know very little about their ADA rights and obligations. ${ }^{x l v}$ Employers should have a complaint or grievance process for reporting discriminatory conduct and investigate these reports to address problems.

Young adult peer employers must clearly and continuously endorse the principles and obligations of the ADA through orientations, trainings, and leadership messaging (see chapters 8 and 9). ADA materials (e.g., booklets) and guidance are readily available online and through federally funded initiatives.

- JAN (Job Accommodation Network) offers a variety of learning materials on the ADA, including web-based trainings, and no-cost confidential advice to both employers and employees on relevant issues.

- There are 10 ADA centers that cover the United States, each responsible for technical assistance and research in their region.

- The United States Equal Opportunity Commission also has good background information on the AD A.

\section{Diversity of Person, Perspective and Experience}

A workplace is diverse when employees at various organizational levels are representative of a larger group of people, such as the local geographic community or its customers/clients. Hiring and other personnel decisions are based on individualized fact- based assessments, and not on stereotypes, resulting in a more dynamic and capable workforce. For a healthcare provider, a diverse workforce improves its overall knowledge of the norms and values of its diverse clientele. It also improves engagement because clients are more likely to trust someone who shares or understands their culture.

People are diverse in multiple ways, such as by age, culture, ethnicity, gender, language, disability, race, religion, and sexual orientation. There have been sufficient levels of workplace discrimination with regard to these demographical characteristics in the U.S. that federal and state laws have been passed to prohibit such discrimination (e.g., ADA). There are many avenues through which employers can discriminate, such as through performance reviews, promotions, salaries, and opportunities to learn or grow on-the-job.

A workplace that embraces diversity has an environment that values, utilizes, and supports the contributions of people with different backgrounds, perspectives, and styles. Such a workplace negates stereotypes and does not tolerate discrimination. It fosters a staff that is interested in and values the perspectives of employees from typically underrepresented groups. The presence of members of these groups working closely with others who do not share a particular perspective or culture will challenge the stereotype that certain categories of people are incapable of assigned tasks.

It will take a strong and sustained effort to promote diversity in an organization. People discriminate because of biases, prejudices, and personal affiliations that limit their experience 
and outlook. And oftentimes people are unaware of their prejudices, which become so ingrained in one's thinking that they feel natural and logical. In addition, group affiliations among staff (e.g., long-time employees, professional groups) can flame workplace prejudice, which is more burdensome on employees hired more recently (e.g., younger people) and in emerging professions (e.g., peers).

There is no quintessential approach for establishing a diverse workplace. There is no evidence that diversity trainings alone impact workplace diversity. But used in collaboration with other initiatives, the best diversity trainings contain positive intergroup interactions towards enhancing the skills, knowledge, and motivation of employees to interact with others who are different from themselves, and who they may not normally talk to on a regular basis. The basic idea is to use multiple approaches, depending on the context of the situation.

\section{Organizational Commitment to Diversity Requires:}

- A conviction among senior leaders that workforce diversity positively affects business performance;

- A strong leadership commitment to diversity planning and implementation (senior leaders must be able to distinguish between diversity management and HR management programs designed to comply with EEO/AA guidelines and requirements.);

- A range of diversity initiatives that encompass more than standard minority recruiting, promotion, and retention programs;

- Formal mechanisms for evaluating and holding company personnel accountable for diversity outcomes;

- Discrimination laws will be observed and adhered to,

o Take immediate action when employees in a work group show disrespect for others.

o Encourage employees to challenge practices that present barriers to different groups,

o Ensure assignments and opportunities are accessible to everyone, and delegate fairly,

o Include employees/co-workers in decision-making processes around diversity,

o Evaluate staff based on their diversity practices and incentivize.

For more information, see Marquis et al., 2008. ${ }^{x v i}$

The Blatt Institute at Syracuse University provides additional useful information on developing workplace diversity policies for people with disabilities, including strategies for communicating and publicizing policies.

In this toolkit, we provide supportive information and tools for promoting diversity. In other parts of this chapter we discuss the importance of employees' understanding and valuing young adult life and culture, addressing mental health stigma, and strategically utilizing employment disability discrimination laws. In other chapters of this toolkit we provide more specific strategies for addressing these issues, in training/supporting supervisors, preparing non- peer staff, and developing agency infrastructure and framework. 


\section{A Positive [Young Adult] Development Approach to Treatmint and Services}

For young adults "positive development" (PD) is a series of goal-directed ongoing activities that generate internal strengths such as initiative, self-reflection, self-efficacy, communication, and problem solving. It is often realized through parental/guardian role modeling, part-time work opportunities, respectful relationships, and safety from violence and other dangers.

PD is an approach now used to empower youth and young adults with disabilities to take an active role in decisions about their lives by improving their executive functioning skills. Executive functioning is the capacity to manage ones cognitive and emotional processes and to strategically develop plans toward accomplishing tasks and projects. With PD, providers offer young adult clients opportunities to set meaningful life goals, while offering them support and direction to develop plans to attain them. Historically, and in a more rigid clinical model, mental illness has been seen as a chronic condition, and young adult clients have been advised to lower their life expectations. Clinicians traditionally have been paternalistic, which is particularly dispiriting to young adult clients who are anxious to develop independent living skills. In similar ways to the peer provider role, the PD approach has clashed with typical clinical practice, which does not focus so much on personal empowerment. Thus, a provider operating primarily from a clinical philosophy may have a very difficult time supporting YA peers.

The Pathways Positive Development model posits that the established intervention is important, but less so than provider factors (See Community-based Approaches for Supporting Positive Development in Youth and Young Adults with Serious Mental Health Conditions).

\section{Critical Provider Skills can be Categorized into Three Domains:}

1. Providers are skilled and able to develop trusting relationships with young adults. The provider brings hopeful and positive energy to the relationship. Critical to building trust is the provider's good listening skills toward encouraging the young adult to share her/his story, opinions, and goals. With relational transparency, the provider does not attempt to coerce or manipulate the young adult.

2. The provider must be very skilled in drawing out young adult's treatment priorities/ perspectives and life goals, and helping them to identify feelings of ambivalence and conflict.

3. PD based practitioners motivate clients to learn and practice their metadevelopmental skills, enhancing the client's competence, and self-efficacy. The provider here does not simply engage the young adult in a planning process, but more importantly teaches him/her assessment and decision making skills they can use to improve their standing in life.

For more information, see also: 
Theory and Research Underpinnings Supporting the Transition to Independence Process (TIP) Model

Healthy Transitions: The Provider Toolkit for Emerging Adults with Serious Mental Health Conditions [Georgia]

The RENEW Model: Supporting Transition-Age Youth with Emotional and Behavioral Challenges

\section{Self-Determination And Dignity of Risk}

Self-determination is the ability to make decisions and choices based on one's own values and preferences. xvii "Dignity of risk" is respect for each individual's autonomy and self-determination to make poor choices even though at times the risks may appear to outweigh the benefits. For young adults, making personal choices generally facilitates long-term growth through identifying strengths and weaknesses, experiencing life's challenges, and learning from one's mistakes. ${ }^{\text {xlvii }}$ With regard to adult mental health services, provider organizations and individual clinicians are often risk averse, concerned that when left to their own devices, young adult clients will make poor decisions that risk their health. Provider staff are thus wary about young adults' making their own treatment decisions, even when they've shared the benefits and side effects of treatment. A culture and staff that endorses this approach will conflict with the goals and aims of the YA peer, which in part is to support client self-determination. In this environment, YA peers may often disagree with the staff decisions around client participation in treatment, making working conditions difficult for them.

On the other hand, YA peers will thrive in a culture that aims to realize the concepts of selfdetermination and dignity of risk. In such cultures, YA clients are presumed to be competent and are entitled to make their own decisions. Here, practitioners encourage clients to take "risks" (e.g., attending social gatherings, going back to school) and try out new choices (e.g., learning to drive). When organizations openly value these concepts, the YA peer will be in a better position to advocate for client preferences with their non-peer professional colleagues, including the right for clients to make treatment decisions that appear to be risky but have been thought out (e.g., no longer taking a medication with undesirable side effects). Providers who value selfdetermination usually struggle with giving full autonomy to clients, and facing the dilemma of agreeing to a treatment choice that goes against their better judgment. Most peers will appreciate the providers' concerns, and will ask the provider to balance short-term risk with long-term gains of developmental learning and identity formation.

Many providers report their agreement with self-determination notions but struggle to put them into practice. In these cases, there are opportunities for peers to thrive as advisors on engaging YA clients in treatment decision making. YA peers can thrive when providers are welcoming to their advice on policy and practice development.

As stated earlier, the community-based Wraparound approach for families includes the active 
engagement of parent(s) and youth partners in treatment delivery. However, older adolescents and young adults typically show less interest in Wraparound because of their desire for more autonomy (i.e., in relation to parents). ${ }^{\text {xix }}$ Various Wraparound programs have begun to address this issue with specific strategies to generate young adult self-determination through youthdriven family informed approaches, such as integrating the "futures planning" process into the Transition to Independence Process (TIP) model (chapter 1).

To address YA self-determination in Wraparound, Massachusetts has utilized a SAMHSA grant to introduce the YA peer role into the transition-age youth Wraparound process (and are now sustaining the process through Medicaid reimbursement as Therapeutic Peer Mentors). A challenge for YA peers has been bridging the gulf between the perspective of parents on the limits of young adult client autonomy and the client's interest in making one's own choices. A culture of self-determination will respect the parents' position, value other perspectives, and recognize the primacy of the young adult client's right to autonomy. Providers who value self-determination train and support their staff, including YA peers, to engage in a dialogue with parents and youth about the need for shifts in youth autonomy and control to occur in order for recovery to take place.

\section{Employee Access to Wellness Tools, Services and Supports}

Human services jobs are generally stress-inducing because of the significant performance pressure, often with limited resources and low pay. Workplace stress directly relates to poor health and disability, and translates to absenteeism, poor performance, and job loss.

Young adult peers succeed through building positive psychological capital. Positive psychological capital is characterized by a person's "self-efficacy, optimism, perseverance, hopefulness and resilience."li An agency boosts psychological capital by embracing a culture of health and wellness, with a range of options to prevent and address work-related stress, as well as investing in the development and advancement of their employees.

Organizational leadership is responsible for maintaining this culture and can meet this responsibility by providing access to good healthcare and insurance coverage. For additional information on the employer's responsibilities for providing behavioral healthcare coverage under the federal parity act legislation, see Employer Guide for Compliance with the Mental Health Parity and Addiction Equity Act.

Some health supports are accessible to most provider employees by law. This includes:

- The Reasonable Accommodation (RA) requirement of federal and state anti-discrimination laws (chapter 7);

- The Family and Medical Leave Act requires covered employers to grant employees 12 weeks of leave per year to address serious health conditions. This is a federal law but several states have passed laws to enhance its coverage.

For YA peers, there are many additional employee supports and programs that providers include 
to boost the psychological capital (i.e., on-the-job resilience), and the overall wellness of their staff. Here are a few that we find particularly beneficial:

\section{Key Initiatives to Promote Workplace Wellness}

- Employee Assistance Programs (EAP) typically provide wellness supports for employees and assist them in accessing health services, and are more likely to exist in larger companies. As a confidential counseling and referral service for employees experiencing mental health struggles, ${ }^{\text {lii }}$ EAPs can help employees retain their jobs by connecting them to evidence-based services and supports shortly after symptoms begin. ${ }^{\text {lii,liv }}$ The American Psychiatric Foundation's Partnership for Workplace Mental Health has published a helpful guide for how EAP staff can effectively address mental health problems: Employer Practices for Addressing Stress \& Building Resilience.

- Emerging as a lower-cost alternative to EAPs, Employer Resource Networks (ERNs) are multi-employer collaborations designed to link and leverage vocational support resources.

lv Some ERNs are developed by groups of businesses that pay a fee to participate, while others are based on government subsidies, such as Massachusetts' five "Regional Employment Collaboratives." viv These collaboratives help organizations to hire and retain people with disabilities.

- Short- term Disability Insurance (STDI) pays a portion of the salary (usually between $50-75 \%$ ) to an employee who becomes "disabled" (per policy requirements) and takes leave as a result. lvii This allows the employee to fully focus on his/her recovery toward retaining the position for the long-term. The evidence to date suggests that when an employer provides STDI benefits to employees with serious mental health conditions and helps them to access evidence-based services and supports, workdays missed for this segment of the workforce decreases and productivity increases. ${ }^{\text {lvii }}$

- Outreach and Cognitive Behavioral Therapy (CBT) based return-to-work programs, including practical strategies to manage stress. The CBT approach to workplace integration focuses on understanding the causes of workplace stress and developing practical strategies to better manage it. ${ }^{\text {lix }}$ Studies show that cognitive- behavioral programs consistently produced larger effects than other types of interventions. ${ }^{\text {lx }}$ Web-based CBT programs have proven to be successful, particularly when the occupational physician is able to make automated suggestions for treatment referrals based on user-provided information about progress. ${ }^{\text {lxi }}$

- Resilience building programs help YA peers to improve their self-care and stress management. Successful programs assist participants in reframing negative thinking, developing lifestyle habits that enable coping, and for some, developing wellness/crisis action plans for the workplace. These programs often focus on exercise, relaxation,

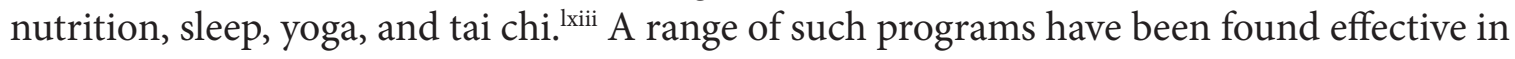
reducing psychological symptoms (e.g., depression), absences, and job loss.

- Coaching and mentoring programs that focus on job development and career oriented goal setting. Coaches offer a range of cognitive and behavioral techniques through regular meetings to help an employee develop his/her short and long term goals. This approach has been associated with improved worker well-being, fewer depressive symptoms, and increased job satisfaction. (See Creating a coaching plan toolkit and Coaching for on the job development). 
- Human Resource departments can support employees with job concerns through webbased "hubs" that provide information on workplace supports, such as reasonable accommodations, accessing health services, and SSDI eligibility.

- Worksite physical activity programs can reduce stress in employees when they engage in

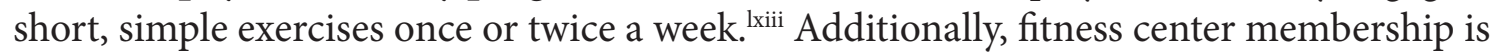
associated with increased health responsibility and health promoting behaviors.

\section{- Opportunities for YA peers to decompress on-the-job}

o Maintain a physical space for YA peers to take a break, consult with one another, and/ or to meet with clients privately when necessary;

o Provide regularly scheduled support and discussion meetings for YA peers across programs and agencies; and

o Have YA peers attend trainings where groups YA peers gather.

- Involve peers and workers in assessing workplace stress through a variety of venues. Surveys, town halls, discussions, or focus groups are great ways to understand employee stress and burnout, and also to generate ideas of how to improve the workplace culture to combat these negative work experiences. ${ }^{\text {xiv }}$ YA peers should be active participants on workplace wellness task forces and committees.

\section{Additional Resources:}

Very good guidance on workplace wellness best practices can be found in a 2014 report of the Australian National Mental Health Commission and the Mentally Healthy Workplace Alliance: Developing a mentally healthy workplace: A review of the literature.

Additionally, the federal Equal Employment Opportunity Commission EEOC has provided important information on the impact of the ADA on wellness programs, particularly in collecting disability related information, incentivizing wellness goals, and the use of genetic information in this report: Guidance to employers on Employer's wellness programs in relation to the ADA). A helpful summary of organization wellness requirements that address these ADA concerns can be found through the Kaiser Family Foundation: Workplace Wellness Programs Characteristics and Requirements. ${ }^{\text {lxv }}$ 


\section{5 | Recruiting, Hiring \& TRAININg Successful Young Adult Peers}




\section{5|RECRUITING, HIRING \& TRAINING SUCCESSFUL YOUNG ADULT PEERS}

\section{Recruiting And Hiring}

1. Define "Peer" in your provider context

2. Describe key functions of the YA peer position

3. Establish job qualifications

4. Written job description for the peer provider role

5. Young adult peer job classification and pay

6. Find and attract successful young adult peer provider candidates

7. Interviewing young adult peer candidates

8. Other things to consider in the process of identifying, recruiting, and interviewing young adult peer provider candidates

\section{Define "Peer" in your provider context}

Consider the youth and young adult clients that you serve or desire to serve. Ask yourself, who are your YA clients' peers? What kinds of characteristics, expertise and/or life experiences should they have and not have? What peer characteristics, expertise and/or life experiences would fit well with your agency/context? What characteristics, expertise and/or life experiences are missing or needed among your peer and non- peer staff?

Here are some examples of characteristics \& experiences peers and clients might have in common:

- Near in age

- Mental health concerns, diagnoses, and/or substance use challenges

- Vocational experiences and/or goals, such as having been assigned an Individual Education Plan (IEP), delayed high school completion, struggles with post-secondary education, and difficulty securing and/or retaining employment

- Interests, hobbies, and career paths

- Treatment experiences, including residential care, psychotropic prescription medication (including injectables), psychiatric hospitalization, and/or outpatient care

- Adverse childhood experiences, including instability at home, isolation, bullying, trauma exposure/abuse, and/or grief and loss

- System involvement - child welfare, juvenile/criminal justice, special education

- Demographic characteristics: race/ethnicity, gender, sexuality, \& community affiliation

- Treatment

\section{Describe key functions of the YA peer position}

There are, of course, functions essential to any YA peer position (chapter 1). In addition, 
the peer job may have a focus on specific client populations that will impact the necessary functions, and thus job qualifications and recruiting. These areas of focus have included (but are not limited to) forensic involvement, dual disorders, employment, housing, care navigation, and community re-entry.

\section{Establish job qualifications}

The primary job qualifications for the YA peer job should relate to applicants':

- Lived experience;

- Capacity to engage in peer support with clients;

- Capacity to advocate with and for clients;

- Professional and ethical conduct.

Job qualifications may also relate to the completion of specific trainings and the attainment of peer specialist certification, as discussed below.

Of note, many providers have found that the application of common job criteria for care positions can effectively screen some of the most promising YA peer job candidates. Job direct criteria that most frequently screen out young adults with SMHC are educational attainment and criminal history. Many of these candidates are optimal for a given position because they have overcome high barriers toward their recovery and can best relate to clients facing similar struggles (e.g., forensic peer specialist). Thus, it makes sense for program management and HR to:

- Relax direct service requirements that tend to screen out TAY applicants (e.g., criminal history, education). This includes the minimization of exclusionary criteria that could be better addressed on a case-by-case basis.

- Establish new criteria in place of the limiting requirements by determining:

o The degree to which criteria impacts job performance;

o The criterion's relevance in relation to other peer job qualifications, such as lived experience;

o The extent to which the applicant has overcome barriers.

Educational requirements typically applied to direct care workers often exclude capable YA peer applicants from job consideration. It is not uncommon for agencies to require that employees have at least a high school diploma or college degree before they will even be considered. Many YA peer applicants have not yet attained these degrees but do have essential experience in overcoming educational challenges that is reflective of current clients' needs:

- Their lived experience of facing serious educational difficulties and achieving a state of wellness in spite of those difficulties;

- Their continuing efforts to advance their education while managing their mental health condition;

- The hard lessons they have drawn from these experiences.

Thus, providers should assess the degree to which their educational requirements are excluding good candidates, keeping in mind the relevance of peer training classes and certification. 
Providers typically conduct a criminal background check for direct care job applicants in order to evaluate, or screen out, applicants with convictions for various offenses. Such practices create employment barriers that disproportionately affect members of disadvantaged groups, including the many TAY who have had contact with the criminal justice system for arrests and/or convictions for minor offenses. However, peer applicants with criminal histories are often the most empathetic and experienced when talking to another peer facing serious struggles and impediments, especially those within forensic or diversionary settings.

The Equal Employment Opportunity Commission (EEOC) now discourages the use of employment exclusionary criteria based on criminal justice involvement in favor of a more individualized review of each candidate (see the 2012 U.S. Equal Employment Opportunity Commission (EEOC) guidance). Based on the EEOC guidance, several best practices have emerged to guide employers in offering people with a criminal background a fair chance for employment (see Best Practices and Model Policies: Creating a Fair Chance Policy, of the National Employment Law Project).

\section{Best practices on criminal history when hiring TAY job applicants:}

- Criminal history as a hiring factor

o Avoid exclusions related to criminal history, except when legally mandated;

o An applicant's criminal record should be factored into hiring decisions only when directly related to job duties and responsibilities, and then in light of the gravity of the crime and the length of time since the offense;

$\mathrm{O}$ If there is a concern about a criminal conviction, provide the applicant with the right, and sufficient time, to submit evidence of mitigation or rehabilitation. (Background check reports can be inaccurate.)

- $\quad$ Job applications and notices

o The inclusion of a check off "box" or any other query about all or some criminal convictions often discourages people with any criminal history from applying. Thus, do not ask about criminal history on job applications (there will be chances to ask relevant questions during the interview process). (Of note, as of April 2017, over 100 counties and cities as well as 25 states have adopted "Ban the Box" policies in their Fair Employment laws and regulations.)

o Do not include a provision to permit "voluntary disclosure" of background check information from the applicant.

o Generally avoid phrases such as "must pass a background check," or "clean background only" as this language may be interpreted as a categorical exclusion.

o If a background check is necessary or if there is a specific legal barrier, state that on the application.

- Expungement

Some very qualified candidates may have a record of relatively minor criminal convictions that by state laws/ regulations or agency policies exclude them from employment. Often YA job applicants have limited knowledge of what is in their record and may be upset or surprised to learn the types and number of charges.

We advise companies to help qualified candidates to attempt to clear (expunge) their record through an appeal process or waiver available in many states (see 50 state guide to judicial relief). Companies can directly support the job applicant in this expungement process and/or refer the candidate to other available assistance. 


\section{Written Job Description for the Peer Provider role}

Develop the YA peer provider job description (and complete the three previous steps)

BEFORE hiring takes place - even if you have found the perfect young adult to be your new "peer provider." There will be opportunities to alter the description as more is learned about how to effectively implement the role; however, a job description is a necessary starting point in providing staff and potential peer applicants with basic planning information for:

- $\quad$ Potential peer applicants - to determine if it's a job they really want and then what supports they will need in place;

- Management - to plan for implementation and support the role;

- Existing staff- to better understand their roles and responsibilities in relation to those of the peer;

- $\quad$ Peers when hired - for job clarity and direction.

Have your HR department, administration, and staff who will be working with YA peers involved in shaping the role and determining key desirable characteristics. By being part of this process, non- peer staff will learn about the role and feel a sense of commitment having been a part of creating it - thus decreasing potential conflict about the role down the road. For a sample job description, see Appendix C.

\section{Young Adult Peer Job Classification and Pay}

Job classification systems aim to define and evaluate responsibilities, functions, and authority levels of each job. These systems place job types in a class based on functions and requirements, and each class often has a series of grades, which include compensation ranges, other benefits, and promotion opportunities.

We find that most provider agencies, when first considering hiring peers, do not have job classification systems that include the peer job title or something analogous in terms of responsibilities and skills. This may delay hiring or result in peers being placed into job classes that under-represent the level of responsibility they take on, with negative impacts on salary and benefits. Thus, program leadership should bring in HR well before hiring to review the YA peer description and to link the job to the appropriate job classification.

We do believe that because the peer job has its own unique features, skill sets, and qualifications, it makes sense to have a single class focused on this growing professional role. The challenge is that job classification systems have often been negotiated among multiple parties, including HR, clinical leadership, and sometimes unions. Under these circumstances, job titles and descriptions can be difficult to alter or re-create. But when program leadership, in advance of hiring peers, discuss with other company departments and union leadership about the need for a "peer" job classification, the potential for creating that classification is greatly enhanced. In fact, union contracts often allow for the creation of new job types, but time for education and negotiation is needed. As with any other profession, compensation should reflect the skills required for the particular peer role, as a matter of fairness and in order to attract high quality candidates. 


\section{Find and Attract Successful Young Adult Peer Provider Candidates}

For effective YA peer recruitment, you must establish connections with networks that YA peers are associated with, including: youth and young adult peer and advocacy groups, vocational training programs for older youth/young adults with disabilities, treatment programs, and local post-secondary education programs, including certificate programs, associates, and bachelors programs. It is especially important to distribute job notices on social media and blogs that potential candidates follow. The job notice document must have language that is youth/young adult appropriate and be in a format that can be forwarded via email.

\section{Interviewing Young Adult Peer Candidates}

Basic interview guidelines to follow are: ${ }^{\text {xvi }}$

- Review the job description with the YA peer candidate;

- Advise the YA peer candidate that he or she must be able to talk about their recovery;

- Make clear that it is essential that the candidate be able to serve as a role model of recovery;

- Ask about prior work history.

According to the EEOC, employers are prohibited from using qualification standards, employment tests, and other selection criteria that screen out or tend to screen out individuals with disabilities, unless the criteria relates to a necessary qualification or job function. Since the peer role generally requires that the job-holder have the lived experience of a mental health condition and/or have used mental health services, inquiries about these topics in relation to job functions is permissible (See 2007 bulletin of the EEOC). CASRA, in their guide Meaningful Roles for Peer Providers in Integrated Healthcare provides examples of valid interview questions (page 115):

1. "Please tell us about what recovery means to you and how your own experience informs your knowledge and beliefs?"

2. "One of the essential job functions of this position is being able to share the story of your recovery from behavioral health challenges for the benefit of consumers/family members receiving services. Can you give us an example of using your story to benefit a client or family member who is struggling with an issue?"

3. "What do you feel is the most important aspect of peer-provided services?"

Specific questions not tied to essential job functions-about diagnoses, medication use, and experience of treatments-are generally not permissible. However, questions about how a YA peer would maintain healthy relational boundaries on the job are valid to explore. See the EEOC's helpful resource: Pre-Offer, Disability-Related Questions: Dos and Don'ts (https:// www.eeoc.gov/eeoc/publications/adahandbook.cfm\#dodonts) for additional guidance.

An applicant may ask for a reasonable accommodation to help them apply for the job (such as having a job advisor present at the interview with them). An excellent resource for providng accommodations during the job application process is at the Job Accommodation Network- Employers' Practical Guide to Reasonable Accommodation Under the Americans 
with Disabilities Act, page 7. And as noted above, ask criminal record related questions only when it is directly tied to the qualifications and requirements necessary for the job. The most effective policy is to delay all such inquiries until after a conditional offer of employment is made.

\section{Other Things to Consider in the Process of Identifying, Recruiting, \& Interviewing Young Adult Peer Provider Candidates}

For young adults specifically, it is very important to assess not only their commitment to the role, but their availability to do the job, as many young people are juggling work, school, and other commitments. It is also important to assess the extent to which the young adult is truly

a peer of your provider's clientele. In regards to considering what kind of training and support will be necessary for YA peer candidates, consider how professional the candidate acts as well as the extent to which they can work autonomously. The questions listed below will help your organization to identify ideal YA peer provider candidates.

\section{Commitment to YA Peer Role \& Availability}

- Why is the candidate interested in being a peer provider? How motivated is the candidate to secure this position and share their lived experience to motivate their peers?

- How long is the candidate likely to serve in this role? (At least a year commitment is desirable given that changing jobs/moving is common during this period of development).

- What kinds of hours can the candidate dedicate to being a YA peer? (Especially, what is the candidate's availability during the day, evenings, and weekends? Can the candidate make the weekly team meeting?)

- Is the candidate employed elsewhere or going to school? Will the candidate continue with these commitments? Are there any other current responsibilities that the candidate has that will likely be inspirational/motivational to current clients (such as caretaking, volunteering)?

- What ideas do they have for activities to engage youth and young adult clients? How Creative are they? How comfortable are they doing outreach? Are they willing to meet with clients both in the community and on-site at the agency?

\section{Peer Characteristics \& Attributes}

- What shared "peer" characteristics, other than having mental health challenges and experience with treatment, does the candidate share with the agency's younger clientele?

- How well will YA clients be able to relate to the candidate? Conversely, how well do you think the candidate will be able to relate to the agency's younger clientele?

- What is the candidate's story? How will the candidate share their story with younger clientele? Have they shared their story before?

- Is the candidate able to convey hope? What are their personal life goals? Do they see this position as a stepping-stone to their long-term career goals?

\section{Professionalism \& Capacity for Autonomy}

- How confident does the candidate appear? Will the candidate remain resilient on the job? How does the candidate handle stressful situations?

- How much supervision, training, and/or support will the candidate need in the YA peer role?

- Is the candidate an effective communicator, and of note, what kind of support would help him/her get there? Different types of communication include email, text, and face-to-face. 
Does the candidate communicate well with peers \& supervisors/senior staff? Do they have experience in documentation/clinical note writing? (Have the candidate engage in a writing activity during the interview process.)

- What concerns does the candidate have about their competency to perform the duties that are part of the YA peer role?

\section{Training Young Adult Peers}

Basic understanding of your workplace. In relation to older peers, young adult peers are more likely to need assistance learning about workplace norms and expectations. An array of trainings that would be important to the success of many YA peers includes:

- Essential workplace skills;

- Personnel policies and benefits;

- Workplace rights and responsibilities;

- Building resilience through stress management, self-care, and wellness planning. Ixvii

There are many free resources online that supervisors and training staff can build on in order to tailor trainings to meet the specific needs of YA peers in your context.

Reinforce Required Staff Trainings. YA peers should participate in all agency required orientation trainings, which should include an introduction to the following: agency procedures (e.g., benefits), company culture and philosophy, and how to use agency resources. In order to begin a dialogue about agency policies and practices that is transparent from the start, the YA peer's supervisor should revisit these topics after the initial orientation meeting. Group supervision is an excellent forum for inviting human resources and benefits staff on a quarterly basis to provide information, and reinforce discussions about benefits, policies, and work-related information - all of which YA peers can gain competency in so that they can coach their youth and young adult clients to inquire about these topics when they gain employment.

Additional training topics that build YA peer job skills:

- Motivational Interviewing \& Active Listening

- Professionalism

- Healthy Boundaries

- Strategic Sharing

- Suicidality assessment \& safety planning

- Communication and leadership styles

Access to YA Peer-specific Trainings. There are a growing number of training opportunities available for YA peers to develop skills and competencies. Most states offer the Certified Peer Specialist (CPS) training, which educates attendees on the basics of being a peer provider, with a focus on the profession's ethical standards and specific approaches to engaging clients in the recovery process. ${ }^{\text {xviii }}$ States and providers often favor certification because, in some cases, it allows the provider to bill Medicaid for services while ensuring that the YA peer has an understanding or 
their job responsibilities. The CPS training is intensive (typically 80 hours in length) and requires the passing of an exam in order to become "certified" as a peer specialist. ${ }^{\text {lxix }}$ We recommend that young adult peers take this training, or a version of this training, that addresses specific young adult issues. Other valuable trainings for YA peers include Wellness Recovery Action Planning ${ }^{\odot}$ (WRAP) ${ }^{\circledR}$ and Whole Health Action Management (WHAM) trainings. Both will provide the YA peer provider with concrete tools to engage and partner with young adult clients around their health management goals. Peer run trainings for the WRAP and the WHAM are hosted in many states and counties. With government support they tend to be low in cost or free and are between 1 to 3 days in length.

YA peers report that the best trainings teach peers: ${ }^{\mathrm{Ixx}}$

1. That the client is the expert on his/her own care;

2. Strategies for helping people diagnosed with SMHC to take control of their lives;

3. To not depend on any one, specific engagement approach (e.g., "to meet clients where they are").

Organizations should leverage their relationships with other organizations, and state and national entities, in order to establish ongoing trainings, workshops, and conference opportunities for YA peer providers to interact with other YA peer providers. This serves two functions: 1) to develop skills that are beneficial in their work as YA peers and 2) to develop a natural support network of YA peer providers. By connecting with others who are trying to engage a similar population and are facing similar challenges in their role, YA peer providers experience a strong sense of validation through collective mentoring, natural social support connections, and learning directly from their peers.

\section{Teach Principles of Strategic Storytelling}

Storytelling is a form of art that appeals to our emotions. Strategic sharing is an approach to sharing one's life experiences in order to influence and instigate change. While storytelling comes more naturally to some, it is a skill that can be developed over time among YA peer providers, and it is necessary to effectively engage and impact their clients. Strategic storytelling itself is a reflective process where young people: 1) learn how to develop motivating stories; 2) engage in sharing personal experiences with key people at key times; and 3) process these sharing experiences in supervision in order to maintain on-the-job resiliency and hone strategic storytelling skills.

\section{Key Elements for Effective YA Peer Provider Strategic Storytelling}

- YA peers must develop confidence and competency in strategic sharing, as it is a key part of their job. It is the provider's responsibility to ensure that YA peer providers are prepared and supported in this crucial part of their role. Providing regular training workshops on, and supervision around, storytelling is important. Storytelling workshops should ideally include peer and non-peer staff, especially peer provider supervisors.

- Stories must be understood as dynamic, not static. Stories change as we develop and create 
new meaning of life experiences. An especially important notion to embrace is that a story that is emotionally triggering today might not be so tomorrow, and vice versa.

- In order to be effective, storytelling workshops should be augmented with training in active listening and motivational interviewing. It is especially important for YA peer providers to learn the power of non-judgmental, open-ended responses when engaging youth and young adult clients. YA peer providers should learn clear ways to express empathy and genuine concern for their clients.

- Supervisors and YA peer providers should recognize the risks associated with strategic storytelling for young adult peer providers and ensure that they have sufficient on-the-job support. Sharing one's life experiences, even the positive ones, may produce unintended information and feelings in YA peers as well as unintended reactions from clients.

- Those who train and/or supervise YA peers must apply a strengths-based approach in supporting them in reframing their personal life struggles into stories of overcoming hardship that will inspire their clients. This process is akin to narrative therapy, where various aspects of a story are explored and reframed to empower individuals and families.

- As YA peer providers participate in initial trainings, they learn to use their experiences to engage youth and young adult clients.

\section{Two fantastic resources for understanding the basics of strategic storytelling are:}

1. The Pathways Research \& Training Center Strategic Sharing Workbook: Youth Voice in Advocacy. This tool is designed to increase competency of peer provider supervisors in their efforts to support YA peers in developing their peer provider worker identity. Accessible at: http://www.pathwaysrtc.pdx.edu/pdf/pbStrategicSharingGuide.pdf.

2. The Hunter School of Social Work Strategic Sharing, which teaches young adult peers to consider what elements of the story to share to certain audiences as well as the importance of personally claiming the meaning of the story being shared. Clients will make sense of peer provider stories in various ways - and it is very important that the young adult peer provider claim their emotions and perspectives of their personal stories. Accessible at: http://www. hunter.cuny.edu/socwork/nrcfcpp/info services/download/StrategicSharing.pdf.

\section{Review and Discuss Peer Provider Disclosure Policies}

Disclosing personal information is at the heart of the YA peer provider role, but has particular implications for young adults. Social media (e.g., Facebook, Twitter, Instagram), online video sharing websites (e.g., YouTube, VineVideos, Vimeo) and agency websites that integrate YA peer photos, videos, and stories are methods that are likely to engage youth and young adult clients. Your organization must work with young adult peer providers individually to establish the extent to which the young adult peer provider wants their personal identity (e.g., name, photo) shared on organization websites and social media outlets. This is beyond seeking YA peer consent; it is ensuring that a young adult peer understands that what goes on to the internet may never truly be removed.

Over time, YA peers may change their perception of having their name and photo (or video) 
associated with a particular organization or cause. The supervisor must continue to discuss organizational use of young adult peer provider personal stories for marketing or advocacy purposes with both YA peer providers and organizational administration. Best-practices for online information sharing for YA peer providers includes having photos, quotes, and videos on the internet for a fixed amount of time to allow multiple YA peer providers the opportunity to be in the spotlight, but also limit the time that a YA peer provider's name, image, and story are available online. 


\section{6 | EfFective SuPERVISION FOR Young Adult Peer Providers}




\section{6|EFFECTIVE SUPERVISION FOR YOUNG ADULT PEER PROVIDERS}

\section{Sections:}

- Principles of Young Adult Peer-Supervision

- Essential Supervision Topics

- The Importance of the YA Peer-Supervisor Working Alliance

- Bolstering YA Peer On-the-Job Resiliency

- Training, Supervision, and Support for YA Peers

- Moving On

\section{Principles of Young Adult Peer Supervision}

Supervision of young adult (YA) peer providers ("peers") is effective when it is scheduled in advance and occurs regularly: the same time and place each week or bi-weekly. In order for them to be successful at their position, it's important that YA peers have a specified time and place to process and reflect upon their work experiences. Ideally, YA peer providers partner with supervisors to set the supervision agenda. Time should be set aside at the start of supervision to follow up on anything identified as pertinent from the previous supervision meeting.

Supervisors must be aware of the concurrent need for reflective supervision and skill development when supervising young adult peers. Both individual and group supervision can be beneficial for young adult peer providers. Ideally, YA peers meet weekly for individual supervision with their supervisor for 30-60 minutes, and bi-weekly for group supervision for 60 minutes. In general, it is important to support YA peer providers in connecting with other YA peer providers whenever possible. Group supervision can be a particularly powerful experience for mutuality in peer-topeer relationships, as well as a fantastic forum for skill development through mini-workshops hosted by supervisors, guest trainers, and the YA peers.

Beyond supervision competencies, it is critical for the YA supervisors to understand the content of the previous Toolkit chapters, particularly the purpose and responsibilities of YA peers (chapter 2 ), the impact of their introduction on non peer staff (chapter 2), and relevant organizational cultural elements (chapter 4).

You might be curious about how young adult peer provider supervision might differ from adult peer provider supervision. Below we have expanded on key identified adult peer supervision principles $^{\mathrm{lxxi}}$ and describe how they can be interpreted for the supervision of YA peers 


\section{Young Adult Peer Supervision Principles}

\begin{tabular}{|c|c|}
\hline $\begin{array}{c}\text { Pillars of Supervision } \\
\text { Principles }\end{array}$ & Expansion for YA Peers \\
\hline $\begin{array}{l}\text { 1. Peer Specialist } \\
\text { Supervisors are Trained } \\
\text { in Quality Supervisory } \\
\text { Skills. }\end{array}$ & $\begin{array}{l}\text { - Supervisors need additional training in developmental milestones } \\
\text { of the transition to adulthood, positive youth development, and } \\
\text { engagement techniques, in order to effectively supervise and } \\
\text { empower YA peers. } \\
\text { - Giving and receiving feedback to YA peers may look different } \\
\text { based on the YA peer's experience with supervision, direction, } \\
\text { and guidance. Supervisors need to be flexible in their approach to } \\
\text { supervision }\end{array}$ \\
\hline $\begin{array}{l}\text { 2. Peer Specialist } \\
\text { Supervisors Understand } \\
\text { and Support the Role of } \\
\text { the Peer Specialist. }\end{array}$ & $\begin{array}{l}\text { In addition to supporting continuing education and certification, } \\
\text { supervisors must learn how to support the development of } \\
\text { strategic sharing skills for YA peers. } \\
\text { - Supervisors must be readily accessible and available for } \\
\text { processing potentially traumatic or confusing situations that their } \\
\text { YA peer providers could encounter while working. } \\
\text { - Supervisors understand today's young adult experience and } \\
\text { culture, including trends in social media use (chapter 4). }\end{array}$ \\
\hline $\begin{array}{l}\text { 3. Peer Specialist } \\
\text { Supervisors Understand } \\
\text { and Promote Recovery } \\
\text { in their Supervisory } \\
\text { Roles. }\end{array}$ & $\begin{array}{l}\text { - Recovery looks different for transition-age youth (TAY) - both } \\
\text { YA peers and TAY clients. Rather than focusing on "recovery," } \\
\text { supervisors must focus on "discovery" and support YA peers' } \\
\text { efforts to engage YA clients in exploring their contexts, identity, } \\
\text { and symptoms, through sharing their personal stories. } \\
\text { - Supervisors must be trained in Positive Youth Development } \\
\text { (chapter 4). } \\
\text { - Supervisors must also teach YA peers how to monitor stress } \\
\text { levels. } \\
\text { - Supervisors should celebrate their YA peer providers' milestones } \\
\text { at work in young adult-friendly ways. } \\
\text { - Supervisors must teach and model self-care to YA peers. } \\
\text { - Supervisors should focus on the positive while addressing } \\
\text { barriers/challenges. }\end{array}$ \\
\hline
\end{tabular}




\begin{tabular}{|c|c|}
\hline $\begin{array}{l}\text { 4. Peer Specialist } \\
\text { Supervisors Advocate } \\
\text { for the Peer Specialist } \\
\text { and Peer Specialist } \\
\text { Services Across the } \\
\text { Organization and in the } \\
\text { Community. }\end{array}$ & $\begin{array}{l}\text { - Promoting mutual trust, respect, and collaboration between } \\
\text { YA peers and other staff will be challenging, because of a } \\
\text { combination of stigma toward both "peer provided services" and } \\
\text { "youth culture." When staff criticize YA peers, supervisors must } \\
\text { champion efforts to explain that they are at the agency to work } \\
\text { like anyone else, and we need to get to know them better } \\
\text { - In order to demonstrate the value of YA peers, supervisors need } \\
\text { to document the impact that they are having with their individual } \\
\text { clients and on an organizational level (see chapter } 2 \text { for more } \\
\text { detail on these benefits). }\end{array}$ \\
\hline $\begin{array}{l}\text { 5. Peer Specialist } \\
\text { Supervisors Promote } \\
\text { both the Professional } \\
\text { and Personal Growth } \\
\text { of the Peer Specialist } \\
\text { within Established } \\
\text { Human Resource } \\
\text { Standards. }\end{array}$ & $\begin{array}{l}\text { - Identity, relationships, and career exploration are key parallel } \\
\text { processes for YA peers to experience as they guide and support } \\
\text { their YA clients. Supervisors must be aware of this and promote } \\
\text { YA peer exploration. } \\
\text { - Supervisors must form a strong working alliance with YA peers to } \\
\text { support exploration and safety on the job. } \\
\text { - Supervisors must educate Human Resources about the value that } \\
\text { YA peers bring to the organization and the reasons some general } \\
\text { requirements for direct service staff may need to be relaxed. }\end{array}$ \\
\hline
\end{tabular}

From: Pillars of Peer Support - VI: Peer Specialist Supervision (2015)www.pillarsofpeersupport.org.

In addition to the principles discussed above, here are best-practices for young adult peer provider supervision:

- Balance the Negative with the Positive and Celebrate Small Successes. Model strengthsbased approaches for YA peers to use with clients. It is important to recognize the small successes of YA peer providers so they can learn how to recognize small successes in their YA clients. In addition, young adult peers are at high risk for feeling discouraged because they do not perceive their clients as engaging or progressing. By celebrating small wins, such as trying out new skills with young adult clients, young adult peer provider on-the-job discouragement can be averted. For example, when a peer and client establish a weekly meeting time and place and get together a few times in a row, a supervisor should recognize the peer for successfully engaging the client.

- Supervisors/Senior YA Peers Must be Present and Available. Frequent informal check-ins and supervisor/senior peer staff availability for impromptu check-ins are key for on-the-job young adult peer provider success. These check-ins foster security on and off the job site and can happen via in-person interactions, phone calls, or texting exchanges. An excellent practice that provides for YA peer leadership development is to have more senior young adult peers mentor junior young adult peers, while also providing effective on-the-job support. The check-in should not feel like "monitoring", but instead like a consultation.

- Outreach to Young Adult Peer Providers May Be Necessary at Times. Check in with young adult peer providers whose behavior and communication has changed at work, resulting in on-the-job struggles, conflicts, complaints, or poor performance. If a colleague notices a YA 
on-the-job struggles, conflicts, complaints, or poor performance. If a colleague notices a YA peer provider struggling on the job and relays this information to a supervisor, the supervisor should follow up with this as soon as possible. Sometimes, YA peers may start to experience symptoms while working and not know how to cope with them in their new role. They may feel ashamed because they perceive they made a mistake on the job and disengage. It is important to outreach to young adult peer providers if they are calling out of work and/or not showing up for work. Supervisors should meet the YA peer where they are at, identify the problem, and partner with him/her to trouble-shoot the situation and discuss potential accommodations (chapter 7).

- Supervision Is Not Therapy. If young adult peer providers consistently bring personal struggles to supervision, supervisors should encourage and support young adult peer providers to seek appropriate mental health support and/or treatment. However, supervisors do not want to prevent the sharing of ANY personal life experiences - they are how we connect as humans in the workplace! It is healthy to talk about hobbies, interests, and even personal struggles with coworkers at times. And an inherent part of the supervisor's role is to support YA peer providers in reframing their life experiences, in order to better engage and motivate young adult clients; talking about past and current life experiences is important. However, if the majority of supervision is spent on processing the YA peer provider's current personal life struggles, instead of focusing on how to use these experiences to increase client support and engagement, the supervisor needs to help the YA peer to understand that supervision is not the place for this and increase the structure of supervision meetings.

- Embrace the Process of On-the-Job Skill Development. Many young adult peers will enter their jobs without experience in managing complex projects and tasks. YA peers need support in organizing tasks, including managing their case load and designing interactive activities. YA peers will likely need coaching about how to articulate their ideas in team meetings. Service note documentation in particular may initially require extra structure and support, including templates with example phrasing and more senior YA peers to advise them on documentation.

- Provide Specificity and Context for New Task Assignments. TAY do not have much work (or school) experience and are often just learning workplace culture and terminology. When faced with an unfamiliar assignment, some TAY do not have the tools (often learned in school) to organize themselves toward developing a plan to engage the assignment. In such cases YA peers may freeze and not complete the work or provide something you're not looking for. For these assignments, be very specific in Guidance for New Task Assignment : - Put in writing

- Offer specifics

- Define terms

- Include examples for formatting, style and length

- Schedule a time to discuss shortly after assignment is made and/or send a follow-up email to support task completion. terms of what you expect (e.g., length of report), the steps required to complete the task, and the due-date. For example, a supervisor might ask a YA peer to write a "report", and report back" to him/her with "recommendations" on how to proceed. This kind of assignment raises questions for the YA peer about the 
necessary depth and comprehensiveness of the report, and the need to cite evidence of facts and research. Thus, assignment specifics might include: sources to consult, approximate length of the report, expected format, time line and due date, and sometimes specific steps to complete the task. Providing them with examples of what you expect is very useful. After a few such assignments, the YA peer will be fine without such specific guidance.

- Effective YA Peer Supervision Must be Reflective. Reflective supervision is an approach used in early childhood and family practice. Reflective supervision aims to increase empathy, integration of multiple perspectives, and practitioner self-awareness. The process of reflective supervision is an exercise in examining parallel processes between family member interactions, family-practitioner interactions, and practitioner-supervision interactions. The supervisor supports the practitioner in reflecting upon their role with clients, including exploring the practitioner's actions, thoughts, feelings, attitudes, and reactions in their work with clients. The process of reflective supervision focuses on the dynamics of relationships within client family systems, between peers and supervisors, and the systems these relationships exist in.

Building strong bonds with YA clients is a key task of YA peers. It provides the opportunity to be reflective about the qualities of these relationships, the impact they are having on YA peers, and how relationships must change in order to achieve desired outcomes. The Illinois Children's Mental Health Partnership has a fantastic Reflective Supervision Guide with resources (https://www.icmhp.org/wp-content/uploads/2020/03/Reflective-Practice-Guide.pdt)

- Additional Peer Supervision Resources:

o Peers in Action: http://peer.hdwg.org/sites/default/files/PACT-SupervisionTools.pdf Building Blocks to Peer Program Success: http://peer.hdwg.org/sites/default/files/6. SupervisingPeersComplete.pdf

o SAMHSA Peer Support Whole Health \& Wellness: http://www.integration.samhsa.gov/ Supervisor Guide to Peer Support Whole Health and Wellness -c- 2013.pdf

\section{Essential Topics}

YA peer supervision must be multidimensional:

1. Performance. Supervision must incorporate "tasked-based" or administrative elements. When addressing on-the-job performance, always begin with identifying what is going well; discuss organization and time management; and any barriers to performing job duties. Use the $\mathrm{SMART}^{1}$ framework to develop goals for annual performance reviews. Brainstorm ideas for addressing workplace challenges together. Encourage YA peers to advocate for clients and to speak up in meetings. Review any accommodations (chapter 7). This task- based element can be conducted by a supervisor who does not provide clinical supervision.

\footnotetext{
A plan that is: Specific, Measurable, Attainable, Relevant, and Timely. See https://en.wikipedia.org/ wiki/SMART_criteria.
} 
2. Skill Development. Supervision must also integrate skill-based training, coaching, and reinforcement. Supervisors should support YA peers in taking inventory of their skills, as well as identifying skills that they can develop to increase client engagement. Setting aside one supervision meeting a month to focus on developing specific skills (such as motivational interviewing) is helpful. A supervisor can also use this time to share opportunities where YA peer providers can continue developing current skills or attain new ones (e.g., upcoming conferences and trainings).

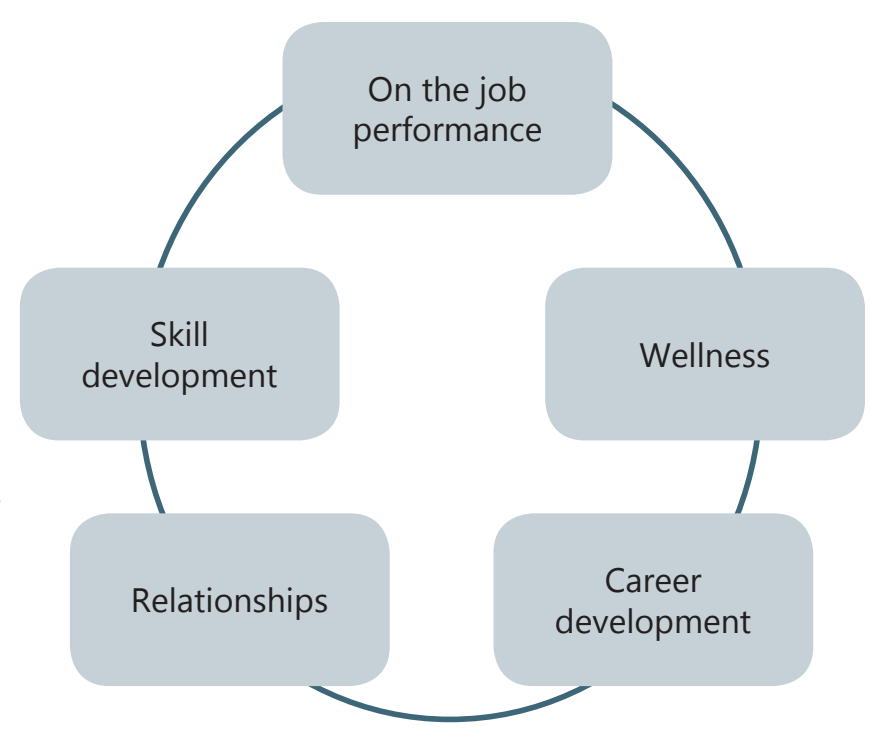

Most often, YA peers need support in developing intra-office communication skills. Many YA peers will use brief and immediate modes of communication through social media messaging platforms (e.g., Facebook) and texting, rather than sending detailed emails or making phone calls and leaving voice mail messages. Supervisors are instrumental in supporting YA peers in communicating with their older co-workers professionally and in the ways that meet agency guidelines. Supervisors can do this by developing context-specific communication practices with email, phone calls, and in-person exchanges. For written communications the Memo format provides that context (template at Appendix D). But written communications are often insufficient when there is disagreement or conflict, and in this case supervisors will need to encourage verbal support, often in-person exchanges. In addition, YA peers may need to be reminded of professionalism in the workplace - and that looking at one's smartphone to check social media feeds must be minimized during work hours.

3. On-the-Job Relationships. Supervision must also support the development of healthy YA peer relationships with youth and young clients, peer and non-peer coworkers, and supervisors. The supervision should review how relationships in the workplace are progressing, highlight positive relational experiences, discuss healthy relational boundaries, review social media policies, and model appropriate relational boundaries.

Managing YA peer-YA client relational boundaries gets tricky in small towns and neighborhoods, because YA peer and client social networks may naturally overlap. YA peers and supervisors must discuss the potential for community run-ins or off-hours client interactions. YA peers must directly ask their clients how they prefer to describe their relationship when these run-ins happen. Supervisors must systematically remind YA peers about confidentiality. Discussing off-hours interactions is necessary to support healthy YA peer relationships with their clients; YA peers will need coaching and support in maintaining healthy boundaries, especially with clients they are likely to interact with regularly outside of work. 
4. Wellness. Supervision must support YA peers in building on-the-job resilience through engaging in self-care, processing challenging workplace situations, and providing resources for maintaining health and wellness on the job. When personal mental health issues and/ or an inability to cope with on-the-job stress continually gets in the way of job performance, supervisors should work with the peer to develop an effective accommodation, and/or support him/her to identify an appropriate treatment program, often through referral to the agency's Employee Assistance Program. For more information see chapter 4.

5. Vocational Development. Supervision must support YA peer career development. The YA peer role cannot be held forever. YA peers are also in the midst of their own vocational development - an experience that they can share in real-time with their clients. Supervisors must support YA peers in exploring and developing their own career plans, support any additional education engagement (including university, college, and/or certificate programs), and support vocational next-steps in relation to skill development and opportunities for future peer and non-peer roles at the agency or beyond. Most importantly, supervision meetings should review the practice of healthy, professional employment and relationship termination.

\section{The Importance of the Young Adult Peer-Supervisor Working Alliance}

Peer support is based on the premise that the possession of lived experience with a serious mental health condition (SMHC) is the foundation for a strong working alliance. ${ }^{\text {lxii }}$ The working alliance is a collaboration based on the development of an attachment bond and a shared commitment to goals and tasks. ${ }^{\text {lxiii }}$ Stronger working alliances are associated with better outcomes and are found to be stronger predictors of outcomes than a therapeutic approach.

The goal of the YA peer supervisor is to be a coach, mentor, and sounding board for YA peer innovation and on-the-job struggles. Supervisors must build a strong working alliance with their YA peers in order for YA peer supervision to be effective.

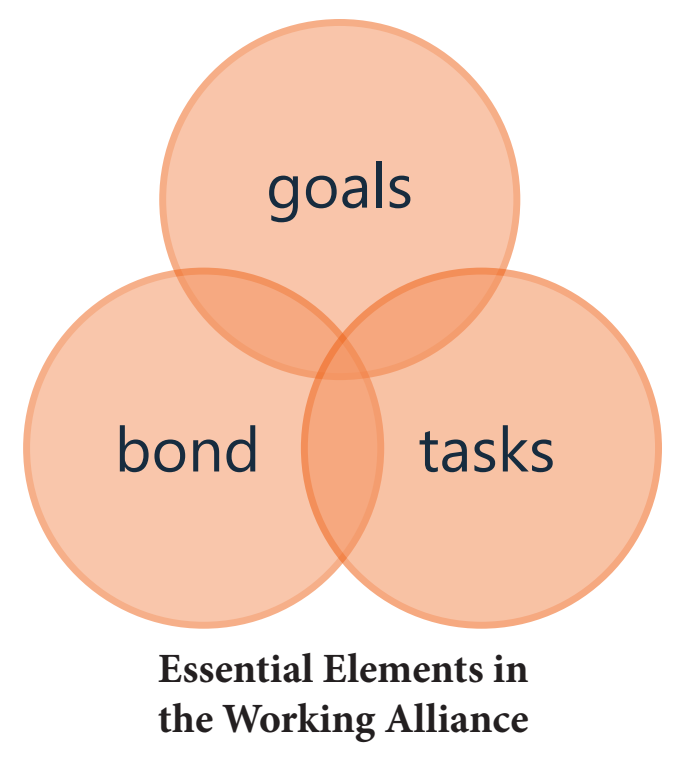

Strong working alliances between YA peers and supervisors:

- Increase the likelihood that a YA peer will discuss any on-the-job struggles with their supervisor, thus allowing for opportunities to assess, develop solutions, and discuss accommodations.

- Increase the likelihood that YA peers will feel comfortable discussing the pros and cons of supervisor suggestions and directives. 
- Model relationship building skills with YA peers on how to work with YA clients. In order to build strong YA peer-supervisor working alliances, supervisors must engage in the following practices:

- Make transparent their personal desire to support YA peers in their work and development. Supervisors must be genuinely motivated in their role as a coach to keep YA peers engaged, challenged, and feeling supported.

- Express intention from day one, to support YA peers in building a foundation of skills that will benefit them far beyond their current role - and that career development and self-care will consistently be part of supervision.

- Be present when meeting with YA peers. Do not check emails and text messages during formal or informal supervision meetings. Put a sign on the door to ensure the meeting is not disrupted.

- Practice mutuality by sharing personal early employment experiences, as well as what you do to stay healthy on-the-job. Be honest. Practice doing what you expect YA peers to do on a daily basis. Recognize that mutuality is challenging, and role model healthy boundaries.

- Be accessible during the workday. When unavailable, provide names of colleagues that YA peers can connect with. This ensures a feeling of on-the-job support among YA peers.

- Recognize that not all YA peer-supervisor relationships result in strong working alliances. Support YA peers in connecting with a colleague with whom they can form a strong working alliance.

- Balance directives with open-ended discussion in supervision. Supervisor directives are key at times when agency policies directly dictate staff action (e.g., in the case of client confidentiality), and processing situations is important in supporting YA peer development. Open-ended questions include:

o How should you respond/intervene? Why? How will the client likely respond to this approach: best-case scenario response vs. worst-case scenario response?

o How would a more senior YA peer or other colleague respond/intervene? Why would they take that approach?

o What are the pros and cons of responding in the way that you proposed?

By asking these process questions in supervision rather than providing answers, YA peers develop problem solving skills and confidence in their role.

Validation followed by solution-focused planning is key in supporting the on-the-job success of YA peers. Supervisors must continue to normalize how working with vulnerable populations can impact worker wellbeing and share their personal stories of on-the-job resiliency. Supervisors can share how they have coped (well and not so well) with challenging work situations, how they acclimated to shifting job tasks and roles, and how they practice self- care on-the-job - all of which can aide in developing a strong working alliance with YA peers.

Following validation and sharing is solution-focused planning. Supervisors and YA peers must partner to develop solutions to challenging work situations. The agreed upon plans should have clear action steps for both the YA peer and the supervisor. By focusing on solutions after validation YA peers experience a mutual sense of agency in generating change, which will serve to strengthen your working alliance. 


\section{Bolstering Young Adult Peer On-the-Job Resiliency}

All YA peers need support and coaching around how to maintain their own mental health and wellness while working. Individual and group clinical supervision provide excellent forums for processing on-the-job challenges. Supervisors should remain open to hearing about the perceived impact that the YA peer role has on YA peer's mental health and wellness. This is especially important, since remaining well at work may have little to do with the YA peer's capacity to manage stress and remain resilient. It may be agency-related issues that are contributing to YA peer health and wellness deterioration (see chapters 3,8 ).

On-the-job resiliency for YA peers can be achieved through the fostering of "psychological capital". Psychological capital is a positive psychological state of mind often characterized by a person's optimism, self-efficacy, optimism, perseverance, hopefulness and resilience. ${ }^{\text {lxxiv }}$ It generates motivation to succeed, accomplish goals, and overcome barriers. One can be diagnosed with a SMHC and even be struggling with mental health symptoms, but also possess selfconfidence and stress management skills to perform their peer job duties. Supervisors play a key role in supporting the development of psychological capital in YA peers. To boost YA peer psychological capital, the agency must embrace a culture of health and wellness, with a range of options to prevent and address work-related stress. For more information see chapter 4.

In addition, supervisors can:

- Role model and practice self-care at work and in supervision;

- Recognize small successes systematically;

- Remind peers that we are all learning through doing our job tasks and will occasionally have off-days where we are not performing at 100\%; and

- Ensure that YA peers utilize paid time off, including personal, vacation, and sick days.

YA peers are especially at risk for compassion fatigue, vicarious trauma, and burnout. There are two ways to prevent these negative work experiences through supervision:

1. Help YA peers set reasonable personal expectations for their personal capacity at work and the for impact that they desire to have on program clients; and,

2. Support YA peers in creating on-the-job self-care plans. Regularly discuss self-care plans and personal self-care practice during supervision.

There are many self-care resources available online for the helping professionals. One excellent resource is: https://socialwork.buffalo.edu/resources/self-care-starter-kit.html.

In order to prevent burnout and feelings of not being effective, YA peers need to understand that their job efforts have a purpose and are having an impact, even if it is not quite the impact a YA peer is personally envisioning for clients. For YA peers to directly observe the impact of their work with youth and young adult clients, it is beneficial to engage in exercises where they 
concretely plan their intervention and then evaluate its impact on client. The following activity is simple and based on the Plan-Do-Study-Act (PDSA) Worksheet (Appendix E).

First, in order to plan the intervention, the supervisor and YA peer must specify the following:

- Activity Goal: Individualized client goal that the YA peer is working with the client on;

- Activity Description: What the YA peer and client do together in order to make progress on the goal (include details like where, when, who, and how);

- Goal Achievement Indicators: How a YA peer and client will know that the activity was successful in making progress or achieving the goal (ideally defined in client's own words).

Then, the YA peer implements their activity with a client. In their next supervision meeting, the YA peer will discuss and document. For many mental health practitioners, this exercise may seem juvenile. However, the process of truly planning actions and evaluating them can be incredibly educational and empowering for YA peers who are new to service planning and delivery.

- Reality Check: What actually happened when the activity occurred? (What was the quality of the activity and the client's response to the activity?)

- Goal Achievement: Describe the measured results based on the planned goal achievement indicators.

- Next Activity: Based on what was learned through the activity delivery, experience, and impact, what will the next planned activity with the client be?

\section{Training, Supervision, and Support for Young Adult Peer SUPERVISORS}

Supervisors have a unique role where they are overseeing and mentoring YA peers, many of whom will be new to this type of professional role. YA peers may not be prepared for the nature of a role that demands connecting with and motivating YA clients through self-disclosure. The YA peer supervisor role should not be taken lightly - and those who are hired/appointed to this role must recognize the complexity of the role and be ready to address stigma, crisis, and trauma on a daily basis. Also, the YA peer supervisor may experience vicarious trauma during the process of developing their stories for strategic storytelling. It is important that agency administrations are aware of the unique role that YA peer supervisors have, as both champions of YA peers in the workplace, and as individuals who support YA peer on-the-job success through reflective supervision and coaching.

YA peer supervisors benefit from regular individual and/or group supervision - especially the opportunity to personally process their on-the-job struggles with supervising YA peers. If supervision for YA peer supervisors is not offered formally, supervisors must seek out mentorship from an individual with experience supervising peers. Also, YA peer supervisors benefit from 
connecting with one another at state and national conferences, which can result in mutually beneficial relationships for processing YA peer supervisory experiences.

Agencies must provide training and on-going support for YA peer supervisors. Currently, there are few trainings available for supervising the work of YA peers; however, Dr. Klodnick has developed such a training for the Massachusetts Department of Mental Health, which can be found here and here.

\section{Moving ON}

YA peers will not stay young adults forever. It is important that organizations focus on the development of YA peers, so that they can eventually move into adult peer/non-peer roles within the same organization, or transition to employment at a new organization or in a different field. Providing opportunities for former YA peers to network (for example, through annual reunions, conferences, or trainings) is a fantastic way for them to remain connected to a community where they are valued greatly. Individual supervisions should systematically include planning for future employment positions and review the practice of healthy, professional employment and relationship termination. 


\section{7 | Addressing Significant Job Difficulties By EFFECTIVELY Negotiating Reasonable ACCOMMODATIONS}




\section{7 | ADDRESSING SIGNIFICANT JOB DIFFICULTIES BY EFFECTIVELY NEGOTIATING REASONABLE ACCOMMODATIONS}

\section{INTRODUCTION}

It is not uncommon for YA peers to have job difficulties due to intrapersonal disruptions, the peer role not being well-defined, lack of workplace support, and/or a negative workplace culture. The evidence shows that creating a system to address significant mental health-related job performance difficulties shortly after they arise greatly improves job performance and retention of employees, including transition age youth. ${ }^{1 \times x v}$ When these kinds of job difficulties are not addressed in a timely fashion, productivity decreases and stress, and absenteeism increase. ${ }^{\text {lxxi }}$

Fortunately, the ADA and related discrimination laws have placed an affirmative duty upon employers to help qualified employees with disabilities to perform the job's essential functions. That is, a YA peer ${ }^{2}$ having job difficulties can consider asking for reasonable accommodations.

Reasonable Accommodations (RA) are modifications or adjustments to ordinary business operations that enable a "qualified" individual with a disability to:

- successfully perform essential job functions;

- enjoy equal employment benefits, privileges and opportunities; and

- apply for a job.

Overall, the evidence shows that RAs for people with SMHC enhance productivity when employees understand the process for requesting accommodations and supervisors are knowledgeable about assessing requests and providing them. ${ }^{\text {lxxvii }}$ Nevertheless, human resources personnel and workplace supervisors are often not enthusiastic about dealing with RA requests or working with an employee to identify and implement the best accommodation. This is largely due to not understanding their RA responsibilities, as well as being concerned about unsubstantiated

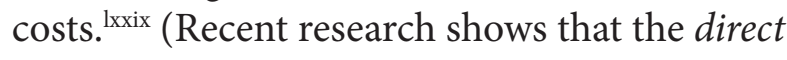

Common accommodations for people with psychiatric disabilities include:

- Flexible scheduling in order to attend counseling or support meetings

- Enhanced job training and coaching

- Training and coaching for co-workers on diversity and stigma

- More intensive individualized supervision

- Provide praise and positive reinforcement

- Divide large assignments into smaller tasks and steps

- Reduce distractions in the workplace

o Moving workplace to quieter area

o Provide space enclosures or a private office

- Unpaid leave

\footnotetext{
${ }^{2}$ As discussed in chapter 4 , "peers" are generally considered as having a "disability".
} 
costs associated with RAs for people with SMHC are often nominal, though there can be indirect costs, such as payments for additional coaching or training.)

Because of the broader productivity implications on providing assistance to people with mental health impairments, We recommend that employers apply this RA mandate not only when there is a legal requirement, but also when the job difficulty has to do with a non-essential job function or is related to a health impairment that does not rise to the level of "disability."

The questions we address in this chapter regarding YA peers are:

1. What are the employer's RA responsibilities?

2. How do supervisors et al. work with the peer to identify the best accommodation?

3. How do they become knowledgeable and practiced regarding their responsibilities?

\section{RA Mandate Basics}

There are two primary phases of employer responsibilities with regard to the RA mandate:

1. How to take action. Employer Notice;

2. When to take action

Reasonable Accommodation (RA) framework

\section{Employer Notice}

The employer's responsibility to consider providing a RA comes into play when:

- An employee states or otherwise indicates that s/he is having trouble with an essential job function that may be related to a disability or illness. Notices do not have to be in writing, use any official language, or even ask for accommodation. Valid requests typically happen when an employee:

o Reports a difficulty with an essential job function, and it relates to his/her health impairment;

o Requests an accommodation because of an impairment is impacting job performance.

- It is "otherwise obvious" to the employer that an employee is having trouble with the job due to an impairment. For example: An employer knows about an employee's psychiatric condition from previous experiences/communications with the employee. If the employer becomes aware that the employee is having trouble with essential job functions possibly related to his/her impairment, that employer is effectively put on notice. 


\section{Reasonable Accommodation Framework}

When put on such notice, an employer must engage in an "interactive process" with the employee with the aim of reaching a resolution on the most appropriate RA(s) (see next section- "Employer-employee interactive dialogue").

\section{Threshold standards to assess the legitimacy of a proposed accommodation} (i.e., whether it meets threshold standards)

1. Is it reasonable?

2. Will it cause "undue hardship" (UH) on business operations?

The factors addressing these two questions are discussed in Appendix F, and specifically regarding the YA peer and undue hardship in the box below.

\section{Assessing Undue Hardship and the YA peer:}

- Accommodation costs are usually indirect:

o YA peer supervisor providing additional supervision;

o When a peer reduces weekly hours and takes leave, other roles on the team may need to be restructured.

- Direct financial costs are not common; they are usually payments for external training or support (e.g., class fees or job coaches) and for replacement staff when the peer takes a leave of absence.

- The YA peer provider can be difficult to replace because of the unique qualities and skills necessary for the role and the limited number of YA peers on staff. Thus "leave" for a YA peer is more likely to present undue hardship issues than for other employees, at least from an operational perspective.

- It is the company's obligation to make every effort to cover the YA peer's hours.

\section{Additional resources on the Reasonable Accommodation mandate include:}

- Appendices F \& G of this toolkit

- Federal Guidances:

o Job Accommodation Network (JAN) is a free service offered by the U.S. Department of Labor's Office of Disability Employment Policy and the leading source of guidance in workplace accommodations. Phone consultation is available to both employers and employees. JAN's website offers a variety of learning materials on RAs.

o There are 10 Americans with Disabilities Act (ADA) regional centers that cover the United States, each responsible for technical assistance and research, including trainings.

o The United States Equal Opportunity Commission also has good background information on the ADA. 
In the remainder of this Chapter we introduce a systemic approach to address job difficulties of TAY employees and provide them with specific RAs and other direct assistance. The four critical cornerstones of this approach are in the box below.

\section{Critical cornerstones for providing RAs and addressing significant job difficulties}

1. There are clearly described protocols for employees to request accommodations or other assistance for disability-related job difficulties;

2. Employers and employees use appropriate interactive tools and processes to discuss RA solutions;

3. RA responsibilities are clearly defined and understood by the employer and staff;

4. Supervisors have the willingness and support to address accommodation issues.

\section{THERE ARE CLEARLY DESCRIBED PROTOCOLS FOR EMPLOYEES TO}

\section{REQUEST ACCOMMODATIONS OR OTHER ASSISTANCE FOR DISABILITY- RELATED JOB DIFFICULTIES}

As noted earlier, many people with SMCH are reluctant to ask for a RA or other assistance. Employees with SMHC are more likely to disclose RA needs and disability status to employers who express enthusiasm for supporting their success (otherwise, disclosure may be seen as risking their job status). ${ }^{1 \times x x}$ This starts as early as the job interview process, in which interviewers express the value the organization places on the use of RAs. This expression of RA value should continue into the workplace, through clear signs/posters, orientations, trainings, as part of the personnel handbook, on the company website, and as expressed through senior staff.

Employees are also more likely to disclose when there is a clear and structured RA request process with a good deal of flexibility.

We thus suggest that organizations:

- Offer a structured form for requesting RAs. As discussed in the next section, a good form can help the requestor provide the employer with the most important information needed to take action. Of note, an employee does not legally need to use the organization's form to make a request- it could be done verbally or using any kind of written note.

- Recommend that employees make their RA requests directly to their supervisors, with the option of approaching other staff when that makes more sense (e.g., the issue directly concerns the supervisor). 
- Post an RA policy statement for all to see in the appropriate format that includes:

o The employer's obligation and desire to accommodate;

o Principles for providing appropriate accommodation (e.g., individualized, respecting the dignity of the worker, maximizing autonomy and integration);

o How to request an RA or related job assistance;

o How the request will be processed;

o The responsibilities of both the employee and employer in this interactive process (see next section);

o A time frame for assessing and deciding on the request;

o Procedures for maintaining confidentiality of health-related information in accordance with applicable privacy legislation. ${ }^{1}$

A very good example of a RA policy statement is the University of Washington RA policy statement.

\section{Employers and employees use appropriate interactive tools and processes to discuss reasonable accommodation solutions}

The Personalized Accommodation ${ }^{\varpi}$ (PA) process is a methodologically sound approach for employers and employees to develop a shared understanding of the job challenges facing an employee with SMHC as well as the supports that will promote his/her success. A fourcomponent framework to direct the PA process is described below:

\section{Four- component framework to direct the PA process}

1. The list of questions to be considered in order by both employees and supervisors

2. Independent preparation and information gathering

3. Employer-employee interactive dialogue

4. Develop a RA and evaluate it

${ }_{1}$ Sometimes, a significant concern for the employee might be whether anyone else in the workplace will be told about the accommodation or the disability. According to the ADA and other laws, no co-worker should be told about the diagnosis or the type of disability. Only co- workers who have oversight and responsibility for RA implementation may know though they are not permitted to share unless necessary. 
Personal Accommodations ${ }^{\oplus}$ process for addressing job

difficulties and developing RAs

\section{Questions to be addressed in order}

Worksheets

1. What are the job's primary functions/tasks?

A

2. What primary functions/tasks the peer is having difficulty with?

A

3. What are the challenges and barriers to successful execution of the functions/tasks?

A

4. What [type(s) of] accommodations might help the peer to effectively perform these functions/tasks?

A, B

5. What is the most effective and reasonable accommodation(s)?

B

II. Independent preparation by employee and supervisor:

- review of questions and

- information gathering

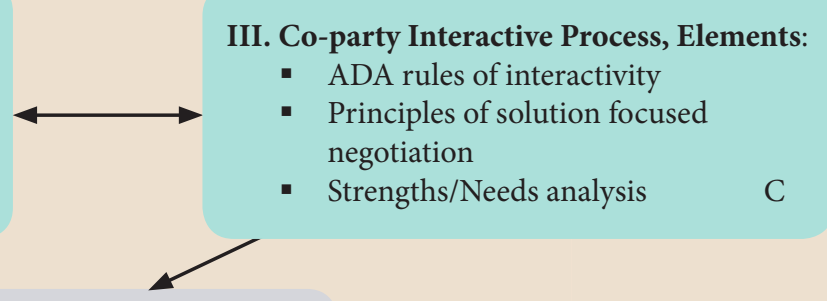

IV. Establish RA

- Implement RA

- Monitor and change as needed

- Evaluate process

The process is generated by a guiding set of consecutive questions and interactive worksheets to provide collective focus, transparency, and accelerated resolutions of issues.

When the employer adopts the PA framework, it should be shared, including the worksheets and advisory tools, with all staff. When employees and employers are working from the same accommodation framework, there is a level of transparency that generates clarity for both parties.

\begin{tabular}{|c|c|}
\hline \multicolumn{2}{|c|}{ Worksheet } \\
\hline A & TheJobChallengesandAccommodations Worksheet \\
\hline B & The Accommodations Analysis Worksheet \\
\hline $\mathrm{C}$ & Strengths/Needs Worksheet \\
\hline
\end{tabular}

\section{Questions to be addressed in order}

The employer and employee will use the 5 questions listed below and other tools herein 
to deliberate individually and together towards selecting an RA(s). The five questions are designed to be addressed in the order presented when preparing independently and as a framework for employer- employee discussions. The questions to be addressed and worksheets associated are:

Questions

1. What are the job's primary functions/tasks

2. What primary functions/tasks is the peer is having difficulty with?

3. What are the challenges and barriers to successful execution of these functions/tasks?

4. What [type(s) of] accommodations might help the peer to effectively perform these functions/tasks?

5. What is the most effective and reasonable accommodation(s)? $\underline{\text { Worksheet }}$

A

A

A

A, B

B

The questions are ordered as such because initial consideration and discussion should not focus on the nature of the person's disability or diagnosis, but instead on identifying the difficulties the employee is having with primary job functions. When a discussion starts with "disability," it becomes very easy for the parties to stay on that topic rather than addressing the perhaps more challenging aspects of improving job performance. In addition, when using labels/diagnoses in such a discussion, misunderstandings can arise from terminology that is ambiguous and/or understood differently by the parties.

In addition, peer supervisors have reported that when discussing a YA peer's mental health problems with him/her, they are tempted to act as (and can feel like) therapists or crisis managers for that person. This approach takes an emotional toll on supervisors who begin to feel responsible for the person's overall wellbeing, and it creates a relationship more similar to psychologist/patient than supervisor/employee. It also does not address the functional concerns that will reduce tension in the existing relationship. Thus, the question format initially pulls the supervisor away from those roles and narrows the focus toward holistic problem-solving that takes into consideration workplace factors, such as staff training needs. (As part of that there may be accommodations related to the person's healthcare or just general advice, but it's not the initial focus.)

The consecutive nature of the questions does not mean that each one needs to be addressed fully and completely before moving to the next one. For example, it may be difficult to provide a complete answer for question 3 until discussing with the other party, but that shouldn't stop each party from "penciling in" some possible accommodations in question 4.

The Job Challenges and Accommodations Worksheet [A] is the worksheet people can use to answer questions 1-4 initially as they go through the process. The Accommodations Analysis worksheet [B] guides the parties toward evaluating and selecting proposed RAs (questions 4 and 5). These worksheets provide a framework for identifying valid/legitimate accommodations, assessing their relative utility, and agreeing on the best one. 


\section{Independent preparation and information gathering}

The Precision Accommodation ${ }^{\circledR}$ process starts when an employee with SMHC or an employer/ supervisor believes that there are difficulties related to that employee's job performance. The first step for this initiating party then is to go through at least the first 4 of the 5 consecutive questions using the Job Challenges and Accommodations Worksheet [A]. For the employee, this will result in a coherent narrative identifying these job difficulties and the kinds of accommodations that might help. An employer/supervisor will also have a coherent narrative to present to an employee. Completing these questions will allow them to present to the other party:

[Employee] "I am having difficulties with job functions probably because Here are some job changes I think could address them and make me more productive [Supervisor] "I believe you are having difficulties with___ job function[s]..... here are some ideas for addressing them ___ "

Independent preparation by the employee and employer is very important in framing the concern holistically, including consideration of workplace culture and behavior of other staff. In responding to these questions each party should consider obtaining advice from people they know or experts in needed areas:

\section{Supervisor}

o Staff, consultants, or colleagues with knowledge of the kinds of difficulties the employee is having, such as learning difficulties, anxiety, poor communications; EAP staff can be useful here (chapter 4);

o Staff with expertise in providing RAs, including knowledge of costs and success rates of RAs under consideration;

o Other staff with knowledge of the employee's performance. Employee:

o Health care professionals who treat the employee;

o Case managers and other people who provide support;

o Independent health/disability assessment professionals;

o Friends and family;

o Trusted co-workers, current or past;

o Mentors.

\section{Employer-employee interactive process}

It is a legal requirement that employers and employees engage in an interactive process toward reaching the best RA decision. There are two main reasons: First, both parties have important information to offer. Second, employee involvement builds trust, and increases the likelihood that s/he will make a strong effort to make the RA work.

The element of the interactive process presents a structure for engaging in productive discussions towards agreeing to the best RA. It may take only a single discussion to reach a resolution, or there could be more complex issues that require a more extended discussion and negotiation. The 
primary procedural elements of an interactive process here are:

- Principles of Interactive Dialogue for RA discussions

- Principles of Negotiation and Communications

\section{Principles of Interactive Dialogue for RA discussions}

- The employer should acknowledge receipt of an employee's RA request immediately. The supervisor should meet with the employee as soon as possible, or if not available, delegate the task;

- The initial discussion should ALWAYS begin with the employee's job tasks and functions. Do not start by discussing illness, etc.;

- As people go through each question, they should stop and ask for the other party's perspective and/or affirmation;

- The employer must engage in a "flexible give-and-take," including:

o Being active in seeking information as needed from the employee or designees (e.g., doctors);

o If rejecting an RA proposal, communicate the specific reasons to the employee, which allows the employee to make a reasonable counter-proposal;

- An ongoing discussion is necessary because issues are not always fully addressed in a single session. Parties need time to step away from meetings, think on their own (questions 1-5), seek out additional information, and gain outside advice;

- The employer should not, in any way, condition the granting of an RA on the employee's promise of improved performance, nor require the employee to take unpaid medical leave until the employer has time to consider at-work accommodations. 


\section{Principles of Negotiation RA Discussions and Communications}

At any point of discussion there may be feelings or disagreement, but these basic rules will help ameliorate those emotions and refocus parties on the issues at hand.

\section{Common techniques include:}

- Focus on interests [productivity, wellness], not positions

o Explain and reiterate your interests clearly.

o Ask the other party to describe his/her interests.

o Discuss these interests to identify common goals.

- Separate the people from the problem:

o Rather than resolve conflict, solve the problem.

o Think of each other as partners in negotiation rather than as adversaries.

o Avoid blaming or attacking the other person.

o Speak primarily about yourself or your opinions. Use "I" statements, such as "I feel" or "I think."

o Acknowledge your emotions to the other party.

o Try not to react emotionally to another's emotional outbursts.

o Symbolic gestures such as apologies or expressions of sympathy can help to defuse strong emotions.

- While talking, stay focused on what you are trying to communicate:

- Actively listen to the other party (with your full attention), occasionally summarizing the speaker's points to confirm your understanding without conveying judgment.

- Have a pad of paper present to take notes and clarify the relevant matters you are trying to communicate to the other party.

- Use experts agreed to by both parties to inform discussion. Types:

o Topical, e.g., guidance with a specific disability

o Facilitator of discussions

\section{Utilizing the worksheets}

Now the parties can use Worksheets A and B as their personal guide for presenting information, and leave it at that. They can also share with the other party their responses/narrative on paper or electronically. (We have found the third question on reasons for difficulties to be the most sensitive to others, so the parties may agree to not share that part.) Keep in mind that it should be the peer's choice of whether and to what extent they share their Worksheet A. We have seen that as the process goes along, employees are more likely to share their worksheet with the employer.

Worksheet A can be used more interactively by using a web-based "shared documents" interface in which different authors can work on a shared document together, and by uploading and downloading supporting reports, such as doctor's records and estimated costs of an accommodation. With this level of transparency there is greater potential for issues to be addressed more quickly.

Because each party likely possesses incomplete information for most questions (particularly 
3-5, e.g., employee - nature of the employee's impairment; employer - the company's available resources for particular accommodations), each must be prepared to readjust his/her responses to a questions. Regardless of attempts to follow this question set approach, negotiation may stall and the parties may start to feel emotional and less focused on the problem. In this case we recommend the use of the Strengths/Needs Worksheet $C$, which in our experience can redirect participants to thoughtful discussion and resolution.

\section{Develop RA and Evaluate It}

Below we outline the process for developing and evaluating the chosen RA. It is important to record the RA agreement and an implementation plan in writing, including objectives and goals, actions taken to date, and a timeline for implementation and re-evaluation. Since each employee is unique, there may be some trial and error in developing the accommodation.

\section{The RA plan should be revised if:}

- The RA is not producing the desired results;

- The RA is overly burdensome (costs/time);

- There are obvious ways to improve the RA; and/or

- The employee prefers a legitimate alternative.

Employment culture and policies have a major impact on the capacity to identify and implement the best RA. After providing a number of RAs, the employer should evaluate the factors that have supported and/or impeded successful implementation. Information can be gathered from employees with disabilities, other employees, and supervisors. Major questions for this analysis include:

- Do company policies and practices need to be revised?

- How does workplace culture impact the success of employees with disabilities, including RA implementation?

o How can culture be impacted to improve working conditions for people with disabilities? [See chapter 4 of this toolkit.]

\section{RA RESPONSIBILITIES ARE CLEARLY DEFINED AND UNDERSTOOD BY THE EMPLOYER AND STAFF}

RA responsibilities apply not only to management and supervisors, but also to employees in terms of

- $\quad$ Providing the needed information;

- $\quad$ Engaging in interactive dialogue;

- Implementing the RA and making modifications as needed.

Resources on the RA mandate (above) all provide useful training materials, with several specific to people with mental health conditions. In addition, the ADA regional centers provide various levels of training for employers (above). Nevertheless, it is vital for the organization to produce a robust RA training for all employees, using effective training methods discussed in chapter 8 . In 
addition, supervisors should be able to access organizational RA specialists (often residing in HR) who can advise on sticky issues.

\section{Supervisors Have the Willingness and Support to Address ACCOMMODATION ISSUES}

Staff can be trained and knowledgeable, but to carry out their RA responsibilities they will need organizational support and a willingness to engage. That is, supervisors in particular will need to have:

- An open mind and heart, valuing the perspectives and contributions of people with diverse backgrounds and cultures, with the assistance of company diversity initiatives as described in chapter 4;

- The support of higher level and HR staff to attend to their own practical and emotional needs while making headway toward addressing employment issues of people with disabilities (see chapter 8);

- Performance evaluations that assess in part their effectiveness in managing job difficulties for people with SMHC, YA peers in particular, and RA requests (chapter 9). 


\section{8 | Preparing and Engaging Non-Peer Staff}




\section{8|PREPARING AND ENGAGING NON- PEER STAFF}

When an organization hires YA peers without educating non-peer staff on the impact on their own jobs, confusion and conflict among staff can ensue. And without the support of nonpeer staff, YA peers will have difficulty carrying out their responsibilities. Specific trainings and teamwork exercises are very important toward building the strong working relationships necessary to support YA peer job success.

\section{Training and Support for Non Peer Staff}

Upon starting a job as provider staff, the new employee will presumably receive an orientation to the organization, regular trainings thereafter, and supervisory reinforcement on key topics. A significant part of those educational efforts should be devoted to developing staff capacity to work with and support YA peers.

The most critical part of training introduces and delineates the role of the young adult peer to non-peer staff. Chapters 1, 2 \& 3 of this toolkit contain basic information on this role, including helpful content that can be used for training purposes:

\begin{tabular}{|lc|}
\hline \multicolumn{1}{|c|}{ Training component } & Chapter \\
Unique characteristics and value & 1 \\
CPS code of ethics & 1 \\
Positive impacts of introducing YA peer role & 2 \\
\hline
\end{tabular}

And in the table below, we offer a more specific presentation on how YA peers can address client struggles and challenges directly.

\begin{tabular}{|c|l|l|}
\hline \multicolumn{2}{|c|}{ Specific Benefits of Peer Support for TAY Clients } \\
\hline Client Struggle & Unique Aspects of Peer Role & Provides \\
\hline Feeling hopeless & $\begin{array}{c}\text { Using aspects of personal } \\
\text { recovery story }\end{array}$ & Inspiration \\
\hline Lonely and devalued & Mutuality, shared experiences & Acceptance, feeling valued \\
\hline
\end{tabular}




\begin{tabular}{|c|c|c|}
\hline $\begin{array}{c}\text { Inertia: confusion about } \\
\text { how to move forward }\end{array}$ & $\begin{array}{c}\text { Using recovery story, } \\
\text { role modeling }\end{array}$ & $\begin{array}{c}\text { Initiation of recovery } \\
\text { plan, wellness and social } \\
\text { life tips }\end{array}$ \\
\hline $\begin{array}{c}\text { Voice not present or } \\
\text { ignored [e.g., in treatment } \\
\text { decision making] }\end{array}$ & $\begin{array}{c}\text { Advocating on behalf } \\
\text { of the client, and role } \\
\text { modeling on how to do it }\end{array}$ & Sense of empowerment \\
\hline
\end{tabular}

In addition, the Meaningful Roles for Peer Providers in Integrated Healthcare manual developed by the California Association of Social Rehabilitation Agencies (CASRA) provides useful information and tools, including:

- "Staff concerns self-assessment," which helps to identify staff training needs (page 98);

- Responses to staff on their concerns about the peer role (page 99);

- Discussion questions for staff and peers (page 107).

YouthMove also offers valuable materials youth peer support training.

The introduction of the YA peer role into a treatment team inherently alters the job duties of non-peer staff (as discussed in chapter two). Therefore training for non-peer staff should be made available: 1) making referrals to a YA peer, 2) effective communications with YA peers ("Interacting with Peers" below), and 3) supporting YA peers to be active participants in the treatment team and other meetings (chapter 9). More experienced staff can offer those trainings, or workshops.

Beyond a basic understanding of the YA peer role, there are also important topical areas to cover in staff training. This includes the necessary organizational culture elements to supporting YA peers on-the-job success contained in chapter 3. In this chapter, we further explore aspects of necessary organizational culture elements and make recommendations on how to make trainings most effective.

If not already present, non-peer staff should be trained in the following areas (with associated links):

- Person-first language, ${ }^{\text {lxxxi }}$

- The myths of mental illness, ${ }^{\text {lxxxii }}$ and

- Person centered services (see Person Centered Care \& Planning: Policy to Practice to Evaluation). ${ }^{\text {lxxxiii }}$

\section{Interacting with Peers}

Training should address the tendency of some staff to ask YA peers direct personal questions related to their diagnosed mental health conditions. Staff must be taught that although YA peers were hired in part because of their lived mental health experience, they are not open books for sharing those experiences, however well-intentioned those questions are.

Organizations must train and support non-peer staff to relate to peers as they might to other staff. For young adults in particular, personal questions about a difficult past can be painful, 
stigmatizing, and marginalizing. This is particularly true of specific personal questions such as "Where have you been hospitalized," and "What meds have worked best for you?" As one young adult peer provider explained:

"More than a few times, staff have asked me about my experience with my illness and recovery. I give them a general overview, but they are really interested in specific information such as meds I've been on and how I got along with my family. This is annoying and condescending. I don't ask them specifics of their health background or personal life. But if they ask for advice I feel better about sharing personal stuff...

Some better questions [for non-peer staff to ask peer staff] are:

'Why is it so hard for clients to engage in treatment?'

'Do you know about xx hospital and what's it like?'

With these I can use aspects of my personal life if it makes sense. I get to choose what to share to maintain my personal wellness and privacy while giving the information they really need. If they want to know me better, the best way is just to hang out and talk."

Non-peer staff must also be thoughtful about when and how to ask young adult peer providers more general personal questions, such as "can you tell me what it's been like for you go through treatment and recovery?" These questions are more likely to be well received when the staff person and young adult peer already have a good relationship and/or when tied to an administrative or clinical initiative, not asked just out of curiosity. Examples of such requests or questions include:

"My client is struggling with severe depression. Is there anything from your lived experience you could use to help him move forward? How would you use it?"

"Many clients here don't want to even talk about medications. Can you tell me more about why that's the case? How do young people experience psychiatric medications? Are there young adults who find medications helpful?"

"We'd like you to lead or co-lead a workshop for staff on recovery. It would be most effective if you could share aspects of your lived experience to illustrate points."

\section{Key components of Effective Trainings for Non-Peer Staff}

We recommend that trainings be delivered according to the following principles:

- Young adult peers take an active role in developing and presenting the training. Trainings that are led or co-led by peers can change other's attitudes about people with serious mental health conditions (SMHC). In fact, these trainings are most effective when people with SMHCs share their recovery stories. ${ }^{\text {lxxiv }}$

- Trainings are offered on a regular basis, with materials available via agency intranet.

- Trainings integrate active learning methods (e.g., discussion; games), as opposed to a heavy reliance on passive, lecture-based methods and videos. Active learning is much more likely to improve employees' cognition and motivation to address workplace issues, prejudice in particular. Other active learning approaches include simulation exercises, case studies, group discussion, and collaborative work projects.

- With regard to trainings aimed at addressing employees' prejudice and stigma, Nemec et al. (2015) advise against using "role plays" because trainees tend to rely on stereotypes when asked to portray people with SMHC. ${ }^{\text {lxxv }}$ Instead, they recommend the use of "Real-Plays", 
in which trainees use struggles they experience in their portrayals of people with SMHC. For example, participants can incorporate a personal experience of being judged based on a stereotype and relate feelings of frustration and helplessness.

According to Nemec et al., (2015, pp. 204):

"Teaching and training settings can be structured to convey a message of hope and recovery, rather than on focusing on the person served in negative terms, as belligerent, resistant, unmotivated, or disinterested. Trainers should carefully construct "real-play" experiences rather than "role plays." A real-play exercise has the trainee in the "client" seat, describing his or her own life situation, personal values, strengths, goals, and challenges. In contrast, in role plays, the trainee pretends to be a "real client," often portrayed in a pejorative manner as resistant, unmotivated, and challenging. This scenario has the trainee imagining and acting out stereotypes rather than experiencing a real-life human experience.

By contrast, in well-designed real-play situations, the trainee discovers that many "problems" or "issues" experienced by people served parallel to what they, themselves, have experienced. For example, in real plays used in training Wellness Coaches, trainees can learn valuable lessons, such as the difficulty of changing habits, that listing personal strengths can be a challenge, and that hearing advice often creates an active effort to argue against the suggestions offered. Through real-play practice, trainees come to see these difficulties and challenges as common human nature issues, creating empathy for the people they serve. A real play can offer a transformative learning experience... that creates disruption, such as facing a contradiction in what the learner "knows" to be true."

"Real-Play" example with regard to YA Peers:

Remember a time as an adult in which you were employed or in school and felt poorly understood and even mistreated by the employer, other staff, teacher, or other students. Now think about:

- How it made you feel? What kind of effort did you think it would take to succeed in your role?

- How did those feelings affect your thoughts and actions, both at work/school and in your personal lives?

- How could your employer/school have done to make your situation better?

\section{Team Building Strategies}

Team building strategies are designed to build trust and relationships among staff; develop shared understandings of staff roles; and establish clear channels of communication between peer and non-peer staff. An excellent Compendium of Team Building Tools has been developed at University of California-Berkeley. lxxvi

There are several effective approaches to team building, but one of the most effective is the colearning process. Co-learning fosters the reciprocal exchange of knowledge, skills, and capacity among teammates, recognizing that everyone has something to contribute that collectively 
strengthens the team. More specifically, team members can conduct workshops for the group on their areas of expertise, such as YA peers on the experience of having a SMHC and staff on best and evidence based practices. ${ }^{\text {lxxxvii }}$ Staff can also conduct workshops on the topics discussed above, demonstrating their personhood and value to the team. This approach is remarkable in breaking down barriers through relaxed discussion on different and conflicting views. ${ }^{\text {lxxxviii }}$ Cross-training, in which the various professionals train their colleagues on their specific professional roles, skills and expertise, is also very effective in breaking down barriers.

Another good team building approach is for YA peers and non-peers to work together on a project without tight time frames. The project could be focused on quality improvement, developing a service component, or preparing a grant application. For example, a project could be drawn from an actual small grant funding announcement in which there are multiple opportunities to apply over a series of months or years. Here, peers and non-peers can share diverse perspectives, discuss those differences, and come to resolution on what to include in the proposal. Conflict that may arise will highlight areas where more training for staff and peers is needed.

In addition, organizations can create opportunities for informal interactions between peer and non-peer staff, such as sponsoring and paying for group lunches and social outings. Employee mentorship for peers is also an effective approach to facilitate peer education and socialization. ${ }^{\text {.xxxix }}$ If the mentorship relationship between YA peers and their colleagues develops naturally - that is most desirable. However, assigning a more senior peer staff member to mentor a YA peer may also be beneficial. 


\section{9 | INFRASTRUCTURE AND FrAMEWORK}




\section{9|INFRASTRUCTURE AND FRAMEWORK}

An organization's culture and capacity to support YA peer success is dependent on its infrastructure, including the policies and procedures that determine how work roles and responsibilities are assigned, carried out, and coordinated. We have identified five aspects of organizational infrastructure not specifically addressed in other chapters that will require direct attention in order to effectively integrate the young adult peer role into your organization. As described below, these aspects serve as mechanisms for organizational change and learning.

\section{Organizational Infrastructure and Framework Domains}

- Committed and strong leadership;

- Strong organizational communications that endorse the young adult peer role;

- Connecting \& working with human resources (HR);

- Effective staff hiring \& accountability practices;

- Young adult peers influencing policy and practice.

\section{Committed And Strong Leadership}

Employees take their attitudinal cues from organizational leadership and their supervisors, particularly around the prioritization of goals and tasks. Thus, establishing an inclusive culture that promotes successful employment for young adults diagnosed with SMHC begins with organizational leadership, particularly the $\mathrm{CEO}$ and project directors, but also with other high-level clinical and administrative executives and the board of directors. Leadership can demonstrate its commitment to the young adult With regard to YA role development, leadership must:

- Project hope and optimism;

- Role model good practice;

- Communicate about implementation challenges and the plan to address them;

- Find and assign resources supporting implementation. peer role by:

- Projecting Hope and Optimism. Leaders demonstrate commitment by extolling the virtues of the YA peer role. Organization leaders show their enthusiasm by sharing stories of:

o The direct contributions of agency YA peers to improvements in client outcomes and overall quality of agency services;

o Former or current agency clients who have become YA peers and are successfully impacting the lives of their clients. 
Leaders motivate employees by projecting optimism and hope in both the tone and content of their communication to their agency. Ambivalent communication about YA peers will often weaken the leader's credibility in building a case for why change is needed within the organization to integrate the YA peer role.

- Role Modeling. Organizational leaders serve as role models by meeting with YA peers and their supervisors to express their appreciation for their efforts, and troubleshoot system- and agency-based challenges as they arise. They also visibly bring YA peers into decision-making about the young adult service design. A photograph of YA peer training graduates standing strong with elected or appointed officials and other agency leadership is a strong endorsement of the role.

Leaders who get behind a concept or innovation, such as YA peers, before it is a mainstream TAY treatment model component demonstrate the highest credibility with key stakeholders. These early adopters often take a public stand and are well positioned to develop that role moving forward by working with young adult peers.

- Communicating About Implementation Challenges and the Plan to Address Them.

Successful leaders provide relevant and truthful information about implementation challenges and how they will be addressed. Thus, leadership can and should openly:

o Recognize that the introduction of the YA peer role may challenge staff perspectives on treatment approaches;

o Provide education and training necessary for non-peer staff to understand the YA peer role, as discussed in chapter 8 ;

O Specify to non-peer staff that their job duties now also include: 1) understanding the YA peer role, 2) connecting clients to YA peers as indicated, 3) respectfully communicating and coordinating services with YA peers, and 4) supporting the inclusion of the YA peer voice in team meetings;

o Reiterate that non-peer staff jobs are not at risk, though staff will be evaluated in part by their efforts and progress toward supporting the YA peer initiative;

o Support constructive dialogue among YA peers and other staff by providing team building tools and strategies (chapter 8).

Leaders should also address employees' questions, generate creative ideas, and prioritize ideas to address any lack of motivation to champion and/or support the YA peer role. One useful tool is cross-training, with both YA peers and non-peer staff (e.g., team leaders, social workers, psychiatrists) to educate each other on their respective professions and job responsibilities.

- Finding and Assigning Resources to the Young Adult Peer Role. Funding and staff time should be available to support the best-practices described in this manual. Resources can be obtained through state contracts and grants for transition-age youth program and policy development. Local foundation grants can been used to strengthen the YA Peer role in a program. Any proposed budget should include sufficient and ongoing training for staff, supervisors, and YA peers on matters described in this Toolkit. 


\section{Strong Organizational Communications that Endorse the Young Adult Peer Role}

Organizational communication, or "messaging," is the transmission of key information related to an agency's mission, culture, practice, and strategic initiatives to employees and stakeholders. For employers, messaging is designed to engage employees and stakeholders in supporting a new or challenging initiative by extolling its virtues and negating unsubstantiated concerns. These messages can now be disseminated widely through social media, including CEO Blogs, Twitter, Facebook, LinkedIn, and Instagram.

Messages endorsing the YA peer role can fully or even partially make the business case for the role's integration (chapter 2). This includes explaining how the YA peer role will:

- Improve services and outcomes;

- Benefit non-peer staff;

- Enhance the potential for organizational development and growth;

- Increase diversity in the workplace;

- Increase service enrollment of YA clients and their families.

Messaging that endorses the YA Peer role is especially important to counteract long-held stereotypes of young adults diagnosed with mental health conditions. Organizational leaders should share stories with staff that describe YA peers as capable, confident, and collaborative. These stories often describe how young adults diagnosed with SMHC came to be peers, and how they enhance the team's functioning and positively influence client outcomes. First person accounts of employers, peers, and clients can powerfully illustrate this message. Pictures, narratives, and articles depicting young adult peers as successful (e.g., acting as mentors or volunteers helping others) are also valuable.

Organizational message statements are meant to be influential, both externally and internally.

Internal messages are designed to influence and direct staff priorities and activities. Internal messaging strategies target managers, supervisors, and co-workers, and should be designed to foster awareness, acceptance, and support of the young adult peer role. Important internal messaging strategies should include:

- The identification and empowerment of champions among management staff who actively promote, encourage, and guide the implementation of the YA Peer role.

- Trainers and speakers who can speak from direct experience:

o YA peers who share specifics about their success as peers and their own recovery,

o Management and supervisors who have overseen or otherwise played a role in implementing a YA Peer initiative. Many of the best trainers and organizational champions are former detractors who have become converts;

- Stories and statements in internal publications, such as employee newsletters and brochures; 
- Written stories and posters physically placed on office walls and in corridors;

- The very presence of peers in the workplace is itself the strongest message. Peers have been trained to tell their story and explain their role, and can use those skills with other employees. Peer influence will be stronger when there are more of them and in positions of higher authority (see below).

External messages are meant to influence a wider audience, including external stakeholders such as funders, government agencies, community members, consumers, family members, and potential employees. Primary goals of external messaging are to develop alliances, recruit and hire, and obtain funding. For example, the Young Adult Vocational Program of Eliot Community Human Services in Massachusetts regularly distributes newsletters that present perspectives of both peer mentors and program clients. In addition, The Massachusetts Department of Mental Health (DMH) supports various consumer groups, including the statewide Transformation Center (which operates the certified peer specialist training in Massachusetts), to sponsor a young adult driven website, called Speaking of Hope, which generates many of these messages.

\section{An organization's primary messages are most broadly expressed through its mission} statement, associated list of objectives, and other communications of organizational "values." These statements should state, reflect, and support the organization's dedication to YA peer providers as key to the overall service approach. Mission statements most visibly offer a window into organizational purpose and direction. They also offer insight into the language used by senior managers in communicating the organization's service approach. Mission statements with a tight focus and activist tone are associated with better organizational performance. For providers that serve primarily transition-age youth, here is a sample mission statement:

"Believing that all transition-age youth can recover and grow, the staff of this organization, through a cooperative effort with the community, will ensure a safe, caring environment, the availability of Young Adult peer providers, and vocational and social opportunities that support clients in becoming contributing members of society and leading personally fulfilling lives."

Providers that serve multiple populations, including transition-age youth, may not have the opportunity to be as specific in their mission statement about the value of YA peers. However, mission statements can include important value statements, for example:

- Peer providers greatly enhance the quality of our services while improving outcomes;

- Young adult services include a focus on vocational and career development.

Mission statements can also include useful objectives, such as: "We aim to employ [N] young adult peer mentor(s) on each treatment team, who will be respected and integrated members of the teams."

In addition, messages are often embedded in key organizational planning and policy documents, including policy and practice change statements that embrace the integration of the YA peer role. For example, an organization can affirm its support for young adult peers by stating in a published organizational report or white paper: "We are committed to growing peer employment opportunities for young adults and will take affirmative steps to employ, retain, and advance young 
people in our organization." Another example: "As we continue to expand our young adult services, we realize that peer support and empowerment are essential to our business strategy. We are committed to creating an environment in which young adult peers are valued and respected."

\section{Connecting and Working with Human Resources Departments}

Human Resources (HR) departments generate agency policies and procedures regarding personnel management (e.g., hiring, retention, and termination), company-wide wellness initiatives, non-discrimination and diversity efforts, benefits management, and trainings. The impact of HR activity on staffing, organizational culture, and practice implementation is very relevant to the integration of YA peers.

Previous toolkit chapters discussed multiple areas in which HR plays an important role: ADA and reasonable accommodations, wellness initiatives, compensation, hiring and evaluation standards, and trainings. Here, we discuss issues that typically arise when program developers and HR do not have preliminary discussions about the YA peer role, including: peer job classification, qualifications and pay, pre-screening in hiring, organizational policies, and benefits.

\section{HR responsibilities for YA peer implementation include:}

- Peer job classification, qualification, \& pay;

- Pre-hiring screening and questions;

- Changes in HR and other organizational policies;

- Benefits counseling

Because many YA peers have often had limited employment experience, HR will at times need to be more deliberate and patient in educating YA peers about the organization and its personnel policies. HR can offer group presentations that allow for questions, but should also provide opportunities for individualized staff consultation.

\section{Peer Job Classification, Qualifications, \& Pay}

As discussed in chapter 5, we have found that HR departments usually have not been involved in the development of YA peer job duties or the overall job description, and are often the last to know about this new, unique position before hiring takes place. In these cases, HR is not ready with the appropriate classification(s) for the peer job, which can hold up hiring. This occurs most frequently in larger organizations that have many HR protocols and practices that necessitate multiple levels of approval.

HR's early involvement in shaping the YA peer role can create the conditions for the efficient hiring of YA peers and the identification of any agency policies/practices that could impede this process. When program leadership includes HR in the discussion about the need for a "peer" job classification in advance of hiring peers, the potential for creating that classification is greatly enhanced. In fact, union contracts often allow for the creation of new job types, but time for 
sharing information and negotiation is needed. Regardless, HR staff, program developers, and unions will need to maintain a flexible perspective as they continue their discussions. When helping to develop a YA peer job description, HR must:

- Recognize that common requirements of direct service jobs can be less relevant to the YA peer job than other qualifications, such as lived experience and ability to tell one's recovery story. In order to recruit the best YA peers, typical requirements of direct service workers may need to be loosened, such as educational qualifications (chapter 5).

- Take care to not automatically screen out or otherwise diminish the overall qualifications of YA peer job applicants with criminal records and/or incomplete education degrees, particularly in light of the job's essential duties and responsibilities (chapter 5).

- If there is no "peer" job description yet approved, work with program leadership (and unions if relevant) to link to or create the appropriate job classification based on the essential job functions.

- If a new "peer" job class is created, establish a series of job grades based on the job's requisite essential responsibilities and qualifications, and toward promoting competitive hiring, providing fair compensation, offering training/educational opportunities, and determining clear promotion pathways. Identifying a clear path for promotion should reflect management's view that these employment opportunities are not viewed as deadend jobs.

- Ensure that salary and benefits reflect the duties and skills required for the particular YA peer role, both as a matter of fairness and in order to attract high quality YA peer candidates.

- Clearly outline responsibilities, performance standards, salary range, and paths for promotion in YA peer job descriptions.

\section{Pre-Hiring Screening \& Questions}

Best practices regarding peer job interview questions and screenings were discussed in chapter 5 with regard to three common qualification factors:

- Disability

- Criminal Record

- Education

To summarize, the basic best practice rules regarding YA peer applicants are:

- Consider the above factors as job criteria only when directly relevant to the peer job's duties and performance expectations;

- Ask for information about these factors only when directly related to the essential job duties and performance expectations, and narrowly so;

- When criminal convictions are relevant, ask about them later in the process, and certainly not at the initial interview (except with regard to disqualifying criteria). The idea is to provide all eligible applicants with a fair chance to initially present themselves on equal footing with other applicants, and to not discourage them from further pursuing the position. 


\section{Changes in Human Resource \& Other Organizational Policies}

It is not uncommon for agencies to have HR policies in place that have not been written in a way to fully support the hiring of qualified YA peers. Human Resources should work with program staff to identify policies that are likely to impede the recruitment and retention of YA peers and make the necessary changes. Several important examples of how to recognize potentially detrimental policies for the hiring and on-the-job success of YA peers are discussed below:

\section{Language}

While employing YA peers, an agency should look and listen for any terminology or other style of language that stereotypes or stigmatizes people diagnosed with $\mathrm{SMCH}$, including clients and community members. The presence of stigmatizing language in policies can be very discouraging for YA peers. It is better to use language that communicates the value of "lived experience" of mental health difficulties and recovery:

\section{Stigmatizing language in organizational policies}

\begin{tabular}{|l|l|}
\hline Stigmatizing Language in Policies & Non-Stigmatizing Language in Policies \\
\hline $\begin{array}{l}\text { "The mentally ill" } \\
\text { (Non-person first) }\end{array}$ & $\begin{array}{l}\text { "Person diagnosed with...; "Person with the } \\
\text { lived experience of..." }\end{array}$ \\
\hline $\begin{array}{l}\text { "Severe and persistent mental illness" } \\
\text { (Not recovery oriented) }\end{array}$ & $\begin{array}{l}\text { "Severe mental illness"; "Serious mental health } \\
\text { condition which the person has experienced for } \\
\text { years" }\end{array}$ \\
\hline $\begin{array}{l}\text { "Non-compliant" } \\
\text { (Judgmental/Blaming) }\end{array}$ & "Not participating in treatment because__ \\
\hline $\begin{array}{l}\text { "Lacks insight into his/her mental... } \\
\text { "(Judgmental/Unclear) }\end{array}$ & $\begin{array}{l}\text { "The client disagrees with the clinical } \\
\text { assessment. More specifically..." }\end{array}$ \\
\hline
\end{tabular}

Health and wellness improvement options should ideally be universal to all staff Normalizing individualized health and wellness support for staff will improve overall productivity and morale, and negate the impression that people with health issues are given special job advantages. Benefitsf or all staff regardless of their health status could include access to "reasonable accommodations," including fl exibility in work schedule and time off beyond the coverage of the Family and Medical Leave Act (FMLA). In addition, agency policy can emphasize clinical supervision that is regular, individualized, and supportive. For more information see https://drc.arizona.edu/workplace/inclusive-workplace-practices.

\section{Dual Relationships}

Provider agencies are rightly concerned about dual or overlapping relationships between practitioners and clients that could impair the practitioner's focus on the preferences and best interests of the client. In this regard, some agencies have prohibited the hiring of current or former clients as provider staff based on the heightened possibility of their having existing 
romantic or other personal relationships with current clients. We recommend against such a broad prohibition because former or current clients can work at YA programs where they have not been clients. (And when such a prohibition is not applicable to non-mental health clients, it inherently institutionalizes prejudicial practices.) A better approach is to use the agency's dual relationship conflict policies that are applied to all job applicants.

Some agencies have prohibited former clients from working as provider staff at the program from which they have received services. One reason is that provider staff may be uncomfortable having former program clients as colleagues, particularly those to whom they have provided direct case management or counseling. In addition, the possibility of relational conflicts with current clients is raised here, though mitigated by the length of time since the job applicant was a program client.

We recommend against blanket prohibitions of former program clients working as peer staff. First, there can be tremendous benefits to clients working with former clients. Short of having a "conflicting" relationship, such former clients tend to have a strong sense of a real shared lived experience that fosters validation and a sense of kinship. Former program clients can be the best guide for current clients in navigating a complex treatment and vocational system of support. Most importantly, former clients in the role of YA peer are the most direct evidence to transitionage youth clients (and their families) that growth and recovery (using this program) are in reach.

Additionally, there may be a limited number of successful YA peer candidates available to work with a program's clients, particularly so in rural areas. Thus, excluding former clients from working as YA peers without an individualized conflicts assessment is essentially a denial of access. There may be instances in which having a client or former client as a peer is not a good idea, but we suggest that this be assessed on a case-by-case basis according to agency policy.

In addition, we recommend that agencies explore the ethical and practical implications of hiring former clients. Agency leadership should support discussions among staff to play out some scenarios and fully understand risk in relation to client benefits. It may make sense to bring in a therapeutic ethics expert to lead discussions towards applying existing ethical standards (organizational and peer professional) toward hiring former clients. It also makes sense to work with clinicians to establish comfort levels for working as fellow employees with former clients. Involving Human Resources in these discussions will help ensure they are comfortable with the outcome.

An agency's dual relationship policies should be clearly communicated to all staff.

\section{Benefits Counseling}

Providing a livable wage and benefits is essential to creating a thriving YA peer workforce. Some YA peers receive government benefits, such as Supplemental Security Income (SSI), and their taking a paid peer position will often impact these benefits. HR should incorporate benefits counseling into its HR orientation policies in order to promote job opportunities 
for and retention of YA peers. Th is counseling can provide important information to help a peer maintain benefitsw hile working, and can assist in developing strategies for getting off benefi ts smoothly. Thisinc ludes using the Social Security Administration's Trial Work period and the extended period of eligibility, and taking advantage of the Ticket to Work program, which connects people with disabilities to free vocational supports. For more information, see SAMHSA's SOAR-WORKS project.

Agencies should also review their health insurance policies to see how they will aff ect YA peers who are hired, particularly those who are part-time employees. In Aff ordable Care Act Medicaid Expansion States, most peers have been able to access health insurance as they transition to employment, but in non-expansion states, health insurance coverage gaps continue to be present. Nevertheless, the majority of states have a Medicaid Buy-in provision, which allows people with disabilities whose earnings and resources might otherwise make them ineligible to purchase Medicaid coverage while working (as of 2015, 46 states had the buy-in provision). If an employer fi nds that YA peers are unable to access health insurance that will cover the care that is supporting their recovery, they may want to lower their health insurance eligibility standards (e.g., hours worked per week) to be more inclusive. For more information, see Connecting Youth and Young Adults to the Affordable Care Act from YouthMove (2014).

\section{Effective Staff Hiring and Accountability Practices}

For YA peers to succeed in the workplace, staff will need to be understanding and supportive; this is particularly true for staffw ho work on treatment teams with YA peers. But as noted earlier, some staff are not supportive of YA peers, and may even undermine the peers' effo rts. Thus it is important to hire, evaluate, and hold accountable staff who possess the capacity and willingness to support the YA peer role. Important criteria for staff include:

- Open-Minded, Respectful, \& Compassionate

o Empathetic \& excellent at listening;

o Interested in different and contrasting perspectives, for example on a variety of traditional and non-traditional approaches to treating SMHC;

o Not paternalistic, understands and accepts dignity of risk, \& empowerment;

- Capacity to Motivate \& Support Client Development

o Possesses motivational skills;

o Cares about own development;

o Demonstrates patience in developing client's vocational skills, especially when teaching skills for the first time;

- Embraces the Notion of Mental Health Recovery

o Believes that people can and do recover from SMHCs;

o Understands the myths and prejudices that face individuals with SMHCs;

- Understands \& Values the Peer Provider Role

o Understands the role and its benefits;

o Willingness to take on the challenges to integrating peer providers;

o Excitement, on a personal level, to partner with YA peers; 


\section{- Understands \& Values Young Adult Life \& Culture}

o Understands that young adults have a unique culture;

o Understands how and why young adults have different needs from older adults.

Hiring qualified staff is not enough for ensuring performance, particularly for tasks and responsibilities not typically taught in school. Performance evaluations and accountability of staff working with YA peers are also important. It is necessary for companies to hold employees accountable for their performance by evaluating and taking action on staff performance in light of agreed-upon performance standards or targets. Accountability strategies often include the following elements:

- Process and/or outcomes assessments for individual staff members, not just their program overall;

- Clear responsibilities for the staff's supervisor regarding performance evaluation;

- Feedback on performance that is direct and timely;

- Clear expectations for the impact of performance evaluations, including performance improvement plans, recognition and opportunities regarding positive evaluation, and negative actions regarding continual poor evaluations.

\section{Young Adult Peers Influencing Policy and Practice}

A key role, and perhaps the most difficult one, of the YA peer is to influence the treatment team and organizational culture and practice. There are several institutional barriers that inhibit YA peers from actively participating in and influencing organizational decisions. First is the stigma of having a diagnosis and second is the stigma of youth. "Adultism," or the attitude that young people have little or nothing of value to offer to older adults, can be found in many organizations. As a result, the input of YA peers might be stymied or ignored because of stereotypes associated with age and/or having a mental health diagnosis. Fortunately, there are proven approaches for YA peers to actively participate in organizational decisions.

Young adult peers can have the most influence on organizational planning when they:

- Make up a significant portion of the service provider workforce in relation to other professional groups;

- Are educated on the best \& evidence based practices;

- Are active participants on committees and workgroups relevant to their work;

- Are in an organizational leadership roles.

As noted by Jones in her guidance manual, Peer Involvement and Leadership in Early Intervention in Psychosis Services: From Planning to Peer Support and Evaluation ${ }^{x c i}$ (page 14): 
A "critical mass" of peers... is likely to bolster peer confidence and involvement because any individual member can (in most circumstances) count on "back up" from other peer members. In addition, "critical mass" facilitates projects in which peers are able to check in with someone they feel will understand if they are feeling disempowered or "unheard," or, for example, if a peer felt that a comment during a committee meeting was offensive but is not sure if they are "being oversensitive" or "over-reacting." Sometimes the ability to exchange a nonverbal wink or "knowing look" with a peer across the room during a meeting can make a world of difference.

Young adults are at a disadvantage in any provider policy discussion because they are newer to their work and less familiar with agency practices, acronyms, and organizational terminologies. Therefore, YA peers should be provided with opportunities to attend trainings and workshops on current and best practices and policies related to young adults. Ideally, more experienced staff could mentor and support YA peers to promote their perspective in the organization.

YA peer involvement in agency workgroup and committee discussions about practice and policy involvement will not influence provider decisions unless their participation is active. YA peer involvement is not truly active unless they:

- are involved in workgroups from their inception;

- have a voice during these meetings;

- that this voice is actually heard and valued.

For example, a provider may create a workgroup to address difficulty engaging young adults in treatment. YA peers may find themselves placed in a workgroup with non-peer older staff, whose focus starting off is to address young adult "non-compliance" with medication because of their' "lack of insight" into mental illness. Under the right circumstances, YA peers are likely to challenge the premise of this approach. This would require that the YA peers have received the requisite trainings for their role and YA peers make up a significant number (e.g., one-third) of the group. Ideally they are being mentored by a more experienced employee. Then these YA peers could introduce the idea the medication discontinuation is due to many factors that need to be addressed, including not experiencing benefits, experiencing significant side effects, and/ or being homeless and disorganized. By enhancing the discussion these young adult workgroup participants demonstrate the importance of young adult clients' experience and opinions in contributing to agency improvement efforts.

When considering young adult membership in workgroups, it is also important to consider which YA peer(s) might be the best match for workgroup involvement (i.e., the peer that has the most interest in and experience with the topic). For example, if there's a workgroup on the use of guardians with young adults, it is important to involve a peer who is interested in the issue and perhaps has experience to share on this matter.

A common barrier to active YA participation is that older adults will often talk over YA peers who are less experienced and may be a minority in the group. Our recommendation is that at least one older adult at the meeting be responsible for facilitating the peer's involvement, by creating space for a YA peer who is trying to speak up, and by directing questions to the YA peer. Having 
a facilitator who is adept at ensuring total participation is key. Another best practice is to require that there is more than one YA peer participating in the event.

Dr. Nev Jones, in her guidance manual referenced above offers an excellent chapter [2] on the meaningful involvement of YA peers in program planning, emphasizing asset-based community development, partnerships with peer organizations, and project planning matching.

In addition, Jonathan Delman offers advice on the use of community-based participatory action research methods to involve peers in Young Adults Getting Involved: Participatory Action Research \& Transition Age Youth. ${ }^{\text {xci }}$

Young Adult Integration into Organization Leadership Organizations that employ YA peers benefit from having at least one experienced YA peer in a leadership position within the organization. That YA peer can play a major role in developing young adult related policies and practices, training staff who work with YA peers, and in helping to supervise YA peer staff. Good peer leaders not only advise other staff, but centralize peer education for YA peers. Peer staff leaders can organize quarterly meetings among peer staff to share positive stories around their work, discuss common issues, and provide emotional and practical support. At some agencies, the peer leader also acts in a supervisory capacity, offering administrative supervision and conflict resolution.

A number of provider organizations employ a peer in senior management or as a vice president. This visibility sends a clear message to staff that the role matters, the organization is serious about its implementation, and there is technical assistance available within the organization. For example, Advocates, Inc. in Massachusetts has Vice President of Peer Support and Self-advocacy, who "is responsible for developing and overseeing peer support and self-advocacy for the entire organization, as well as oversight of all human rights mechanisms." In addition, at Thresholds in Chicago, the Director of Recovery oversees client advocacy groups and works as a liaison between client advocacy groups and administration in addition to ensuring that the peer voice is infused in all agency departments.

With regard to provider agencies generally focused on adults, there is unlikely to be more than one peer in an organizational leadership position, which will typically be held by a more experienced older adult. Some thought should be given as to how adult and young adult peers can collaborate to bring forward the young adult voice and lived experience. At the very least, both young adult and older adult peer groups should come together on occasion to discuss common issues. YA peers can work with the older adult peer leader to strategize on improving quality and advocacy with leadership, and to make changes that improve how an agency thinks about, works with, and treats YA peers and clients. YA peers benefit significantly from mentoring opportunities with more seasoned peer provider staff.

In addition, providers should build relationships with peer-run organizations in their locality for technical assistance, guidance on employing peers and to more readily recruit peers. In addition, YA peers introduced to such organizations also benefit by being exposed to examples of successful peer employment, important policy and advocacy issues, and access to peer support 
groups. Below are resources for peer-run groups:

- Adult driven peer policy and advocacy organizations in most of the 50 states (often funded in part by SAMHSA);

- Local Chapters of YouthMove, a national organization led by young adults whose aim is to promote the youth voice in all aspects of mental health decision making. 


\section{References}

i. Substance Abuse and Mental Health Services Administration (SAMHSA), Center for Behavioral Health Statistics and Quality. (2015). Behavioral health trends in the United States: Results from the 2014 National Survey on Drug Use and Health (HHS Publication No. SMA 15-4927, NSDUH Series H-50). Retrieved from https://www.samhsa.gov/data/sites/default/files/NSDUH-FRR1-2014/NSDUH-FRR1-2014.pdf

ii. Courtney, M.E., Dworsky, A., Cusick, G.R., Havlicek, J., Perez, A. \& Keller, T. (2007). Midwest evaluation of the adult functioning of former foster youth: Outcomes at age 21. Chicago, IL: Chapin Hall.

iii. Wagner, M., Newman, L., Cameto, R., Garza, N., \& Levine, P. (2005). After high school: A first look at the post-school experiences of youth with disabilities: A Report from the National Longitudinal Transition Study-2 (NLTS2). Retrieved from: https://remote.umassmed.edu/fulltext/,DanaInfo=files.eric. ed.gov+ED494935.pdf

iv. Millner, U. C., Rogers, E. S., Bloch, P., Costa, W., Pritchett, S., \& Woods, T. (2015). Exploring the work lives of adults with serious mental illness from a vocational psychology perspective. Journal of counseling psychology, 62(4), 642.

v. McGahey, E., Waghorn, G., Lloyd, C., Morrissey, S., \& Williams, P. L. (2014). Formal plan for selfdisclosure enhances supported employment outcomes among young people with severe mental illness. Early intervention in psychiatry.

vi. Bruns, E. J., Walker, J. S., \& Penn, M. (2008). Individualized services in systems of care: The wraparound process. The system of care handbook: Transforming mental health services for children, youth, and families. Baltimore: Brookes.

vii. Clark, H. B., \& Unruh, D. K. (2009). Transition of Youth and Young Adults with Emotional or Behavioral Difficulties: An Evidence-Supported Handbook. Brookes Publishing Company. PO Box 10624, Baltimore, MD 21285.

viii. Blash, L., Chan, K., Chapman, S. (2015). Peer provider workforce in behavioral health: A landscape analysis. Retrieved from: http://healthworkforce.ucsf.edu/sites/healthworkforce.ucsf.edu/files/Report-Peer Provider_Workforce_in_Behavioral_Health-A_Landscape_Analysis.pdf

ix. Blau, G. M., Caldwell, B., Fisher, S. K., Kuppinger, A., Levison-Johnson, J., \& Lieberman, R. (2010). The Building Bridges Initiative: Residential and community-based providers, families, and youth coming together to improve outcomes. Child Welfare, 89(2), 21.

x. Heinssen, R. K., Goldstein, A. B., \& Azrin, S. T. (2014). Evidence-based treatments for first episode psychosis: components of coordinated specialty care. Retrieved from http://gopher.nimh.nih.gov/health/topics/ schizophrenia/raise/nimh-white-paper-csc-for-fep_147096.pdf

xi. Department of Health and Human Services (2015). Informational Bulletin: Coverage of Early Intervention Services for First Episode Psychosis. Rockville, MD. Retrieved from https:// www.medicaid.gov/federal-policy-guidance/downloads/CIB-10-16-2015.pdf

xii. Gopalan, Geetha, Sang Jung Lee, Ryan Harris, Mary C. Acri, and Michelle R. Munson. Utilization of peers in services for youth with emotional and behavioral challenges: A scoping review. Journal of Adolescence 55 (2017): 88-115.

xiii. Blash, L., Chan, K., Chapman, S. (2015). Peer provider workforce in behavioral health: A landscape analysis. Retrieved from: http://healthworkforce.ucsf.edu/sites/healthworkforce.ucsf.edu/files/ReportPeer_Provider_Workforce_in_Behavioral_Health-A_Landscape_Analysis.pdf

xiv. Geenen, S., Powers, L. E., Phillips, L. A., Nelson, M., McKenna, J., Winges-Yanez, N.,Blanchette, L., Croskey, A., Dalton, L.D., Salazar, A., \& Swank, P. (2015). Better Futures: A randomized field test of a model for supporting young people in foster care with mental health challenges to participate in higher education. The Journal of Behavioral Health Services \& Research, 42(2), 150-171.

xv. Chinman, M., George, P., Dougherty, R. H., Daniels, A. S., Ghose, S. S., Swift, A., \& Delphin-Rittmon, M. E. (2014). Peer support services for individuals with serious mental illnesses: Assessing the evidence. Psychiatric Services, 27, 392-401.

xvi. Walker, J.S., \& Gowen, L.K. (2011). Community-based approaches for supporting positive development in youth and young adults with serious mental health conditions. Portland, OR: Research and Training 
Center for Pathways to Positive Futures, Portland State University.

xvii. Radigan, M., Wang, R., Chen, Y., \& Xiang, J. (2014). Youth and caregiver access to peer advocates and satisfaction with mental health services. Community Mental Health Journal, 50(8), 915-921.

xviii. Id.

xix. $\quad$ See Delman, J. \&Klodnick, V.V. (2016). Factors Supporting the Employment of YA peers: Perspectives of Peers and Supervisors. Community Mental Health Journal, 1-12.

xx. Moran, G. S., Russinova, Z., Gidugu, V., \& Gagne, C. (2013). Challenges experienced by paid peer providers in mental health recovery: A qualitative study. Community Mental Health Journal, 49(3), 281-291.

xxi. Klodnick, V. V., Sabella, K., Brenner, C. J., Krzos, I. M., Ellison, M. L., Kaiser, S. M., ... \& Fagan, M. A. (2015). Perspectives of Young Emerging Adults With Serious Mental Health Conditions on Vocational Peer Mentors. Journal of Emotional and Behavioral Disorders, 23(4), 226-237.

xxii. Spencer, R. (2006). Understanding the mentoring process between adolescents and adults. Youth \& Society, 37, 287-315.

xxiii. Id.

xxiv. Delva, D., Jamieson, M., \& Lemieux, M. (2008). Team effectiveness in academic primary health care teams. Journal of Interprofessional Care, 22(6), 598-611.

xxv. Hogan, M. F. (2003). New Freedom Commission report: the President's New Freedom Commission: recommendations to transform mental health care in America. Psychiatric Services, 54(11), 1467-1474.

xxvi. Mead S (2003). Defining Peer Support. Retrieved from https://mhepinc.org/defining-peer-support/.

xxvii. Arnett, J. J. (2001). Conceptions of the transition to adulthood: Perspectives from adolescence through midlife. Journal of Adult Development, 8(2), 133-143

xxviii. Delman, J. (2016). Promoting Young Adult Mental Health through Electronic and Mobile Technologies. Retrieved from https://www.nasmhpd.org/sites/default/files/Assessment\%205_Promoting\% 20Young\%20Adult\%20Mental\%20Health.2.pdf.

xxix. Diomidous, M., Chardalias, K., Magita, A., Koutonias, P., Panagiotopoulou, P., \& Mantas, J. (2016).

Social and psychological effects of the internet use. Acta informatica medica, 24(1), 66.

xxx. Jager, J., Keyes, K. M., \& Schulenberg, J. E. (2015). Historical variation in young adult binge drinking trajectories and its link to historical variation in social roles and minimum legal drinking age.

Developmental psychology, 51(7), 962.

xxxi. Kertesz, S. G. (2016). Turning the tide or riptide? The changing opioid epidemic. Substance abuse.

xxxii. Jager, J., Schulenberg, J. E., O’Malley, P. M., \& Bachman, J. G. (2013). Historical variation in drug use trajectories across the transition to adulthood: the trend toward lower intercepts and steeper, ascending slopes. Development and psychopathology, 25(02), 527-543.

xxxiii. Krupa, T. (2009). Understanding the stigma of mental illness in employment. Work 33:413-425.

xxxiv. Charles, J. L. (2013). Mental health provider-based stigma: Understanding the experience of clients and families. Social Work in Mental Health, 11(4), 360-375.

xxxv. Davidson, L., Harding, C., \& Spaniol, L. J. (Eds.). (2006). Recovery from severe mental illnesses: Research evidence and implications for practice. Center for Psychiatric Rehabilitation, Sargent College of Health and Rehabilitation Sciences, Boston University.

xxxvi. Rosenberg, J., Rosenberg, S., Ellefson, S., \& Corrigan, P. (2015). Public Mental Health Stigma and Mass Shootings. SAJ Forensic Science, 1(1), 1.

xxxvii. Ellison, M. L., Russinova, Z., Lyass, A., \& Rogers, E. S. (2008). Professionals and managers with severe mental illnesses: findings from a national survey. The Journal of nervous and mental disease, 196(3), 179-189.

xxxviii. Delman, J., \& Klodnick, V. V. (2016). Factors Supporting the Employment of Young Adult Peer Providers: Perspectives of Peers and Supervisors. Community mental health journal, 1-12.

xxxix. Couser, G. P. (2008). Challenges and opportunities for preventing depression in the workplace: a review of the evidence supporting workplace factors and interventions. Journal of Occupational and Environmental Medicine, 50(4), 411-427.

xl. Corbière, M., Villotti, P., Lecomte, T., Bond, G. R., Lesage, A., \& Goldner, E. M. (2014). Work 
accommodations and natural supports for maintaining employment. Psychiatric Rehabilitation Journal, 37(2), 90.

xli. MacDonald-Wilson, K. L., Russinova, Z., Rogers, E. S., Lin, C. H., Ferguson, T., Dong, S., \& MacDonald, M. K. (2011). Disclosure of mental health disabilities in the workplace. In I. Z. Schultz \& E. S. Rogers (Eds.), Work Accommodation and Retention in Mental Health (pp. 325-340): Springer New York. xlii. Scheid, T. L. (2005). Stigma as a barrier to employment: mental disability and the Americans with Disabilities Act. International Journal of Law and Psychiatry, 28(6), 670-690

xliii. Brohan, E., Henderson, C., Wheat, K., Malcolm, E., Clement, S., Barley, E. A., \& Thornicroft, G. (2012). Systematic review of beliefs, behaviors and influencing factors associated with disclosure of a mental health problem in the workplace. BMC psychiatry, 12(1), 1.

xliv. Erdos Knapp, D., Faley, R. H., \& Long, L. K. (2006). The Americans with Disabilities Act: A review and synthesis of the legal literature with implications for practitioners. Equal Opportunities International, 25(5), 354-372.

xlv. Brohan, E., Henderson, C., Wheat, K., Malcolm, E., Clement, S., Barley, E. A., \& Thornicroft, G. (2012). Systematic review of beliefs, behaviors and influencing factors associated with disclosure of a mental health problem in the workplace. BMC psychiatry, 12(1), 1.

xlvi. Marquis, J. P., Lim, N., Scott, L. M., Harrell, M. C., \& Kavanagh, J. (2008). Managing diversity in corporate America: An exploratory analysis. Santa Monica, CA: RAND Corporation. http://www.rand.org/ content/dam/rand/pubs/occasional papers/2007/RAND OP206.pdf.

xlvii. Corrigan, P. W., Angell, B., Davidson, L., Marcus, S. C., Salzer, M. S., Kottsieper, P., ... \& Stanhope, V. (2012). From adherence to self-determination: Evolution of a treatment paradigm for people with serious mental illnesses. Psychiatric Services. 63(2), 169-173.

xlviii. Dickey, B., \& Ware, N. C. (2015). Risk Is Not a Four Letter Word: Social Integration and Developmental Growth. American journal of psychiatric rehabilitation, 18(4), 363-376.

xlix. LaPorte, T. M., Haber, M. G., \& Malloy, J. M. (2014). Wraparound Team Composition, Youth Selfdetermination, and Youth Satisfaction in Transition Services. The Journal of Behavioral Health Services \& Research, 1-19.

1. Id.

li. Luthans, F., Youssef, C. M., \& Avolio, B. J. (2007). Psychological capital: Investing and developing positive organizational behavior. In D. Nelson \& C.L. Cooper (Eds.), Positive Organizational Behavior: Accentuating the Positive at Work (pp. 9-24). Thousand Oaks: Sage Publications.

lii. Hollenbeck, K. (2015). Promoting Retention or Reemployment of Workers after a Significant Injury or Illness (No. 99caa302888a4be68d16d276cabec012). Retrieved from https://www.mathematica.org/ourpublications-and-findings/publications/promoting-retention-or-reemployment-of-workers-after-asignificant-injury-or-illness.

liii.Lerner, D., Rodday, A. M., Cohen, J. T., \& Rogers, W. H. (2013). A systematic review of the evidence concerning the economic impact of employee-focused health promotion and wellness programs. Journal of Occupational and Environmental Medicine, 55(2), 209-222.

liv. Richmond, M. K., Pampel, F. C., Wood, R. C., \& Nunes, A. P. (2016). Impact of employee assistance services on depression, anxiety, and risky alcohol use: a quasi-experimental study. Journal of Occupational and Environmental Medicine, 58(7), 641-650.

lv. Hollenbeck, K. (2015). Promoting Retention or Reemployment of Workers after a Significant Injury or Illness (No. 99caa302888a4be68d16d276cabec012). Retrieved from https://www.mathematica-mpr.com/ our-publications-and-findings/publications/promoting-retention-or-reemployment-of-workers-after-asignificant-injury-or-illness.

lvi. Henry, A. D., Laszlo, A., \& Nicholson, J. (2015). What does it take to build an employment collaborative for people with disabilities? Journal of Vocational Rehabilitation, 43(1), 1-15.

lvii. Monaco, K. (2015). Disability insurance plans: trends in employee access and employer costs. Beyond the Numbers: Pay \& Benefits, 4,(4). Retrieved at http://www.bls.gov/opub/btn/volume-4/pdf/disabilityinsurance-plans.pdf.

lviii. Dewa, C. S., Hoch, J. S., Carmen, G., Guscott, R., \& Anderson, C. (2009). Cost, effectiveness, and 
cost-effectiveness of a collaborative mental health care program for people receiving short-term disability benefits for psychiatric disorders. The Canadian Journal of Psychiatry, 54(6), 379-388.

lix. Nieuwenhuijsen, K., Faber, B., Verbeek, J. H., Neumeyer-Gromen, A., Hees, H. L., Verhoeven, A. C., ... \& Bültmann, U. (2014). Interventions to improve return to work in depressed people. Cochrane Database Syst Rev, 12.

lx. Tan, L., Wang, M. J., Modini, M., Joyce, S., Mykletun, A., Christensen, H., \& Harvey, S. B. (2014). Preventing the development of depression at work: A systematic review and meta-analysis of universal interventions in the workplace. BMC Medicine, 12(1), 1-11.

lxi. $\quad$ Volker, D., Zijlstra-Vlasveld, M. C., Anema, J. R., Beekman, A. T., Brouwers, E. P., Emons, W. H., ... \& van der Feltz-Cornelis, C. M. (2015). Effectiveness of a blended web-based intervention on return to work for sick-listed employees with common mental disorders: Results of a cluster randomized controlled trial. Journal of medical Internet research, 17(5).

lxii. Martin, A., Sanderson, K., \& Cocker, F. (2009). Meta-analysis of the effects of health promotion intervention in the workplace on depression and anxiety symptoms. Scandinavian Journal of Work, Environment \& Health, 35(1), 7-18.

lxiii. White, M. I., Dionne, C. E., Wärje, O., Koehoorn, M., Wagner, S. L., Schultz, I. Z., ... \& Hsu, V. (2016). Physical activity and exercise interventions in the workplace impacting work outcomes: A stakeholdercentered best evidence synthesis of systematic reviews. The International Journal of Occupational and Environmental Medicine, 7(2 April), 739-61.

lxiv. Miller, J. J., Grise-Owens, E., Addison, D., Marshall, M., Trabue, D., \& Escobar-Ratliff, L. (2016). Planning an organizational wellness initiative at a multi-state social service agency. Evaluation and program planning, 56, 1-10.

lxv. Pollitz, K., \& Rae, M. (2016) "Workplace Wellness Programs Characteristics and Requirements," The Kaiser Family Foundation. Retrieved at http://kff.org/private-insurance/issue-brief/workplace-wellnessprograms-characteristics-and-requirements/.

lxvi. Salazar, S., Way, V., DeRyke, M., \& Tondora, J. (2014). Developing Policies and Procedures for Employing Peers in the Workplace. Retrieved from https://docs.google.com/viewer? a=v\&pid=sites\&srcid=ZGVmYXVsdGRvbWFpbnxjb2FsaXRpb25wYXJhY2h1dGV8Z3g6NDA4NzBiODQ wZDkxNzMyZg.

lxvii. Gates, L., \& Akabas, S. (2007). Developing strategies to integrate peer providers into the staff of mental health agencies. Administration and Policy in Mental Health and Mental Health Services Research, 34, $293-$ 306.

lxviii. Blash, L., Chan, K., Chapman, S. (2015). Peer Provider Workforce in Behavioral Health: A Landscape Analysis. Retrieved from http://healthworkforce.ucsf.edu/sites/healthworkforce.ucsf.edu/files/ReportPeer_Provider_Workforce_in_Behavioral_Health-A_Landscape_Analysis.pdf.

lxix. Id.

lxx. Delman, J., \& Klodnick, V. V. (2016). Factors Supporting the Employment of Young Adult Peer Providers: Perspectives of Peers and Supervisors. Community Mental Health Journal, 1-12.

lxxi. Daniels, A. S., Tunner, T. P., Powell, I., Fricks, L., \& Ashenden, P. (2015). Pillars of Peer Support VI: Peer Specialist Supervision. Retrieved at www.pillarsofpeersupport.org.

lxxii. Davidson, L., Chinman, M., Sells, D., \& Rowe, M. (2006). Peer support among adults with serious mental illness: a report from the field. Schizophrenia bulletin, 32(3), 443-450.

lxxiii. Bordin, E. S. (1979). The generalizability of the psychoanalytic concept of the working alliance. Psychotherapy: Theory, Research \& Practice, 16(3), 252.

lxxiv. Luthans, F., Youssef, C. M., \& Avolio, B. J. (2007). Psychological capital: Investing and developing positive organizational behavior. In D. Nelson \& C. L. Cooper (Eds.), Positive organizational behavior: Accentuating the positive at work (pp. 9-24). Thousand Oaks, CA: Sage Publications.

lxxv. Whalen, D., Gimm, G., Ireys, H., Gilman, B., \& Croake, S. (2012). Demonstration to maintain independence and employment (DMIE). Final report submitted to the Centers for Medicare \& Medicaid Services. Washington, DC: Mathematica Policy Research. Retrieved from https://www.mathematica.org/ our-publications-and-findings/publications/demonstration-to-maintain-independence-andemployment-dmie 
lxxvi. Pomaki, G., Franche, R. L., Murray, E., Khushrushahi, N., \& Lampinen, T. M. (2012). Workplace-based work disability prevention interventions for workers with common mental health conditions: a review of the literature. Journal of occupational rehabilitation, 22(2), 182-195.

lxxvii. Schultz, I. Z., Milner, R. A., Hanson, D. B., \& Winter, A. (2011). Employer Attitudes Towards Accommodations in Mental Health Disability. In I. Z. Schultz \& E. S. Rogers (Eds.), Work Accommodation and Retention in Mental Health (pp. 325-340): Springer New York.

lxxviii. Kaye, H. S., Jans, L. H., \& Jones, E. C. (2011). "Why Don't Employers Hire and Retain Workers with Disabilities?” Journal of Occupational Rehabilitation, 21(4), 526-536.

lxxix. McDowell, C., \& Fossey, E. (2015). Workplace Accommodations for People with Mental Illness: A Scoping Review. Journal of Occupational Rehabilitation, 25(1), 197-206.

lxxx. Brohan, E., Evans-Lacko, S., Henderson, C., Murray, J., Slade, M., \& Thornicroft, G. (2014). Disclosure of a mental health problem in the employment context: qualitative study of beliefs and experiences. Epidemiology and Psychiatric Sciences, 23(3), 289-300.

lxxxi. Psychiatric Rehabilitation Association (2003). PRA Language Guidelines. Retrieved at https://www. nccdp.org/resources/PRAlanguageguidelines.pdf.

lxxxii. UMDNJ - CSPNJ. Retrieved at http://shp.rutgers.edu/dept/smi/documents/ mythsandmeetingpeoplemodule.pdf.

lxxxiii. Kirk, T., \& Tondora, J. (2007). Person Centered Care \& Planning: Person-Policy to Practice to Evaluation. Retrieved at http://www.ct.gov/dmhas/lib/dmhas/presentations/060807.pdf.

lxxxiv. Corrigan, P. W. (2014). The stigma of disease and disability: Understanding causes and overcoming injustices. Washington, DC: American Psychological Association.

lxxxv. Nemec, P. B., Swarbrick, M., \& Legere, L. (2015). Prejudice and discrimination from mental health service providers. Psychiatric Rehabilitation Journal, 38(2), 203 (p. 204).

lxxxvi. Thorman, S., \& Mendonca K. (2011). Team Building Toolkit, KEYS - Keys to Enhance Your Supervisory Success, University of California, Berkeley. Retrieved at https://hr.berkeley.edu/sites/default/ files/bpm_team_building_toolkit_2019.pdf.

lxxxvii. Delman, J. (2012). Participatory action research and young adults with psychiatric disabilities. Psychiatric Rehabilitation Journal, 35(3), 231.

lxxxviii. Zeldin, S., Christens, B. D., \& Powers, J. L. (2013). The psychology and practice of youth-adult partnership: Bridging generations for youth development and community change. American Journal of Community Psychology, 51(3-4), 385-397.

lxxxix. Bauer, T. N., \& Erdogan, B. (2014). Delineating and reviewing the role of newcomer capital in organizational socialization. Annual Review of Organizational Psychology and Organizational Behavior, 1(1), 439-457.

xc. Jones, N. Peer Involvement and Leadership in Early Intervention in Psychosis Services: From Planning to Peer Support and Evaluation. Retrieved at https://www.nasmhpd.org/sites/default/files/PeerInvolvement-Guidance_Manual_Final.pdf.

xci. Delman, J. (2013). Young Adults Getting Involved: Participatory Action Research \& Transition Age Youth. Retrieved at http://escholarship.umassmed.edu/psych_cmhsr/623/. 


\section{Appendices}
A. Organizational Self Assessment
B. Summary of Federal Anti-discrimination Prohibitions
C. Sample YA Job Description
D. Sample Memo Form
E. PDSA Form for Supervision
F. Standards for Assessing Reasonableness and Undue Hardship
G. Leave and Undue Hardship
H. Job Challenges and Accommodations Worksheet 


\section{Appendix A}

\section{ORGANIZATIONAL SELF-ASSESSMENT}

\begin{tabular}{|c|c|c|c|c|c|}
\hline & $\begin{array}{l}\text { Strongly } \\
\text { Disagree }\end{array}$ & Disagree & $\begin{array}{l}\text { Not sure } \\
\text { /Undecided }\end{array}$ & Agree & $\begin{array}{c}\text { Strongly } \\
\text { Agree }\end{array}$ \\
\hline $\begin{array}{l}\text { 1. Staff understand the purpose and duties of the } \\
\text { peer support worker role. }\end{array}$ & & & & & \\
\hline $\begin{array}{l}\text { 2. Peer support workers in the agency receive } \\
\text { individualized and regular supervision. }\end{array}$ & & & & & \\
\hline $\begin{array}{l}\text { 3. Staff respect and work with the goals and } \\
\text { treatment preferences of young adult clients. }\end{array}$ & & & & & \\
\hline $\begin{array}{l}\text { 4taff understand the unique developmental } \\
\text { experiences of young adults in today's society. }\end{array}$ & & & & & \\
\hline $\begin{array}{l}\text { 5. Staff believe that young adult peer support } \\
\text { workers can be valuable members of the } \\
\text { treatment team. }\end{array}$ & & & & & \\
\hline $\begin{array}{l}\text { 6. Staff will help young adult peer support } \\
\text { workers to conduct their job duties. }\end{array}$ & & & & & \\
\hline $\begin{array}{l}\text { 7. The agency implements policies and trainings } \\
\text { that respect the diversity of different cultures, } \\
\text { (e.g., racial/ethnic, age, gender). }\end{array}$ & & & & & \\
\hline $\begin{array}{l}\text { 8. The agency takes specific steps to address } \\
\text { workplace stigma and discrimination [i.e., } \\
\text { mental illness]. }\end{array}$ & & & & & \\
\hline $\begin{array}{l}\text { 9. The agency provides service recipients with } \\
\text { information about peer support groups and } \\
\text { services. }\end{array}$ & & & & & \\
\hline $\begin{array}{l}\text { 10. Staff orientation and training for all treatment } \\
\text { staff covers the specific responsibilities of } \\
\text { young adult peer support workers. }\end{array}$ & & & & & \\
\hline $\begin{array}{l}\text { 11. Staff understand that young adults with } \\
\text { mental illnesses can be capable of competitive } \\
\text { work with the right supports }\end{array}$ & & & & & \\
\hline $\begin{array}{l}\text { 12. Staff use person-first language in all written } \\
\text { and verbal communication. }\end{array}$ & & & & & \\
\hline $\begin{array}{l}\text { 13. Employees know how to request reasonable } \\
\text { accommodations. }\end{array}$ & & & & & \\
\hline $\begin{array}{l}\text { 14. When an employee requests a reasonable } \\
\text { | accommodation, the supervisor and } \\
\text { employee work together to find the best one. }\end{array}$ & & & & & \\
\hline $\begin{array}{l}\text { 15. Organizational/program leaders communicate } \\
\text { the importance of the young adult peer }\end{array}$ & & & & & \\
\hline
\end{tabular}




\section{Appendix A}

ORGANIZATIONAL SELF-ASSESSMENT

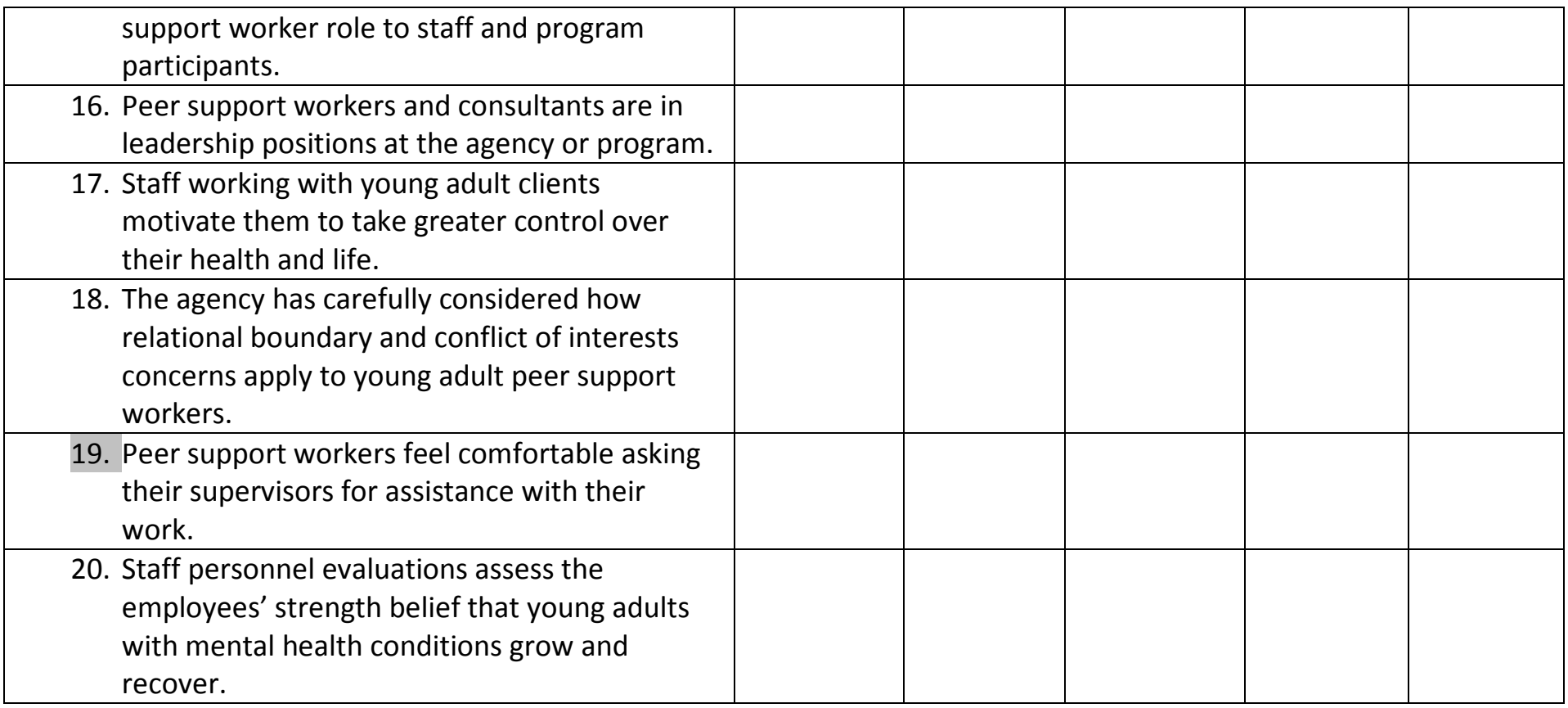




\section{Appendix B}

\section{SUMMARY OF FEDERAL ANTI-DISCRIMINATION PROHIBITIONS}

The Americans with Disabilities Act of 1990 ("ADA") and related state and federal laws generally prohibit organizations from discriminating against qualified people with disabilities who are able to perform the essential job functions, with or without a reasonable accommodation (see appendix E).

Mental health disorders are qualify as disabilities, even if symptoms are controlled by medications or other means. People are also protected under this law when they are "regarded as" having a disability, though they might not actually have one.

Many young people with mental illness also have struggled with substance abuse. Although alcoholism and drug addiction both are disabilities under the ADA, they are treated differently.. An alcoholic is viewed as having a disability.

In general people with a history of drug addiction who not currently using drugs and have been rehabilitated are covered under the ADA. The issues with regarding illegal drug addiction and use are more complex, and addressed briefly in the box at the end of this appendix.

This kind of definition essentially places peer providers within the legally protected class of people with disabilities. That is, peer providers are hired because of their experience of having a mental health condition that impaired functioning of living skills, and are thus at the very least seen having such disability by employers.

Thus, peer provider functions always considered essential are using one's story to assist and inspire clients, providing peer support, and advocating for the clients participation in treatment. The written job description, personnel policies, and the position's professional code of ethics (chapter 1) provide the strongest evidence of essential functions. Essential functions can also include specific duties related to the typical peer role, such as systems navigation and leading wellness classes.

The ADA also prohibits disability-based harassment, offensive conduct that becomes a condition of continued employment, or severe or pervasive conduct that creates what a reasonable person would consider an intimidating or hostile workplace (See EEOC comment). The employer is therefore obligated to encourage reports of harassment and provide an effective complaint or grievance process. The employer is also obligated to investigate reports on other information about harassment.

The ADA essentially mandates equal opportunities in employment for people with disabilities by establishing their rights to reasonable accommodations, assistance or changes to a position or workplace that will enable an employee to do his or her job despite having a disability. We discuss this in greater detail in Chapter 7 and appendix E. 


\section{Illegal drug use and the ADA}

Someone who is addicted to illegal drugs has a disability only if s/he is not currently using illegal drugs. According to the United States Commission on civil rights (http://www.usccr.gov/pubs/ada/ch4.htm):

The ADA provides that any employee or job applicant who is "currently engaging" in the illegal use of drugs is not a "qualified individual with a disability." Therefore, an employee who illegally uses drugs - whether the employee is a casual user or an addict-is not protected by the ADA if the employer acts on the basis of the illegal drug use. As a result, an employer does not violate the ADA by uniformly enforcing its rules prohibiting employees from illegally using drugs.

However, "qualified individuals" under the ADA include those individuals:

- who have been successfully rehabilitated and who are no longer engaged in the illegal use of drugs;

- who are currently participating in a rehabilitation program and are no longer engaging in the illegal use of drugs; and

- who are regarded, erroneously, as illegally using drugs.

A former drug addict may be protected under the ADA because the addiction may be considered a substantially limiting impairment. However, according to the EEOC Technical Assistance Manual on the ADA, a former casual drug user is not protected:

[A] person who casually used drugs illegally in the past, but did not become addicted is not an individual with a disability based on the past drug use. In order for a person to be "substantially limited" because of drug use, s/he must be addicted to the drug. 


\section{Appendix C}

\section{YOUNG ADULT PEER SUPPORT SPECIALIST SAMPLE JOB DESCRIPTION}

Job Title: Young Adult (YA) Peer Support Specialist

Status: Full-time

Work Site:

Reporting Structure: Reports to Transition-Age Youth Team Leader

Purpose of Job: The YA Peer Support Specialist will: (1) serve as an integral part of a multidisciplinary team through using his or her personal lived experience to connect with, validate, inspire, and provide support to transition-age youth diagnosed with serious mental health conditions in reaching their goals and (2) increase community awareness of mental health challenges among transition-age youth and what helps young people to cope with these challenges.

\section{Duties \& Responsibilities:}

- Assist TAY clients in developing self-advocacy and negotiating skills. Serve as a role model to TAY clients in communication, health and wellness, and conflict resolution.

- Share lived-experience story, as appropriate, to establish credibility and trust with TAY clients and their families.

- Partner with the TAY client and the team to identify the TAY's strengths, needs, and goals. Support TAY in developing specific, practical steps to reach their goals with the team. Support TAY in achieving goals.

- Collaborate on all support activities with the team when a TAY client indicates need for and acceptance of YA Peer Support Specialist involvement.

- Assist the team in partnering with TAY clients to support engagement in services, articulate preferences and concerns, and identify people within their support network and new resources that will support their personal goal attainment.

- Facilitate individual and group meetings with TAY clients that promote sharing, learning, and growth. These meetings are designed to meet the needs of the TAY in a particular context - thus they may be manual led, unstructured topical groups, skill-based groups, or social events.

- Assist team in developing language that accurately reflects the preferences of TAY who are 
considering and/or engaged in services.

- Participate in individual and group supervision, multidisciplinary consultation meetings, team meetings, agency required training, and external trainings as applicable to position. Trainings may include: Motivational Interviewing, Achieve My Plan! (AMP), Wellness Management and Recovery Action Plan (WRAP), and/or Gathering Inspiring Future Talent (GIFT).

- Participate in discussions and learning opportunities that enhance skill set and connect the YA Peer Support Specialist to peers in similar roles at different agencies.

- Engage in community outreach and education. Assist team with championing initiatives to increase TAY client engagement in services. Share lived-experience as part of team outreach and education efforts.

- Document contacts/ activities per requirements of Performance Specifications for Therapeutic Mentoring.

\section{Job Specific Qualifications, Experience, Education \& Professional Attributes:}

- Possesses lived experience in effectively coping with a serious mental health condition and a capacity to share his or her recovery story in a way that engages, inspires, and validates the experiences of TAY clients.

- Ability to effectively partner with TAY clients and team members to support TAY engagement and outcomes; able to work collaboratively with a team.

- Possesses a desire to ensure that TAY clients are heard; verbally communicates and advocates in a respectful manner.

- Has made sufficient progress with education and employment in order to prepare them to successfully engage TAY clients and support goal attainment.

- Openness to working with a diverse TAY clientele in a sensitive and competent manner.

- Ability to effectively share information about services, resources, and TAY client role and responsibilities to TAY clients, their self-identified support network, and caregivers.

- Ability to teach and role model communication and self-advocacy skills in a variety of settings (e.g., with informal social network, providers, organizations, courts, etc.).

- Ability and willingness to participate in and contribute to team activities including trainings, supervision, community and statewide meetings.

- Ability to complete service documentation using agency software in a timely manner and possesses a capacity to communicate effectively and professionally through email and phone.

- Ability to travel with and/or transport persons served and travel to meetings (via public transit or car). Must possess a current/valid driver's license, have a valid driving record with a clean driving history and a reliable automobile with proof of auto insurance or demonstrate competency in use of public transportation which can be modeled for young adults being served. 


\section{Appendix D}

\section{[ORGANIZATION]}

To: $\quad$ [Recipient names]

From:

CC: [Recipient names]

Date: [Pick the date]

Re: $\quad$ [Subject]

Comments: [Your comments]

\section{Sample Structure}

- Introduction (if longer)

- The question(s) you were asked to look into

- In what ways did you look into it, what sources did you yse

- What you learned

- Additional thoughts

- Conclusions 
Appendix E

\section{PDSA Cycle Template}

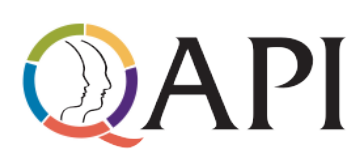

Directions: Use this Plan-Do-Study-Act (PDSA) tool to plan and document your progress with tests of change conducted as part of chartered performance improvement projects (PIPs). While the charter will have clearly established the goals, scope, timing, milestones, and team roles and responsibilities for a project, the PIP team asked to carry out the project will need to determine how to complete the work. This tool should be completed by the project leader/manager/coordinator with review and input by the project team. Answer the first two questions below for your PIP. Then as you plan to test changes to meet your aim, answer question 3 below and plan, conduct, and document your PDSA cycles. Remember that a PIP will usually involve multiple PDSA cycles in order to achieve your aim. Use as many forms as you need to track your PDSA cycles.

Model for Improvement: Three questions for improvement

1. What are we trying to accomplish (aim)?

State your aim (review your PIP charter - and include your bold aim that will improve resident health outcomes and quality of care)

2. How will we know that change is an improvement (measures)?

Describe the measureable outcome(s) you want to see

3. What change can we make that will result in an improvement?

Define the processes currently in place; use process mapping or flow charting

Identify opportunities for improvement that exist (look for causes of problems that have occurred see Guidance for Performing Root Cause Analysis with Performance Improvement Projects; or identify potential problems before they occur - see Guidance for Performing Failure Mode Effects Analysis with Performance Improvement Projects) (see root cause analysis tool):

- Points where breakdowns occur

- "Work-a-rounds" that have been developed

- Variation that occurs

- Duplicate or unnecessary steps

Decide what you will change in the process; determine your intervention based on your analysis

- Identify better ways to do things that address the root causes of the problem

- Learn what has worked at other organizations (copy)

- Review the best available evidence for what works (literature, studies, experts, guidelines)

- Remember that solution doesn't have to be perfect the first time 
What changes are we going to make based on our findings?

What were the results?
What exactly are we going to do?
When and how did we do it?

\begin{tabular}{|c|c|c|}
\hline Plan & $\begin{array}{l}\text { What change are you testing with } \\
\text { the PDSA cycle(s)? } \\
\text { What do you predict will happen and } \\
\text { why? } \\
\text { Who will be involved in this PDSA? } \\
\text { (e.g., one staff member or resident, } \\
\text { one shift?). Whenever feasible, it } \\
\text { will be helpful to involve direct care } \\
\text { staff. } \\
\text { Plan a small test of change. } \\
\text { How long will the change take to } \\
\text { implement? } \\
\text { What resources will they need? } \\
\text { What data need to be collected? }\end{array}$ & $\begin{array}{l}\text { List your action steps along with person(s) responsible and } \\
\text { time line. }\end{array}$ \\
\hline Do & $\begin{array}{l}\text { Carry out the test on a small scale. } \\
\text { Document observations, including } \\
\text { any problems and unexpected } \\
\text { findings. } \\
\text { Collect data you identified as } \\
\text { needed during the "plan" stage. }\end{array}$ & Describe what actually happened when you ran the test. \\
\hline
\end{tabular}


Study and analyze the data.

Describe the measured results and how they compared to the

Determine if the change resulted in predictions.

the expected outcome.

Were there implementation

lessons?

Summarize what was learned. Look for: unintended consequences, surprises, successes, failures.

Act

Describe what modifications to the plan will be made for the

Based on what was learned from the test: next cycle from what you learned.

Adapt - modify the changes and repeat PDSA cycle.

Adopt - consider expanding the changes in your organization to additional residents, staff, and units. Abandon - change your approach and repeat PDSA cycle. 


\section{Appendix F}

\section{STANDARDS FOR ASSESSING REASONABLENESS AND UNDUE HARDSHIP}

\section{1) Key Reasonableness Questions}

- Will the accommodation help the employee perform the essential job functions?

- gNhat is the likelihood that the accommodation will successfully address the difficulty?

- $\square$ What has worked here in the past for people with disabilities in similar situations?

- $\square$ What has worked in the past for this specific employee in this or in previous jobs?

- $\square$ What are the recommendations of health care professionals?

- $\square$ What is the preference of the employee?

- $\llbracket \mathrm{C}$ Can it be implemented in a reasonable amount of time?

\section{2) Undue Hardship Considerations}

- $\square$ The nature and net cost of the accommodation;

- $\square$ The type of the employer's operation, including composition and structure of workforce; (companies with vast diversity of job types are more likely to face undue hardships);

- The impact of the accommodation on the operation of the facility/program (such as the impact on the ability of other employees to perform their duties);

- The size of the organization), including:

o number of employees,

o number and type of facilities

o size of budget or available assets

(Smaller companies, with fewer employees and programs/facilities are more likely to face undue hardships with a proposed accommodation)

See also- https://askjan.org/ 


\section{3) Undue Hardship and the young adult peer}

- Accommodation costs are usually indirect:

o YA peer supervisor providing additional supervision;

o When a peer reduces weekly hours and takes leave, other roles on the team may need to be restructured.

- Direct financial costs are not common; they are usually payments for external training or support (e.g., class fees or job coaches) and for replacement staff when the peer takes a leave of absence;

- The YA peer provider can be difficult to replace because of the unique qualities and skills necessary for the role and the limited number of YA peers on staff. Thus "leave" for a YA peer is more likely to present undue hardship issues than for other employees, at least from an operational perspective.

- It is the company's obligation to make every effort to cover the YA peer's hours. 


\section{Appendix G}

\section{LEAVE AND UNDUE HARDSHIP: THE BASICS AND PRACTICAL APPLICATIONS}

Many supervisors struggle to decide at what point an approved leave becomes an undue hardship for the organization. The basic issue for supervisors is whether the provider can deliver services according to professional and contractual expectations without making major financial expenditures. Leave of key staff for a substantial length of time can interrupt clinical operations, and there may be direct costs of bringing in temporary staff. In addition, peer responsibilities are difficult to replace (when few other staff have the peer's special qualifications). Ultimately, the key questions the supervisor must consider are:

1. Are services being delivered according to requisite provider and peer support service standards?

2. Can another employee handle those responsibilities effectively and for how long?

3. Can a "temp" peer be hired or contracted with for those responsibilities?

If the employee's leave request is "reasonable", the employer must make every effort to support the continuation of the peer role, with the understanding that some staff and clients may be temporarily inconvenienced. As the leave becomes longer it may start to feel less reasonable to the employer due to questions about the employee's return and the stress of holding a job open and not hiring a new person. Thus, employers should make an effort to document the impact of employees' absences on operations.

Additional important guidelines on leave as a Reasonable Accommodation (RA) include:

- In seeking leave as a reasonable accommodation, the employee need not show that the leave is certain (or even likely) to be successful in improving job performance, only that it could plausibly enable the employee to return and perform his/her job.

- For ADA purposes, the employer has the right to consider as most reasonable an alternative to leave.

- Employers are encouraged to request an approximate anticipated date of return.

- An employee may revise specific or estimated work return dates when it is medically justified, return to work remains feasible, and, it would not cause an undue hardship.

- Employers should through some fashion (e.g., phone, text) stay in touch with the employee and offer emotional support and encouragement.

- "Return to work planning" is a good idea, often supported through an Employee Assistance Program (see chapter 4).

- An employer can inform an employee on leave that continued absence would be unreasonable or an undue hardship as of a certain date, providing the reason(s), for this determination while identifying other possible accommodations that would work (e.g., return part-time, lightened job responsibilities).

For more information, the Job Accommodation Network (JAN) has general guidelines for "leave" at- https://askjan.org/topics/leave.cfm. 


\section{Appendix H}

Job Challenges and Accommodations Worksheet https://workplaceaccommodations.net/ 